\title{
Coupled-Cluster Techniques for Computational Chemistry: the CFOUR Program Package
}

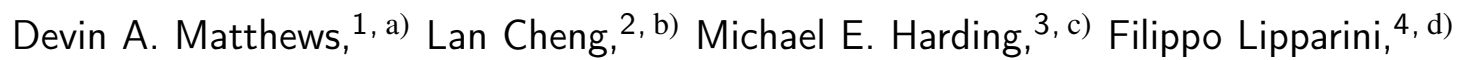
Stella Stopkowicz, ${ }^{5, \text { e) }}$ Thomas-C. Jagau, ${ }^{6, \text { f) }}$ Péter G. Szalay, ${ }^{7, g}$ ) Jürgen Gauss, ${ }^{5, h)}$ and John F. Stanton ${ }^{8, \text { i) }}$

${ }^{1)}$ Department of Chemistry, Southern Methodist University, Dallas, TX 75275

${ }^{2)}$ Department of Chemistry, Johns Hopkins University, Baltimore, MD 21218

${ }^{3)}$ Institut für Physikalische Chemie, Karlsruher Institut für Technologie (KIT), Kaiserstr. 12, D-76131 Karlsruhe, Germany

${ }^{4)}$ Dipartimento di Chimica e Chimica Industriale, Università di Pisa, Via G. Moruzzi 13, I-56124 Pisa, Italy

${ }^{5)}$ Department Chemie, Johannes Gutenberg-Universität Mainz, Duesbergweg 10-14, D-55128 Mainz, Germany

${ }^{6)}$ Department of Chemistry, University of Munich (LMU), Butenandtstr. 5-13, D-81377 Munich, Germany

7) ELTE Eötvös Loránd University, Institute of Chemistry, Pázmány Péter sétány 1/A, H-1117 Budapest, Hungary

${ }^{8)}$ Quantum Theory Project, Departments of Chemistry and Physics, University of Florida, Gainesville, FL 32611

(Dated: 8 April 2020) 
An up-to-date overview of the CFOUR program system is given. After providing a brief outline of the evolution of the program since its inception in 1989, a comprehensive presentation is given of its well-known capabilities for high-level coupled-cluster theory and its application to molecular properties. Subsequent to this generally well-known background information, much of the remaining content focuses on lesser-known capabilities of CFOUR, most of which have become available to the public only recently or will become available in the near future. Each of these new features is illustrated by a representative example, with additional discussion targeted to educating users as to classes of applications that are now enabled by these capabilities. Finally, some speculation about future directions is given, and the mode of distribution and support for CFOUR are outlined in the appendix.

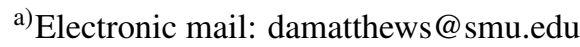

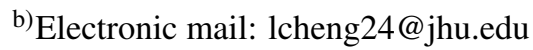

${ }^{c}$ Electronic mail: michael.harding@kit.edu

${ }^{\mathrm{d})}$ Electronic mail: filippo.lipparini@ unipi.it

e)Electronic mail: stella.stopkowicz@uni-mainz.de

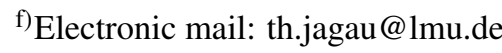

g)Electronic mail: szalay@chem.elte.hu

h)Electronic mail: gauss@uni-mainz.de

i)Electronic mail: johnstanton@ufl.edu
} 


\section{INTRODUCTION}

The origin of the CFOUR (Coupled-Cluster techniques for Computational Chemistry) program package $^{1}$ is deeply connected with the story of several young scientists crossing paths at an early stage of their careers in Rodney J. Bartlett's group at the Quantum Theory Project at the University of Florida in Gainesville, near the dawn of the 1990s. After attending the inaugural Molecular Quantum Mechanics (MQM) meeting in honor of John A. Pople in Athens, GA, in October 1989, John F. Stanton was inspired by the rapid development around the world in high-accuracy quantum chemical methods, and especially by the rapid progress that was being made in their application to interesting and "real" chemical problems. Educated in the Bartlett group, he had been fully convinced of the power of high-level many-body methods, and was determined to develop a new set of programs to bring these approaches to bear on meaningful chemical applications. Upon his return to Gainesville, Stanton started a project that has now lasted more than three decades, which has led to what is now known as CFOUR. By the end of 1989, he had written interfaces to the self-consistent field (SCF) and integral packages used in the Bartlett group - the ACES (Advanced Concepts in Electronic Structure) program system. ${ }^{2}$ In 1990, Jürgen Gauss arrived in Gainesville for a postdoc in the Bartlett group, which fueled the development of the project. Together, Stanton and Gauss wrote many-body perturbation theory (MBPT) ${ }^{3}$ and coupled-cluster $(\mathrm{CC})^{4}$ codes - the latter through CC with singles and doubles (CCSD) - that included analytic gradients ${ }^{5}$ as well as the exploitation of molecular point-group symmetry ( $D_{2 h}$ and subgroups). ${ }^{6}$

John D. Watts, another postdoc in the Bartlett group at that time, contributed code for triple excitations, and Walter J. Lauderdale, a graduate student, wrote a new SCF and integral transformation program. Together with atomic orbital (AO) integrals coming from the MOLECULE package $^{7}$ of Jan Almlöf (one- and two-electron integrals; the code had recently been extensively modified for performance on vector processors by Peter R. Taylor), the VPROPS package ${ }^{8}$ (dipole and other one-electron property integrals, which can trace its lineage back to the POLYATOM package $^{9}$ ), and integral derivatives coming from the ABACUS package ${ }^{10}$ of Trygve Helgaker $e t$ $a l$. , the main core of what was to become CFOUR had already emerged. Apart from AO integral and integral derivative evaluation, all other code was completely new; nothing associated with Hamiltonian construction, MBPT and CC energy and density evaluation was taken from another source; indeed even input parsing and general processing of output (vibrational frequencies, for example) was written from scratch. With this nucleus, a number of chemical applications ${ }^{11-13}$ 
were done at the dawn of the 1990s, and a first paper ${ }^{14}$ describing the code - called ACES II at that time - was published in 1992.

Following the move of the main developers: Stanton to Austin, TX, and Gauss to Karlsruhe (Germany) and later Mainz (Germany) the main development centers of ACES II migrated from the original Gainesville location, taking their exposure to many-body methods with them. This eventually resulted in a bifurcation of ACES II, comprising the Mainz-Austin-Budapest (MAB) version - the Budapest center (Hungary) involving Péter G. Szalay as another main author. In Gainesville, this was followed by a complete rewrite of the overall package devised to target emergent parallel computers. This is known now as the ACES III package. ${ }^{15}$ Finally, in 2008, the Mainz-Austin-Budapest version of ACES II, by now containing many new features and enhanced computational sophistication, was renamed as CFOUR. ${ }^{16}$

Since its beginnings, CFOUR has specialized in high-accuracy quantum chemical methods, targeting applications in the field of thermodynamic, spectroscopic and kinetic phenomena of small- to medium-sized molecular systems. While some of its nearly thirty-year-old primordial core remains in the current version, much has also changed since its inception. Incremental algorithmic improvements have been made to existing capabilities, and new methodology has been continuously added to the package by developers throughout the world. Some of the capabilities included today (together with their first appearance in CFOUR) are: NMR chemical shifts ranging from second-order MBPT through CCSD(T) (1990s); ${ }^{17-23}$ equation-of-motion coupled cluster methods for electronic excited and ionized states; ${ }^{24-29}$ analytic second derivatives for MBPT and CC through CCSDT (1990s); ${ }^{23,30-33}$ automated evaluation of anharmonic (quartic) force fields and computation of associated rovibrational spectroscopic constants (1990s); ${ }^{34,35}$ new open-shell CC methods (1990s); ${ }^{36,37}$ properties associated with high-resolution spectroscopy such as spin-rotation tensors (1990s and 2000s); ${ }^{35,38-41}$ arbitrarily high-order CC gradients and second derivatives (as interfaced to the MRCC package ${ }^{42,43}$ of Mihály Kállay, 2000s); ${ }^{44-47}$ diagonal Born-Oppenheimer corrections (2000s) $;{ }^{48,49}$ couplings between quasidiabatic states (2010s); ${ }^{50,51}$ relativistic quantum chemical methods (2010s); ${ }^{52-60}$ multireference CC methods (2010s); ${ }^{61}$ highly efficient code for high-accuracy (post-CCSD(T)) methods (2010s); ${ }^{62}$ and many more.

Following the work of the original team, and beginning their careers in the groups of the main authors, many more young scientists actively contributed to CFOUR. The primary authors of CFOUR now include Lan Cheng, who has contributed extensively with relativistic quantum chemical methods ${ }^{56,58-60}$ for both energy and property calculations; Devin A. Matthews, who has 
written a new and very fast coupled-cluster module (xncc) ${ }^{62}$ for CFOUR and contributed significantly to some of the spectroscopic extensions of CFOUR; ${ }^{63,64}$ and Michael E. Harding, who has been in charge of many issues related with code infrastructure, parallelization, ${ }^{65,66}$ and general organization.

An accurate characterization of CFOUR is that it is a program system with many capabilities for the highly accurate calculation of parameters that play a role in diverse areas of chemical physics. Largely through methods based on coupled-cluster theory, ${ }^{4}$ one can calculate potential energy surfaces, couplings between electronic states, a vast number of one- and two-electron properties that play a role in various branches of molecular spectroscopy, relativistic corrections to electronic structure, and generally obtain information that can be extracted from accurate electronic wavefunctions and their response to external perturbations. Beyond this, there are auxiliary tools that make use of this fundamental information. For example, vibrational perturbation theory ${ }^{67}$ can be used to obtain accurate positions for the fundamental vibrational levels of semirigid polyatomic molecules (using the efficiently-calculated anharmonic force field); information can be extracted to construct vibronic Hamiltonians in a diabatic representation; extrapolation to the basis set limit can be done in an automated fashion; ${ }^{68}$ molecular structures can be fitted to rotational constants, ${ }^{35}$ (both the raw experimental data and the equilibrium constants corrected (by CFOUR calculations) for the effects of vibration-rotation interaction; ${ }^{34,67}$ etc.

The capabilities of CFOUR can be also used in conjunction with features of other computational chemistry programs (e.g., MRCC ${ }^{42,43}$ GIMIC, ${ }^{69}$ NEWTON-X, ${ }^{70-72}$ and GECCO ${ }^{73,74}$ ) to which CFOUR has been interfaced.

While providing powerful tools for the quantum chemical study of small- to medium-sized molecules, CFOUR does not have a great deal to offer in the area of large molecules. Developments in CFOUR have focused on many-body treatments of electron correlation, and the methods of density functional theory are completely absent from its repertoire. The coupled-cluster methods available in CFOUR are mainly single-reference methods, meaning that calculations are built upon a single Slater determinant that is usually (but need not be) composed of orbitals associated with the Hartree-Fock self-consistent field (HF-SCF) solution. While some multireference effects can certainly be treated within the framework of equation-of-motion coupled-cluster (EOM-CC) methods ${ }^{75}$ - this area represents a decided strength of CFOUR - more traditional methods based on multiconfigurational zeroth-order wavefunctions are needed to describe phenomena associated with bond-breaking, to construct (semi-)global potential energy surfaces, and even to treat certain 
classes of transition states. While some of these limitations described above have been addressed by implementing multireference variants of $\mathrm{CC}$ theory ${ }^{61,76}$ and incorporating a rigorous secondorder complete-active space SCF (CASSCF) scheme ${ }^{77}$ in CFOUR, the currently available version of the program exclusively offers single-reference treatments of the correlation problem.

The remainder of this paper elaborates on the strengths and capabilities of the CFOUR program system. The next section summarizes the "core features" of CFOUR, specifically its treatments of the non-relativistic electronic Schrödinger equation based on CC and MBPT methods, and its capabilities for calculating properties within these approximations using analytic derivative techniques. Many users of CFOUR are likely to be familiar with these capabilities, and the section largely serves to document these features with some remarks about the current status of implementations. We continue with a short section about practical aspects such as input and use of CFOUR. After that, a section is included to describe new developments that are present in CFOUR, either in the current version (V2.1) or versions likely to come in the near future. After the discussion of the present state of the CFOUR project, we proceed with some remarks about the general longterm perspective on the future of CFOUR and close by describing the method by which the code is distributed.

\section{ESTABLISHED FEATURES}

\section{A. Treatments of electron correlation}

The available treatments of electron correlation in CFOUR are based on many-body perturbation theory (MBPT, also known as Møller-Plesset (MP) perturbation theory) ${ }^{3,78}$ and coupledcluster (CC) theory ${ }^{4,79,80}$ and collectively referred to as single-reference methods, as their description of electron correlation starts from a single Slater determinant.

$\mathrm{CC}$ theory was originally formulated for the quantum-chemical treatment of nuclear matter. ${ }^{81,82}$ After its introduction into electronic structure theory by Čížek ${ }^{83,84}$ in 1966, it developed to one of the most powerful schemes quantum chemistry nowadays has to offer for the electron-correlation treatment and for high-accuracy computations. The success of CC theory is probably best illustrated by the fact that the $\operatorname{CCSD}(\mathrm{T})$ method, ${ }^{85}$ to be described in detail below, often is referred to as the "gold standard" in quantum chemistry. 
$\mathrm{CC}$ theory uses an exponential ansatz for the wavefunction

$$
|\psi\rangle=\exp (T)|0\rangle
$$

where $|0\rangle$ denotes the reference determinant (often, but not necessarily chosen as the HF state) and $T$ the cluster operator which is an excitation operator and consists of the weighted sum of all excitations

$$
T=T_{1}+T_{2}+\ldots T_{N}
$$

The sum in Eq. (2) runs up to $T_{N}$ with $N$ as the number of electrons. $T_{1}, T_{2}, \ldots$ denote the weighted sums of single, double, ... excitations with the unknown parameters given by the weighting coefficients that are usually referred to as amplitudes. The chosen wavefunction ansatz in Eq. (1) has significant advantages over the corresponding linear choice in configuration-interaction $(\mathrm{CI})$ theory, as it ensures size-consistency ${ }^{86} /$ size-extensivity $^{87}$ of the electron-correlation treatment even within a truncated scheme that does not include all excitations. CC theory therefore is, by construction, a size extensive approach.

Because of the exponential ansatz, the $\mathrm{CC}$ wavefunction is typically not determined via the variational principle. Instead, one uses a projection approach in which the CC wavefunction is inserted into the electronic Schrödinger equation, the latter is then multiplied from the left with $\exp (-T)$, and an expression for the energy is obtained by projection onto the reference determinant

$$
E=\langle 0|\exp (-T) H \exp (T)| 0\rangle
$$

and nonlinear equations for the amplitudes are obtained by projection onto the excited determinants

$$
0=\left\langle\Phi_{P}|\exp (-T) H \exp (T)| 0\right\rangle .
$$

In Eqs. (3) and (4), $H$ denotes the usual molecular Hamiltonian and $\Phi_{P}$ a determinant from the manifold of excited determinants. The nonlinear amplitude equations, Eq. (4), consequently need to be solved for all possible $\Phi_{P}$.

Without any truncation, $\mathrm{CC}$ theory is equivalent to, though more involved than, full configuration interaction (FCI) and hence in that form not particularly useful. CC theory demonstrates its advantages only when used with a truncated cluster operator. The usual choices are here $T=T_{2}$ (CC doubles, CCD), ${ }^{88-90} T=T_{1}+T_{2}$ (CC singles and doubles, CCSD), ${ }^{91} T=T_{1}+T_{2}+T_{3}$ 
(CC singles, doubles, triples, CCSDT), ${ }^{92,93}$ and $T=T_{1}+T_{2}+T_{3}+T_{4}$ (CC singles, doubles, triples, quadruples, CCSDTQ), ${ }^{44,94,95}$ etc. While initially the implementation of CC methods was quite cumbersome, ${ }^{89-91}$ the use of intermediates together with a rewrite of the equations in terms of matrix-vector products has enabled more straightforward access to CC methods ${ }^{6,95,96}$ and forms also the basis of the CCSD implementations in CFOUR which is described in detail in Ref. 6. CFOUR also offers the possibility to perform CCSDT ${ }^{92,93,97}$ as well as CCSDTQ calculations. ${ }^{44,62,94,95}$ In addition, via an interface to the MRCC code, ${ }^{42} \mathrm{CC}$ computations with arbitrary excitations are possible. ${ }^{44}$

While CCSD is for many applications not accurate enough and CCSDT with an $M^{8}$ scaling ( $M$ denotes here the system size, which is assumed proportional to both the number of occupied and virtual orbitals) too expensive, approximate $\mathrm{CC}$ methods have been developed in which not only the cluster operator is truncated but (expensive) terms in the CC equations are also neglected. This leads in the case of triple excitations in a straightforward manner to the CCSDT- $n$ methods. ${ }^{98,99}$ The key idea is here to (a) skip the $M^{8}$ terms and (b) avoid storage of the triples amplitudes. The selection of the terms in the triples equations is then based on perturbation theory and leads to CCSDT- $1 \mathrm{a},{ }^{98}$ CCSDT- $1 \mathrm{~b},{ }^{98}$ CCSDT- $2,{ }^{99}$ and CCSDT- $3 .{ }^{99}$ Somewhat related to CCSDT- $1 \mathrm{~b}$ is the $\mathrm{CC} 3$ model $^{100}$ which has been introduced by the Aarhus group in the context of CC response theory. ${ }^{101}$ All these models (CCSDT- $n$ with $n=1-3$ and CC3) scale with $M^{7}$ and do not require storage of triple excitation amplitudes. The $\mathrm{CC} 3$ model furthermore is part of an alternative hierarchy of CC methods: CC2, CCSD, CC3, CCSDT, CC4, ... in which CC2 $2^{102}$ is the simplest choice and a cheap approximation to CCSD with a $M^{5}$ scaling and no need to store double excitation amplitudes. In this context one should also mention the quadratic CI singles and doubles (QCISD) scheme ${ }^{103}$ by Pople et al., which was introduced as a simpler alternative to CCSD. However, as there are nowadays no difficulties to implement CCSD, QCISD now plays only a minor role.

The CCSDT- $n$ and CC3 models are significantly more efficient in computational terms than the full CCSDT model, but they are for many applications still not affordable due to the need to consider triple excitations in each iteration. This issue can be ameliorated by just considering a perturbative correction for triple excitations on top of a CCSD computation. Starting with early ideas based on corrections taken from fourth order MBPT or MP theory, ${ }^{99,104}$ ultimately led to the development of the (T) correction which involves the fourth-order correction due to triple excitations, ${ }^{85,105}$ though computed with the converged CCSD amplitudes, together with one fifthorder correction, namely the one that couples singles and triples. Justifications for this choice 
have been for example given in Refs. 106 and 107. Similar ideas as in the case of CCSD(T) for triple excitations can be also pursued for the perturbative treatment of quadruple excitations, which leads to the CCSDT(Q) approach. ${ }^{108,109}$ More elaborate triple and quadruple corrections ${ }^{110}$ (referred to as $\Lambda$-CCSD $(\mathrm{T})^{111}$ and $\Lambda$-CCSDT $(\mathrm{Q})^{109}$ ) can be obtained by using the solution of the $\Lambda$ equations in addition to those of the amplitude equations for the evaluation of the perturbative corrections. ${ }^{107,109,111,112}$

Considering the treatment of closed- and open-shell systems, CFOUR offers spin-adapted treatments for closed-shell systems and open-shell treatments based on UHF and ROHF reference determinants. ${ }^{12}$ The UHF-CC treatment is a straightforward spin-orbital based approach, though with spin integration, while ROHF-CC ${ }^{113}$ formally classifies as a non-HF CC approach as the occupied-virtual block of the Fock matrix in the spin-orbital basis does not vanish. However, this only requires the trivial inclusion of off-diagonal elements of the Fock-matrix in the $\mathrm{CC}$ equations within a standard CC treatment, but some thought is required to formulate appropriate perturbative corrections. ${ }^{14,115}$ The latter are most efficiently implemented using so-called semicanonical orbitals. ${ }^{116} \mathrm{CC}$ calculations can also be carried out using the quasi RHF (QRHF) determinant ${ }^{113}$ as reference (here the orbitals for the reference determinant are obtained in a RHF calculation with a different number of electrons). Further options involve Brueckner CC (B-CC) ${ }^{117,118}$ and orbital-optimized CC calculations. ${ }^{119}$ In both cases, the orbitals are determined in the presence of electron correlation which, though more expensive, sometimes turn out to be more efficient.

MBPT can be derived using perturbative techniques together with the Møller-Plesset partitioning ${ }^{120}$ of the electronic Hamiltonian. Alternatively, expressions for the various orders of MBPT can be obtained through perturbative expansions of the CC energy expression as well of the CC amplitude equations. Second-order MBPT, known as MBPT(2) or MP2, has evolved over the years to the standard scheme for a first (and not particularly accurate) description of electron correlation at low cost (the formal scaling is only of the order of $M^{5}$ ) for otherwise rather well behaved systems. Higher-order MBPT schemes (up to sixth order) have also been formulated and implemented, ${ }^{86,121-125}$ but are only rarely used. The reasons are the now well established convergence problems of $\mathrm{MBPT}^{126,127}$ as well the availability of the more robust CC methods. Nevertheless, MBPT(3) (equivalent to MP3) and MBPT(4) (equivalent to MP4) are accessible through CFOUR. MBPT(5) and MBPT(6) are only available in specialized codes, ${ }^{123-125}$ while even higher order MBPT corrections so far can only be extracted from a perturbative dissection of FCI. ${ }^{128,129}$ 
MBPT is rather straightforward to formulate for restricted and unrestricted HF (RHF and UHF) reference functions. However, after some experimentation, ${ }^{130-132}$ a satisfactory formulation of MBPT for restricted open-shell HF (ROHF) reference functions has been suggested. ${ }^{116,133,134}$ The perturbed Hamiltonian contains here also the virtual-occupied blocks of the Fock matrices in a spin-orbital formulation and a non-iterative treatment is possible when semicanonical orbitals are used. ${ }^{116}$

Table I summarizes the CC and MBPT/MP methods that are currently available in the current public version (V2.1) of CFOUR together with information about the possible choices for the reference determinants.

\section{B. Analytic derivatives for the computation of molecular properties}

A particular strength of CFOUR is its ability to provide analytic derivatives of the energy and thus easy access to molecular properties for most implemented quantum-chemical methods. Analytic derivative techniques ${ }^{136,137}$ play an important role for the computation of molecular geometries, as only analytically evaluated forces render geometry optimizations routinely doable. CFOUR offers geometrical derivatives ${ }^{5,32,45,114,138-141}$ for most of the implemented CC and MBPT methods and thus allows the routine determination of equilibrium geometries (preferably via the BFGS scheme ${ }^{142}$ ) but also of transition-state geometries using methods based on eigenvector following. ${ }^{143}$

In CC theory, analytic gradients have been formulated ${ }^{144,145}$ and implemented ${ }^{144}$ rather late. The main reason is the non-variational character of the standard CC approaches. Straightforward differentiation of the CC energy expression, Eq. (3), with respect to a perturbation $x$ thus leads to an expression that involves the derivatives of the cluster operator

$$
\begin{aligned}
\frac{d E}{d x}=\langle 0| & \exp (-T) \frac{d H}{d x} \exp (T)|0\rangle \\
& +\left\langle 0\left|\left[\exp (-T) H \exp (T), \frac{d T}{d x}\right]\right| 0\right\rangle .
\end{aligned}
$$

Evaluation of gradients based on this expression would offer little advantage over a finitedifference approach. However, based on the interchange theorem of perturbation theory, ${ }^{146}$ the derivative expression can be reformulated such that the derivatives of the cluster operator $T$ are no longer needed. This has been shown by Adamowicz and Bartlett, ${ }^{147}$ thereby introducing the perturbation-independent $\Lambda$ equations, and used by Scheiner et al. ${ }^{144}$ for their implementation of 
TABLE I. CC and MBPT/MP methods available in the CFOUR program package. ${ }^{\mathrm{a}}$ A single $\mathrm{x}$ designates that only energy evaluations are possible, while xx indicates that both energy and gradients can be calculated and $\mathrm{xxx}$ indicates that analytic second derivatives are available.

\begin{tabular}{|c|c|c|c|c|}
\hline method & RHF & UHF & ROHF & remarks \\
\hline $\mathrm{HF}$ & $\mathrm{xxx}$ & $\mathrm{xxx}$ & $\mathrm{xxx}$ & \\
\hline MBPT(2)/MP2 & $\mathrm{xxx}$ & $\mathrm{xxx}$ & $\mathrm{xx}$ & \\
\hline MBPT(3)/MP3 & $\mathrm{xxx}$ & $\mathrm{xxx}$ & $\mathrm{xx}$ & \\
\hline SDQ-MBPT(4)/SDQ-MP4 & $\mathrm{x}$ & $\mathrm{x}$ & $\mathrm{x}$ & \\
\hline MBPT(4)/MP4 & $\mathrm{xxx}$ & $\mathrm{xxx}$ & $\mathrm{x}$ & \\
\hline CCD & $\mathrm{xxx}$ & $\mathrm{xxx}$ & & \\
\hline CCSD & $\mathrm{xxx}$ & $\mathrm{xxx}$ & $\mathrm{x}$ & also Brueckner, orbital-optimized CCSD, QRHF-CCSD \\
\hline CCSDT & $\mathrm{xxx}$ & $\mathrm{x}$ & $\mathrm{x}$ & \\
\hline CCSDTQ & $\mathrm{xx}$ & & & \\
\hline CCSDT- $n, n=1 \mathrm{a}, 1 \mathrm{~b}, 2,3$ & $\mathrm{xxx}$ & $\mathrm{xxx}$ & & \\
\hline CCSDTQ- $n, n=1 \mathrm{a}, 1 \mathrm{~b}, 3$ & $\mathrm{xx}$ & & & \\
\hline $\mathrm{CC} 2$ & $\mathrm{xxx}$ & $\mathrm{xxx}$ & & inefficient code, $M^{6}$ scaling \\
\hline $\mathrm{CC} 3$ & $\mathrm{xxx}$ & $\mathrm{xxx}$ & & \\
\hline $\mathrm{CC} 4$ & $\mathrm{xx}$ & & & \\
\hline $\mathrm{CCSD}+\mathrm{T}(\mathrm{CCSD})$ & $\mathrm{x}$ & $\mathrm{x}$ & & \\
\hline $\operatorname{CCSD}(\mathrm{T})$ & $\mathrm{xxx}$ & $\mathrm{xxx}$ & $\mathrm{xx}$ & \\
\hline$\Lambda$-CCSD(T) & $\mathrm{xx}$ & $\mathrm{x}$ & & \\
\hline $\mathrm{CCSD}+\mathrm{TQ}(\mathrm{CCSD})$ & $\mathrm{x}$ & & & \\
\hline $\mathrm{CCSD}+\mathrm{TQ} *(\mathrm{CCSD})$ & $\mathrm{x}$ & & & \\
\hline $\mathrm{CCSDT}+\mathrm{Q}(\mathrm{CCSDT})$ & $\mathrm{x}$ & & & \\
\hline CCSDT(Q) & $\mathrm{xx}$ & & & \\
\hline $\operatorname{CCSDT}(\mathrm{Q}) / \mathrm{A}$ & $\mathrm{x}$ & & & differs from $\operatorname{CCSDT}(\mathrm{Q})$ for closed-shell non-HF reference \\
\hline $\operatorname{CCSDT}(\mathrm{Q}) / \mathrm{B}$ & $\mathrm{x}$ & & & differs from $\operatorname{CCSDT}(\mathrm{Q})$ for closed-shell non-HF reference \\
\hline$\Lambda$-CCSDT $(\mathrm{Q})$ & $\mathrm{x}$ & & & \\
\hline $\operatorname{CCSD}(\mathrm{T}-n), n=2,3,4,5^{\mathrm{b}}$ & $\mathrm{x}$ & & & \\
\hline $\operatorname{CCSD}(\mathrm{TQ}-n), n=2,3,4^{\mathrm{c}}$ & $\mathrm{x}$ & & & \\
\hline $\operatorname{CCSDT}(\mathrm{Q}-n), n=2,3,4,5,6^{\mathrm{c}}$ & $\mathrm{x}$ & & & \\
\hline $\mathrm{LCCD}^{\mathrm{d}}$ & $\mathrm{x}$ & $\mathrm{x}$ & & \\
\hline $\operatorname{LCCSD}^{\mathrm{e}}$ & $\mathrm{x}$ & $\mathrm{x}$ & & \\
\hline CISD & $\mathrm{x}$ & $\mathrm{x}$ & $\mathrm{x}$ & \\
\hline QCISD & $\mathrm{xxx}$ & $\mathrm{xxx}$ & & \\
\hline QCISD(T) & $\mathrm{xxx}$ & $\mathrm{xxx}$ & & \\
\hline
\end{tabular}

${ }^{a}$ Additional methods, in particular open-shell variants of higher-order coupled cluster methods including in many cases gradients and analytic second derivatives are available through the interface to the MRCC program. See the MRCC manual (www.mrcc.hu) for a complete list.

b See Ref. 107.

c See Ref. 135.

d LCCD stands for linearized CCD.

${ }^{\mathrm{e}}$ LCCSD stands for linearized CCSD. 
analytic closed-shell CCSD gradients. A modern formulation of CC derivative theory is based on the Lagrangian formalism introduced by Helgaker and Jørgensen. ${ }^{148-150}$ In order to cope here with the non-variational character of the standard CC theory, a Lagrangian $L$ is introduced which consists of the CC energy augmented by the CC equations (as the so-called constraints) premultiplied with Lagrange multipliers

$$
L=\langle 0|(1+\Lambda) \exp (-T) H \exp (T)| 0\rangle .
$$

In this equation, a compact notation is used in which the Lagrange multipliers are subsumed into the $\Lambda$ operator, a de-excitation operator that gathers all of them. At this point it should be mentioned that this CC energy functional was actually first suggested by Arponen ${ }^{151}$ in order to cast $\mathrm{CC}$ theory in a variational framework. The Lagrangian is then made stationary. Stationarity with respect to the amplitudes in the $\Lambda$ operator recovers the $\mathrm{CC}$ amplitude equations, while stationarity with respect to the amplitudes in the cluster operator leads to the linear equations for the amplitudes of the $\Lambda$ operator

$$
\left\langle 0|(1+\Lambda)(\exp (-T) H \exp (T)-E)| \Phi_{P}\right\rangle=0 .
$$

Due to the stationarity of $L$, differentiation with respect to a perturbation $x$ yields for the derivative

$$
\frac{d E}{d x}=\frac{\partial L}{\partial x}=\left\langle 0\left|(1+\Lambda) \exp (-T) \frac{d H}{d x} \exp (T)\right| 0\right\rangle
$$

which forms the basis of CC gradient theory.

The discussion so far has ignored orbital relaxation. The consideration of this effect requires coupled-perturbed HF theory, ${ }^{152,153}$ but is in CC gradient theory treated using the $Z$-vector approach by Handy and Schaefer. ${ }^{154}$ CFOUR is able to handle orbital relaxation for RHF and UHF reference functions, ${ }^{5}$ and also in the case of ROHF and some classes of QRHF reference determinants. ${ }^{138,139}$

All analytic gradient implementations in CFOUR (see Table I for methods marked " $x x$ " or " $x x x ")$ make use of a density-based formulation of the first derivatives ${ }^{155,156}$ such that in the final step the perturbation-independent quantities, i.e., the one- and two-particle density matrices as well as some intermediates, are contracted with the derivatives of the one- and two-electron AO integrals without any need to store the latter.

Analytic second derivatives have been formulated and implemented within CC theory. ${ }^{23,30-32,46,157,158}$ CFOUR offers here a range of options with all implementations based on the so-called asymmetric formulation ${ }^{33,159}$ that results from a straightforward differentiation of the gradient expression 
given in Eq. (8) with respect to a second perturbation $y$. This means that the first derivatives of $T$ and $\Lambda$ need to be computed but at no point are these quantities required for two different perturbations at the same time.

Geometrical analytic second derivatives allow the computation of quadratic force constants (and thus harmonic vibrational frequencies) and, via numerical differentiation, ${ }^{34,160}$ of cubic and semidiagonal quartic force constants (and thus in the framework of second order vibrational perturbation theory (VPT2) anharmonic corrections to vibrational frequencies, i.e, the computation of fundamental frequencies as well as the frequencies of overtone and combination bands). ${ }^{67}$ CFOUR offers the corresponding capabilities and renders such computations doable on a routine basis. Corresponding computations can furthermore be easily performed in a parallel manner. We also note that CFOUR offers capabilities to perform such calculations on the basis of numerical differentiation of analytically evaluated forces as well.

Table I also summarizes the CC and MBPT/MP methods for which analytic second derivatives are available in CFOUR (methods marked with "xxx"). Note that so far no analytic second derivatives are available for schemes based on a ROHF reference function.

Analytic differentiation schemes are particularly useful for the computation of the corresponding geometrical derivatives. However, analytic derivatives also provide access to a range of other properties. To be mentioned here in the context of first derivatives are the accessible first-order properties such as dipole moment, quadrupole moment, nuclear electric-field gradients, etc.

There is an additional point to be discussed here, namely whether these first-order properties are computed with or without orbital relaxation effects included. CFOUR offers both options and it has been argued ${ }^{161}$ that CC theory takes (via single excitations) care of orbital relaxation effects $^{162}$ in an adequate manner.

The issue of orbital relaxation is also of relevance when dealing with frequency-dependent properties in the framework of CC response theory. ${ }^{163}$ The consideration of orbital relaxation can lead here to artificial poles and is therefore avoided. CFOUR offers, based on existing analytic second derivative technology, access to frequency-dependent polarizabilities at CCSD level, ${ }^{164}$ $\mathrm{CC} 3,{ }^{163}$ and CCSDT level. ${ }^{165}$ In addition, using third derivatives, frequency-dependent hyperpolarizabilities can be evaluated at the same levels of theory. ${ }^{166-168}$ Further analytic third derivatives include Raman intensities computed as gradients of the frequency-dependent polarizability at the CCSD level ${ }^{169}$ and Verdet constants computed as quadratic response function at the CCSD and CCSDT levels of theory. ${ }^{170}$ 
Concerning the computation of magnetic properties, i.e., nuclear magnetic shielding tensors and magnetizabilities, care has to be taken with respect to the gauge-origin problem. As amply demonstrated in the literature, the use of gauge-including atomic orbitals (GIAOs, ${ }^{171-174}$ also known as London orbitals ${ }^{175}$ ) is here an adequate choice and they are hence used by default in CFOUR. CFOUR offers unique capabilities to compute magnetic properties at various $\mathrm{CC}$ levels with high accuracy for both nuclear magnetic shielding constants ${ }^{20-23,46}$ as well as magnetizabilities. ${ }^{176}$ The implementation of shielding constants at the MP2 level ${ }^{17,18}$ in CFOUR was the first presented in the literature, but by now this option is also offered by other quantum chemical program packages ${ }^{177-180}$ together with advancements that facilitate large-scale calculations. The capabilities of CFOUR concerning the computation of magnetic properties also allow the computation of closely related properties such as nuclear spin-rotation and rotational $g$ tensors ${ }^{181}$ via the use of so-called rotational London orbitals. ${ }^{38}$ In the context of NMR properties, we also note that the second derivative capabilities of CFOUR allow the computation of indirect spin-spin coupling constants at CCSD $,{ }^{182} \mathrm{CC} 3,{ }^{183}$ and CCSDT and higher CC levels (both via MRCC). To be noted here is that (a) these calculations must be performed in an orbital-unrelaxed manner ${ }^{182}$ and that (b) CFOUR allows the computation of all four contributions to the indirect spin-spin coupling constants (i.e., Fermi-Contact, spin-dipole, paramagnetic spin-orbit, and diamagnetic spin-orbit terms). ${ }^{184,185}$

To conclude this section, we mention that CFOUR also offers the capability to compute vibrational corrections to a range of properties via VPT2. ${ }^{186}$ These corrections turn out to be essential in the case of high-accuracy computations that are compared to experimental values from precise gas-phase measurements.

\section{Excited state treatments via equation-of-motion/linear response methods}

Single-reference methods based on MBPT and CC theory are excellent approaches to study the potential energy surfaces associated with ground electronic states near their equilibrium structure, but generally cannot be straightforwardly applied to study excited states. In particular, all such methods are subject to variational collapse (through the reference function $|0\rangle$ ) or convergence to lower-lying states with the same (spatial and spin) symmetry. For closed-shell systems, the lowest singlet excited states often have a symmetry different than the ground state (for example, the lowest excited state of formaldehyde has ${ }^{1} A_{2}$ symmetry while the ground state has ${ }^{1} A_{1}$ symmetry), 
but such states are described (in zeroth order) by a linear combination of two Slater determinants and therefore not amenable to standard MBPT or CC calculations. For many radicals, however, excited states are properly described by a single determinant (for example, the excited ${ }^{2} \Sigma$ state of $\mathrm{OH}$ ), and the usual toolkit of "ground state" MBPT/CC methods can indeed be employed. The same holds for excited triplet states where a single determinant is often a valid description for the high-spin components. However, when one speaks generally of excited states in the context of quantum chemistry, it can be assumed that standard single-determinant methods are not suitable.

The major advance in extending CC theory to excited states was identified in an insightful paper by Monkhorst, ${ }^{187}$ and has ultimately come to be known as both "equation-of-motion CC" (EOM-CC) theory ${ }^{24,75,188,189}$ and "linear response CC" (LR-CC) $)^{190-194}$ theory. Both of these approaches give the same excitation and final state energies (see below), but differ in the way that certain properties are defined (see next subsection). It should be noted that the symmetryadapted-cluster configuration-interaction (SAC-CI) method, ${ }^{195-198}$ which is similar in spirit to the EOM-CC approach, was developed for excited, ionized, and electron-attached states by Nakatsuji and Hirao in the late 70's.

In EOM-CC methods, the final state energies are obtained by diagonalization of the similaritytransformed Hamiltonian $\bar{H}$

$$
\bar{H} \equiv \exp (-T) H \exp (T)
$$

a non-Hermitian operator that is obtained from the usual electronic Hamiltonian using the CC amplitudes in the transformation step. The excited states are described by the wavefunctions

$$
\begin{aligned}
& \left|\Psi_{E O M-C C}\right\rangle=\mathscr{R} \exp (T)|0\rangle \\
& \left\langle\tilde{\Psi}_{E O M-C C}\right|=\langle 0| \mathscr{L} \exp (-T),
\end{aligned}
$$

where $\mathscr{R}$ and $\mathscr{L}$ are the right and left eigenvectors of $\bar{H}$.

The characterization of EOM-CC above applies strictly only to "complete" CC methods like CCSD, CCSDT, etc., but must be modified somewhat for methods in which certain classes of excitation are not treated completely (CC2, ${ }^{102}$ CCSDT- $1,{ }^{92,98}$ and so on). In such a case, the excitation energies are obtained again by diagonalization of a non-symmetric matrix, but one that cannot be written as $\bar{H}$ is designated above. Rather, one differentiates the CC amplitude equations (Eq. (4) of the previous section), which leads to the linear equation

$$
\frac{d T}{d x}=A^{-1} b^{x},
$$


in which $A$ is the "CC Jacobian" that is diagonalized to obtain the excitation energies. This perspective on EOM-CC applies equally well to the normal (CCSD, CCSDT, etc.) case, in which $A=\bar{H}$, and is illuminating in that one can easily see the correspondence between the eigenvalues of $A$ and the excitation energies from the point of view of first-order perturbation theory.

The first EOM-CC calculations were based on the CCSD approximation, and appeared more than thirty years ago, ${ }^{191}$ but the method began to gain momentum with a flurry of activity that took place both in Gainesville and Aarhus after 1990. ${ }^{24,189,193}$ For excited states that can be characterized as "single excitations", EOM-CCSD theory gives excitation energies that are usually no more than $0.25 \mathrm{eV}$ in error, and tends towards overestimation. ${ }^{199-201}$ Later developments led to EOMCCSDT $^{202-204}$ and EOM-CCSDTQ, ${ }^{205,206}$ as well as general arbitrary-order EOM-CC ${ }^{47}$ via the MRCC package. ${ }^{42}$ With these methods, excitation energies become systematically more accurate as the cost of calculation grows significantly. As for ground-state methods, the high cost of EOM-CCSDT calculations has driven efforts to find suitable approximations, and this remains an area of important research. Such approximations include generalizations of the CCSDT- $n$ methods mentioned earlier, CC3 - which is probably the most popular and perhaps successful such approach, ${ }^{207}$ and a great variety of non-iterative methods. While many such methods have been identified and tested, ${ }^{28,202,208-218}$ a recent non-iterative technique (EOM-CCSD(T)(a)* ${ }^{29}$ shows considerable promise, ${ }^{200,219-221}$ and might be the method of choice for future applications.

While sometimes thought of as strictly a means to compute excitation energies, EOM-CC methods also can be used to compute states that differ from the ground state in terms of the number of electrons. That is, their domain of application includes "excited states" in which electrons are "excited" to the continuum (ionization) or electrons are excited from the continuum (electron attachment). EOM-CC methods belonging to the former class are called EOMIP-CC ${ }^{27}$ (removal of one electron), EOMDIP-CC 222 (two-electrons), etc., while those in the latter class are EOMEA$\mathrm{CC}^{223}$ (attachment of one electron), EOMDEA-CC, and so on. EOM-CC methods in which the number of electrons in the initial and final state are identical are then called EOMEE-CC (EE standing for excitation energy). CFOUR has extensive capabilities for EOM-CC calculations for all the variants mentioned above (EOMEE-CC, EOM(D)IP-CC, and EOMEA-CC), the state of which is summarized in Table II.

It should be noted that the capabilities indicated in the table are only for efficient implementations of the methods. This is important because it has been shown ${ }^{224}$ that an EOMEE-CC code can be used to do EOM(D)IP-CC or EOMEA-CC calculations by making use of continuum orbitals; 
TABLE II. EOM-CC methods available in the CFOUR program package for closed-shell reference functions. A single $\mathrm{x}$ designates that only energy evaluations are possible, while $\mathrm{xx}$ indicates that both energy and gradients can be calculated. The MRCC extension also does general CC(n) (n>1) energies and gradients for open- and closed-shell references.

\begin{tabular}{|c|c|c|c|c|}
\hline method & EOMEE & EOMIP & EOMEA & remarks \\
\hline CCSD & $\mathrm{xx}$ & $\mathrm{xx}$ & $\mathrm{xx}$ & also open-shell $|0\rangle$ and EOMDIP \\
\hline CCSDT & $\mathrm{xx}$ & $\mathrm{x}$ & & also EOMDIP \\
\hline CCSDTQ & $\mathrm{x}$ & $\mathrm{x}$ & & \\
\hline CCSDT $-n, n=1 \mathrm{a}, 1 \mathrm{~b}, 2,3$ & $\mathrm{x}$ & & & \\
\hline $\mathrm{CC} 2$ & $\mathrm{xx}$ & $\mathrm{x}$ & & inefficient code, $M^{6}$ scaling \\
\hline $\mathrm{CC} 3$ & $\mathrm{x}$ & & & \\
\hline $\mathrm{CCSD}^{* \mathrm{a}}$ & $\mathrm{x}$ & $\mathrm{x}$ & & \\
\hline $\operatorname{CCSD}(\mathrm{T})^{b}$ & $\mathrm{x}$ & & & \\
\hline $\operatorname{CCSD}(\mathrm{T})(\mathrm{a})^{\mathrm{c}}$ & $\mathrm{x}$ & $\mathrm{x}$ & & \\
\hline $\operatorname{CCSD}(\mathrm{T})(\mathrm{a}) * \mathrm{c}$ & $\mathrm{x}$ & & & \\
\hline $\operatorname{CCSDR}(n), n=\mathrm{T}, 1 \mathrm{a}, 1 \mathrm{~b}, 3^{\mathrm{d}}$ & $\mathrm{x}$ & & & \\
\hline CIS & $\mathrm{xx}$ & & & \\
\hline CIS(D) & $\mathrm{xx}$ & & & \\
\hline $\operatorname{CCSD}(2)$ & $\mathrm{xx}$ & $\mathrm{xx}$ & & \\
\hline
\end{tabular}

\footnotetext{
a See Ref. 28.

${ }^{\mathrm{b}}$ See Ref. 214.

c See Ref. 29.

${ }^{d}$ See Ref. 210.
}

excitation of one electron to this continuum orbital is equivalent to EOMIP-CC, excitation from an occupied continuum orbital is equivalent to EOMEA-CC, etc. That is, while the table indicates that, for example, EOMEA-CCSDT is not "available" in CFOUR, such calculations can indeed be done by this means, although the resulting implementation has the same cost as the corresponding EOMEE-CCSDT calculation. CFOUR allows the straightforward use of these continuum orbital 
techniques, and the capabilities extend to both energy and gradient calculations.

In addition to EOMEE-CC methods, CFOUR is also able to perform calculations using configuration interaction singles ${ }^{225}$ (CIS, also known as the Tamm-Damcoff approximation ${ }^{226,227}$ ), the perturbatively corrected CIS(D) method, ${ }^{228}$ and an approximate method known as EOM$\operatorname{CCSD}(2) .{ }^{229}$ All of these methods work at the excitation energy level, and both EOMEE-CCSD(2) and EOMIP-CCSD(2) are implemented.

Several functionalities are available to direct the program into the desired excited state. The character of the excitation can be specified in terms of dominant orbitals as further explained in Section III. Alternatively, one can simply request the lowest excited state(s) of a particular spin and spatial symmetry. It is also possible with CFOUR to compute excited states near a particular target energy.

\section{Analytic derivatives and properties for excited states}

While the pioneering work with EOM-CC theory dealt strictly with energy differences (vertical excitation energies, ionization potentials and electron attachment energies), the central importance of excited states in chemical physics has demanded that the associated potential energy surfaces be characterized computationally. Such studies are relevant not only for analysis and predictions of electronic spectroscopy, but also to study photochemical behavior and interactions between excited states. Accordingly, analytic derivative techniques similar in the spirit of application to those mentioned in section IIB were developed for EOM-CC methods in the early $1990 \mathrm{~s}^{25-27}$ and were present in the first version of CFOUR. The EOM-CC energy gradient is given by

$$
\frac{d E}{d x}=\left\langle 0\left|\mathscr{L} \frac{\partial \bar{H}}{\partial x} \mathscr{R}\right| 0\right\rangle+\left\langle 0\left|\mathscr{Z} \frac{\partial \bar{H}}{\partial x}\right| 0\right\rangle,
$$

and, apart from contractions between the differentiated electronic Hamiltonian and the right- and left-eigenvectors of $\bar{H}$ (note that a calculation of the excitation energies requires only that one of these eigenvectors be evaluated), involves an additional de-excitation operator $\mathscr{Z}$, which is analogous to the $\Lambda$ operator in ground-state gradient theory. The amplitudes that make up this operator are obtained from solving the linear system

$$
\left\langle 0|\mathscr{Z}| \Phi_{P}\right\rangle=\left\langle 0|\Xi| \Phi_{P}\right\rangle\left[\left\langle 0\left|\bar{H}-E_{C C}\right| 0\right\rangle\right]^{-1},
$$

where matrix elements of the auxiliary operator $\Xi$ are defined by

$$
\left\langle 0|\Xi| \Phi_{P}\right\rangle \equiv\left\langle 0|\mathscr{L} \bar{H}| \Phi_{Q}\right\rangle\left\langle\Phi_{Q}|\mathscr{R}| \Phi_{P}\right\rangle
$$


with $\Phi_{Q}$ representing a determinant in the space of excitations beyond that defined by the particular truncated CC approach (for example, triple excited determinants in CCSD).

As for ground state CC methods, the general gradient formula (Eq. 13) is recast in terms of one- and two-electron density matrices. Contraction of these with the geometric derivatives of the Hamiltonian gives the gradient, while contraction of the densities with other operators again provides other properties. EOM-CCSD and EOM-CC2 gradients are available in CFOUR for all methods (EOMEE, EOMIP and EOMEA), for both closed-shell and open-shell reference functions, and offer a very efficient means to study potential energy surfaces of the final states. EOMEE-CCSDT gradients for closed-shell references are a very recent addition, and general EOMEE-CC $(n)$ gradients are available with the MRCC interface. It is a straightforward matter here to calculate properties such as dipole moments, higher multipole moments, Mulliken populations, and so on, using the one-electron density; these properties are all equivalent to those calculated as energy derivatives.

In addition to gradients, one-electron transition densities involving only the ground-state $T$ amplitudes and the $\mathscr{L}$ and $\mathscr{R}$ vectors ${ }^{25}$ are available. These yield, among other things, transition moments. And it is here (and only here) that EOM-CC and CCLR methods provide different results. ${ }^{230-232}$ The transition moments evaluated in CFOUR calculations - those mentioned here - are not size-intensive, becoming so only in the limit of a full CC (i.e. CCSDTQ for a fourelectron system) calculation. In CCLR theory, the transition moments satisfy size-intensivity, but involve the cost associated with solving an additional set of linear equations for each excited state considered.

\section{INPUT AND USE OF CFOUR}

CFOUR calculations are rather straightforward to perform. After having installed CFOUR (for information concerning the installation of CFOUR, see the CFOUR website www.cfour.de and Appendix 1) and with all executables placed either in the working directory of the calculation or in a directory (e.g., . / cfour/bin/) that is part of the path, all calculations (unless otherwise advised) are invoked by the command xcfour. This command calls a driver program which, after having analyzed the input file ZMAT (see below), determines the various modules that need to be run, and in what order.

The input for a CFOUR calculation consists of a single file. This file, called ZMAT, consists of 
several sections as shown in the following example. The first three sections are always necessary, while the fourth is optional (dependent on the chosen computational approach).

EOMEE-CCSD/cc-pVDZ calculation for water

$\mathrm{H}$

$01 \mathrm{R}$

$\begin{array}{lllllllll}H & 1 & R & & A\end{array}$

$\mathrm{R}=0.958$

$A=104.5$

*CFOUR $($ CALC=CCSD , BASIS=PVDZ , EXCITE=EOMEE $)$

\%excite*

1

1

15506001.0

The ZMAT file starts with a mandatory one-line title, which is followed by the geometry information, either in Z-matrix format (shown here, and which is currently mandatory for geometry optimizations) or in Cartesian coordinates. The geometry is followed by a list of keywords in a sequence of lines that starts with $*$ CFOUR. There are roughly 250 active keywords, but virtually all of them take on default values (or are modified by default according to other keywords in the input file). Common keywords to supply, as shown in the example file above, include information about the chosen quantum chemical method (CALC=CCSD obviously invokes a CCSD calculations), basis set (BASIS=PVDZ requests the use of the cc-pVDZ basis) and calculation type (EXCITATION=EOMEE requests an EOMEE-CCSD treatment). Additional parameters such as convergence thresholds, maximum number of iterations, etc. can also be modified, but they have appropriate default values and do not need to be supplied. The final section (initiated by a \% sign) provides additional information. In the example chosen here, this information guides the choice of guess vectors for the EOMEE-CCSD computation, with this particular example instructing the EOM-CC program to start with the HOMO $\rightarrow$ LUMO guess in the Davidson diagonalization procedure. 
Basis set information is provided via the file GENBAS which can either be customized and externally supplied or used from the default location (. ./cfour/basis/). The same holds also for information about effective core potentials (ECP), which is supplied via the related file ECPDATA.

Of course, more elaborate input files can be created, and it is sometimes advantageous or necessary to include additional files (beyond ZMAT) in the running directory. Examples here include the file FCMINT (which contains the force constants in Z-matrix internal coordinates), which can be supplied to facilitate geometry optimization (this permits the force constants in FCMINT to be used as a starting guess for the Hessian as opposed to a naive set of initial parameters). The ZMAT file below,

Calculation of LVC parameters for nitrogendioxide

0

N 1 R

$02 \mathrm{R} 1 \mathrm{~A}$

$\mathrm{R}=1.26239$

$\mathrm{A}=116.4431$

$*$ CFOUR $($ CALC $=$ CCSD , BASIS $=$ AUG $-P V D Z$, FROZEN_CORE=ON

EXCITE $=$ EOMIP, SCF_EXPSTART $=10$

CC_MAXCYC $=200$, LINEQ_MAXCYC $=200$

FCGRADNEW $=0$

CHARGE $=-1$

TRANGRAD $=0 \mathrm{~N}$, DERIV_LEV $=1$ )

\%excite*

1

1

10010001.0

together with the file FCMFINAL, which, in this example, contains the force constants for the $\mathrm{NO}_{2}$ anion, calculated separately), provides the input to calculate the linear LVC parameters in Table IX (vide infra) for the $\tilde{A}^{2} B_{2}$ state (the $\kappa_{s}^{A}$, vide infra). In addition to directing CFOUR to do 
an EOMIP calculation with the $\mathrm{NO}_{2}$ anion as reference, it specifies the calculation of a gradient (DERIV_LEV=1), that this gradient should be transformed to the normal coordinate representation associated with the force constants in FCMFINAL, that the frozen core approximation is to be used, and also some other parameters about the algorithm used for the frozen-core gradient calculation, and specifications for the maximum number of cycles for various equations that are solved.

Clearly, it is not possible or appropriate here to give an exhaustive list of examples. The point is simply to show a few representative cases, and to state that the input is generally quite simple: the ZMAT file and perhaps another file or two, depending on the type of calculation. More examples can be found on the CFOUR website (see Appendix 1).

\section{NEW FEATURES}

\section{A. Higher-Order Coupled Cluster Methods: xncc}

Highly-accurate calculations often require treatment of the correlation energy beyond $\operatorname{CCSD}(\mathrm{T})$. For example, many common thermochemical protocols such as HEAT, ${ }^{233-235} \mathrm{Wn},{ }^{236-238}$ and $\mathrm{ANL}{ }^{239}$ include not only CCSDT contributions but additional contributions from quadruple excitations (CCSDT(Q) or CCSDTQ) and in some cases even quintuple excitations (CCSDTQ(P) or CCSDTQP). Such corrections are critical (in combination with corrections for relativistic effects, basis set convergence, etc. described in other sections) to reaching sub-kJ/mol accuracy, and enabling real-world applications using these methods has long been a design goal of CFOUR.

For many years, CFOUR has supported CCSDT energy calculations for both closed and openshell references, as well as properties, gradients, and even second derivatives at the closed-shell CCSDT level. Additionally, the CCSDT(Q) method, ${ }^{108}$ which provides a cost-effective and often highly-accurate approximation to full CCSDTQ was originally implemented in a development version of CFOUR. More recently, the full hierarchy of coupled cluster methods has been made accessible via the interface between CFOUR and the MRCC program of Kállay. ${ }^{44}$

However, in the last several years we have become interested in writing a new implementation of CCSDT(Q), CCSDTQ, and other higher-order coupled cluster methods that maximizes efficiency and scalability on modern computers, as well as developing new theoretical techniques to facilitate such an implementation. For closed-shell references, we developed a general algebraic and graphical interpretation of the non-orthogonal spin-adaptation approach ${ }^{240,241}$ first pioneered 
TABLE III. Timing of CCSDT(Q) and CCSDTQ calculations in minutes (from Ref. 62) for a representative set of small molecules. Two basis sets are listed for some molecules, in this case the first basis set refers to the $\operatorname{CCSDT}(\mathrm{Q})$ calculation while the second refers to the CCSDTQ calculation. The time for the CCSDT part (per iteration) and the (Q) correction in $\operatorname{CCSDT}(\mathrm{Q})$ are listed separately.

\begin{tabular}{ccccc}
\hline \hline & & CCSDT & (Q) & CCSDTQ \\
\hline $\mathrm{HSOH}$ & cc-pVTZ/cc-pVDZ & 3.7 & 85.5 & 9.3 \\
$\mathrm{H}_{2} \mathrm{O}$ & cc-pVQZ/aug-cc-pVTZ & 0.3 & 5.9 & 19.7 \\
$\mathrm{H}_{2} \mathrm{CCCCH}_{2}$ & cc-pVDZ/DZ & 1.2 & 43.9 & 35.1 \\
$\mathrm{O}_{3}$ & aug-cc-pVDZ & 0.2 & 7.5 & 99.6 \\
$\mathrm{FO}_{3}{ }^{-}$ & cc-pVDZ & 0.5 & 12.3 & 241.3 \\
\hline \hline
\end{tabular}

by Kucharski and Bartlett, ${ }^{242}$ and later used by one of us (JG) to develop an efficient closedshell CCSDT code in CFOUR. In order to maximize efficiency, we coupled this mathematical technique with a storage format and set of implementation techniques designed to minimize data movement (from disk as well as from main memory) and to avoid costly tensor transposes. ${ }^{62} \mathrm{We}$ also made code quality a major design goal, and we put a large focus on modularity and code reusability, maintainability, and extensibility. Finally, we included explicit OpenMP parallelization to effectively make use of modern multi-core processors.

The end product of this work is a new CFOUR module, xncc, ${ }^{62,240}$ which implements a full suite of coupled cluster methods for closed-shell molecules through CCSDTQ, including in most cases gradients (see Table I for the full list of supported methods). Calculations with xncc can be requested with CC_PROGRAM=NCC, but in most cases this is not necessary as xncc is the default program for CCSDT(Q) and CCSDTQ. Sample timings from Ref. 62 are listed in Table III as the number of minutes per iteration (for CCSDT and CCSDTQ) or the time in minutes required for the $(\mathrm{Q})$ correction. The hardware used here was one core of an Intel Xeon E5620 processor with $22 \mathrm{GiB}$ of memory allocated to CFOUR. From these results it is immediately clear that significant speed-ups can be achieved with xncc compared to other programs-while these results use only one core, the multi-core scalability of xncc is also very good, with parallel efficiencies (achieved parallel speed-up divided by number of cores used) of $\sim 50 \%$ for 8 or more cores.

xncc also includes implementations of EOMEE-CC and EOMIP-CC methods through CCSDTQ, with gradients available for EOMEE-CCSD and EOMEE-CCSDT. In lieu of full EOMEE- 
CCSDT, a number of approximate methods are also included: EOMEE-CCSD* ${ }^{28,243}$-CCSD(T)(a) and $-\operatorname{CCSD}(\mathrm{T})(\mathrm{a}) *{ }^{29}-\mathrm{CC} 3,{ }^{207}-\mathrm{CCSDT}-n$ and $-\operatorname{CCSD}(\mathrm{T}),{ }^{209,211}$ and $-\operatorname{CCSDR}(\mathrm{T}),-\operatorname{CCSDR}(1 \mathrm{a})$, and -CCSDR(3). ${ }^{210}$ Corrections to excited state energies, geometries, and vibrational frequencies can be rather large; for example in a calculation of the geometries and harmonic frequencies of the $S_{1}$ excited state potential energy surface of $\mathrm{C}_{2} \mathrm{H}_{2}$, we found that triples contributions to the harmonic frequencies can be in excess of $100 \mathrm{~cm}^{-1}$ while quadruples corrections can be as large as $35 \mathrm{~cm}^{-1}{ }^{206}$ While the current release includes analytic gradients for EOMEE-CCSDT, transition properties at this level have not yet been implemented, but will be included in the next version along with EOMEE-CCSDT natural transition orbitals.

Another unique feature of xncc is the use of sub-iteration convergence acceleration for the CCSDT, CCSDTQ, and approximate CCSDT (CC3 and CCSDT- $n$ ) methods. ${ }^{244}$ For CCSDT and other iterative triples methods, this technique essentially "freezes" the higher-order cluster amplitudes and their contributions to the singles and doubles while a number of (modified) CCSD iterations are performed. The triples amplitudes are then updated and the cycle repeats. For CCSDTQ, two levels of sub-iteration are possible and xncc utilizes both of them simultaneously by default. For all methods, but especially for approximate methods such as CC $n$, CCSDT- $n$, and CCSDTQ- $n$, this technique can drastically reduce the number of iterations required for convergence. The current version includes sub-iteration for the amplitude equations, optional DIIS for the triples and/or quadruples amplitudes, as well as optional amplitude damping that can help in cases where oscillatory behavior is encountered. The next version will extend the sub-iteration technique to linear equations (e.g. the $\Lambda$ equations) and potentially to EOM-CC as well.

The availability of a high-performance yet easily extensible platform for higher-order coupled cluster has also allowed us to rapidly implement new coupled cluster-based methods. Perhaps the best example of this is the recent development of bivariational coupled cluster perturbation theory methods CCSD(T- $n)$, CCSD(TQ- $n$ ), and CCSDT(Q- $n),{ }^{107,135}$ for which we have implemented up to $n=5,4$, and 6 , respectively. These methods, with the exception of the lowest-order correction, scale formally the same as the full method (CCSDT or CCSDTQ), but, by recovering essentially all of the higher-order correlation energy in only a small number of high-scaling steps, a steep reduction in computational cost can be achieved. As an example, errors in total atomization energies for a test set of small molecules with respect to full CCSDTQ are summarized in Table IV. ${ }^{135}$ From these results, we can see that CCSDT(Q-3) can reduce errors by approximately one order of magnitude compared to $\operatorname{CCSDT}(\mathrm{Q})$ at the expense of one $M^{10}$ step. 
TABLE IV. Total atomization energy errors w.r.t. CCSDTQ in $\mathrm{kJ} / \mathrm{mol}$ for various approximate quadruples methods (from Ref. 135). Errors are summarized by Mean Signed Error, Mean Absolute Error, and MAXimum-amplitude signed error.

\begin{tabular}{cccc}
\hline \hline & CCSDT & CCSDT(Q) & $\Lambda$-CCSDT(Q) \\
\hline MSE & -3.06 & 0.55 & 0.35 \\
MAE & 3.06 & 0.56 & 0.36 \\
MAX & -14.06 & 4.01 & 1.92 \\
\hline \hline & CCSDT(Q-2) & CCSDT(Q-3) & CCSDT(Q-4) \\
\hline MSE & -0.70 & -0.01 & -0.15 \\
MAE & 0.70 & 0.08 & 0.15 \\
MAX & -2.58 & -0.29 & -0.97 \\
\hline \hline
\end{tabular}

All of the capabilities described here (except where noted) are available in the current version. The next release of xncc will focus on implementing open-shell alternatives for all supported methods, in particular CCSDT(Q) and CCSDTQ. Additionally, the version of xncc under development has included further performance improvements due to transpose-free tensor contraction operations from the TBLIS library, ${ }^{245}$ including extension to tensors with explicit point-group symmetry. ${ }^{246}$ We also hope to include scalable distributed-parallel implementations in the next release.

\section{B. Quadratically convergent SCF and complete active space SCF methods}

A rigorous treatment of multireference systems can usually not be achieved by using a singlereference method (see section II C). In order to have not only a method to describe such systems in an unbiased and qualitatively correct way, but to have also a starting point for internally-contracted multireference correlated treatments, an implementation of the Complete Active Space-SelfConsistent Field (CASSCF) method ${ }^{247,248}$ has been recently added to CFOUR. In CASSCF, the orbital space is partitioned into three groups, namely: i) internal orbitals, which are always doubly occupied, ii) active orbitals, with floating occupation, and iii) external orbitals, that are always empty. The molecular wavefunction is written as the linear combination of all the symmetry allowed Slater determinants that can be formed by varying the occupation of the active orbitals for a 
given number of active electrons. Both the orbitals and the CI coefficients are then fully optimized. Such a non-linear optimization problem is typically difficult to converge and ill-conditioned, making the use of advanced numerical strategies mandatory. Many CASSCF algorithms have been developed in the past. The numerical strategies proposed can be grouped in two main classes depending on their convergence properties, namely, first-order methods ${ }^{249-254}$ and second-order methods. ${ }^{255-261}$ The latter strategy is particularly attractive, because second-order methods offer rigorous convergence results and are particularly robust, so that achieving convergence requires little to no case-by-case calibration by the user.

The implementation strategy pursued for the CASSCF module of CFOUR is based on the Norm Extended Optimization (NEO) algorithm of Jensen and co-workers. ${ }^{77,258-260}$ The CI operations are handled in a direct fashion using a string-based determinant $\mathrm{CI}$ formalism ${ }^{262-264}$ and the CI implementation follows the integral-driven, vector implementation by Bendazzoli et al. ${ }^{265}$

A second-order optimization strategy is based upon the definition of a quadratic model $\mathscr{Q}$ of the energy, obtained by expanding it in Taylor series with respect to the variational parameters $\mathbf{x}$ up to the second order around a starting point $\mathbf{x}_{0}$

$$
\mathscr{Q}(\mathbf{x})=E\left(\mathbf{x}_{0}\right)+\mathbf{g}^{\dagger} \mathbf{x}+\frac{1}{2} \mathbf{x}^{\dagger} \mathbf{G x},
$$

where $\mathbf{g}$ and $\mathbf{G}$ are the energy gradient and Hessian evaluated at the expansion point. The straightforward minimization of the quadratic model corresponds to the Newton-Raphson (NR) method $^{142}$, and prescribes to take a step

$$
\delta^{\mathrm{NR}}=-\mathbf{G}^{-1} \mathbf{g} .
$$

The NR method enjoys quadratic convergence if the starting point is close to a local minimum, but is known to exhibit erratic behavior or even to diverge if, at the starting point, the Hessian is not positive definite. This issue can be solved by defining a trust region, i.e., a maximum stepsize $R_{t}$ within which the quadratic model of the energy is deemed to provide an accurate representation. This constraint can be imposed by means of a Lagrange multiplier $v$. By doing so, one gets, for the step, the following coupled equations

$$
\left\{\begin{array}{l}
(\mathbf{G}+v \mathbf{I}) \boldsymbol{\delta}=-\mathbf{g} \\
\|\boldsymbol{\delta}(v)\|=R_{t}
\end{array}\right.
$$

The trust-radius Netwon method is also known as Levenberg-Marquardt (LM) method. ${ }^{142}$ If the LM method is coupled with an adaptive choice of the trust radius $R_{t}$, as proposed by Fletcher, ${ }^{142}$ 
depending on the agreement of the quadratic model with the energy, it is possible to prove that, under certain regularity hypotheses of the energy that can be assumed to be satisfied, the procedure always converges to the closest local minimum. The NEO algorithm is an elegant practical implementation of the Fletcher-Levenberg-Marquardt (FLM) strategy, thus enjoying its convergence properties. ${ }^{259}$ The NEO scheme is the default for state-specific CASSCF calculations. The implementation in CFOUR also includes another second-order algorithm, in particular, a simplified version of the one proposed by Meyer, Werner, and Knowles, ${ }^{74,256,261}$ which can be used for state-averaged CASSCF. CASSCF calculations are requested via the CALC=CASSCF keyword and require one to provide as an additional input the definition of the orbital spaces. This is done by adding a section to the ZMAT input file that specifies the number of active alpha and beta electrons and the number of active orbitals and then the actual definition of the active space. The latter can be provided in two different ways. The first possibility, invoked with the keyword CAS_INPUT=ORBITALS, is to specify a list of active orbitals (in HF energy order), the second, invoked with the keyword CAS_INPUT=OCCUPATION, by specifying for each irreducible representation the number of internal orbitals and then the number of active orbitals. The following example provides the input for a CASSCF calculation on benzene, in $D_{2 h}$ symmetry, correlating the $6 \pi$ electrons in the $6 \pi$ orbitals, using the first strategy, where the order of the orbitals is obtained from a HF calculation using the cc-pVDZ ${ }^{266}$ basis set

$\% \operatorname{casscf}$

336

$1720212223 \quad 30$

The same calculation, using the second input method, is obtained with the following route

$\% \operatorname{casscf}$

336

6455300000

00000212121

Other options that control the CASSCF calculations can be found in the CFOUR online manual (see Appendix 1). CASSCF can be used with either non-relativistic or spin-free relativistic Hamiltonians, that are detailed in section IV C. At the moment, the CASSCF code is still experimental, and it is thus not included in the current public release of CFOUR. The code will be made available with the next release. 
The quadratically convergent machinery developed for CASSCF can also be employed to deal with a particularly important subcase, i.e., regular SCF. These equations can be notoriously difficult to converge using a standard SCF algorithm even when Pulay's direct inversion in the iterative subspace $^{267}$ (DIIS) is used to accelerate convergence, especially for open-shell systems. Furthermore, even for well-behaved systems, it can be difficult to achieve very tight convergence, which is required for instance when computing numerical derivatives of post-HF Hessians in anharmonic force field calculations. In all these cases, the user must try and adjust a combination of SCF convergence parameters, such as whether to damp the first iterations and what damping parameters to use, how many points to use for the DIIS extrapolation and when to start it. Tuning all these parameters on a system-dependent basis can be very time consuming, especially if one has to perform a large number of calculations, for which different parameters need to be used.

In such a situation, the robust convergence properties of a second-order scheme are particularly useful. A quadratically convergent implementation of restricted and unrestricted HF based on the solution of Eqs. 18 is available in the last public release of CFOUR and can be used by adding the SCF_PROG=QCSCF keyword. The current implementation works in the MO basis and requires to fully assemble and diagonalize the $\mathrm{MO}$ rotation Hessian and is, therefore, much more computationally demanding than regular SCF. However, as HF is typically an intermediate step in a correlated calculation, this is in practice not an issue for the standard CFOUR user. A new, direct, AO-based implementation that uses the NEO algorithm exists and can be accessed by specifying SCF_PROG=DQCSCF. However, such an implementation is not mature enough to be released at the moment, and will be made available with the next release of the code.

The QCSCF program can be considered an almost black-box SCF code. However, there are a few precautions that the user needs to take. The code performs, at the beginning of the calculation, a few regular SCF iterations that are used in order to get a better starting point for the QC solver and, if a calculation is run with symmetry, to try to guess the correct occupation numbers for each irreducible representation. These are fixed during the QC optimization, so that QCSCF will converge to a minimum for that given occupation. The user should therefore make sure that the occupation numbers guessed are correct, or provide the correct ones in input. A second aspect that should be considered is the general conditioning of the problem. If a very large basis set is used, linear dependence problems can be encountered, as it can be seen by looking at the eigenvalues of the overlap matrix. In such cases, it will not be possible to converge the SCF equations beyond a certain threshold due to numerical precision limitations. This issue can be easily detected by 
looking at the QCSCF iterations. If the residual norm starts oscillating or iterations are stagnating, it means that the best numerical solution that can be achieved for the chosen basis set has been reached, and the user should either consider the calculation converged or, if not satisfied with the result, remove redundant basis functions. A third aspect concerns UHF cases for which multiple SCF solutions with different spin contamination exist. QCSCF is guaranteed to converge to the closest local minimum, which might be different from the one found with regular SCF. In the experience of the authors, QCSCF tends to converge to the solution which is lowest in energy and more spin-contaminated. Whether this solution is acceptable is something that the user needs to check. Nevertheless, a subsequent post-HF treatment is usually able to remove most of the spin contamination. An interesting aspect of QCSCF is that, when regular SCF converges to an unstable solution, QCSCF usually manages to converge to a stable one, at least within the symmetry of the electronic wavefunction. However, convergence can be difficult, especially if the MO rotation Hessian has several small and close eigenvalues.

In order to illustrate the robustness of QCSCF, we propose two examples. As an example of a routine application where very tight convergence is required, we compute the SCF solution for benzene ( $\mathrm{C}-\mathrm{C}$ distance $1.3989 \AA, \mathrm{C}-\mathrm{H}$ distance $1.0808 \AA$ ) with the aug-cc-pVTZ ${ }^{266}$ basis set. This is a standard calculation, however, we require the wavefunction to be converged to $10^{-11}$ in the root mean square (RMS) norm of the MO rotation gradient. Using the default parameters for the calculation and starting from a guess obtained by diagonalizing the core Hamiltonian, QCSCF performs 6 regular SCF iterations, until the root mean square (RMS) variation of the density matrix is smaller than 0.1 , and then manages to converge in only 4 FLM iterations. On the other hand, the regular SCF code easily achieves an intermediate convergence (maximum change of the density matrix smaller than $10^{-7}$ ) but then struggles to further refine the solution, exhibiting an oscillating behavior. The convergence profiles of the two algorithms are reported in Figure 1. The superlinear convergence of QCSCF is particularly apparent, as two convergence profiles can be seen focusing on the green line. The regular SCF iterations exhibit a linear convergence profile. As soon as the FLM iterations start, the energy error drops very rapidly until convergence is achieved.

A second, more challenging example concerns a weakly bonded complex of molecular oxygen and argon $\left(\mathrm{O}-\mathrm{O}\right.$ distance $1.25 \AA$, $\mathrm{O}-$ Ar distance $2.1748 \AA, \mathrm{O}-\mathrm{O}-\mathrm{Ar}$ angle $\left.174.21^{\circ}\right)$, the ground state of which is a triplet. For this molecule, described using a UHF reference, the regular SCF code converges with some difficulty to an unstable solution, that has both a UHF-UHF instability that preserves the symmetry of the wavefunction and a UHF-UHF instability to a broken-symmetry 


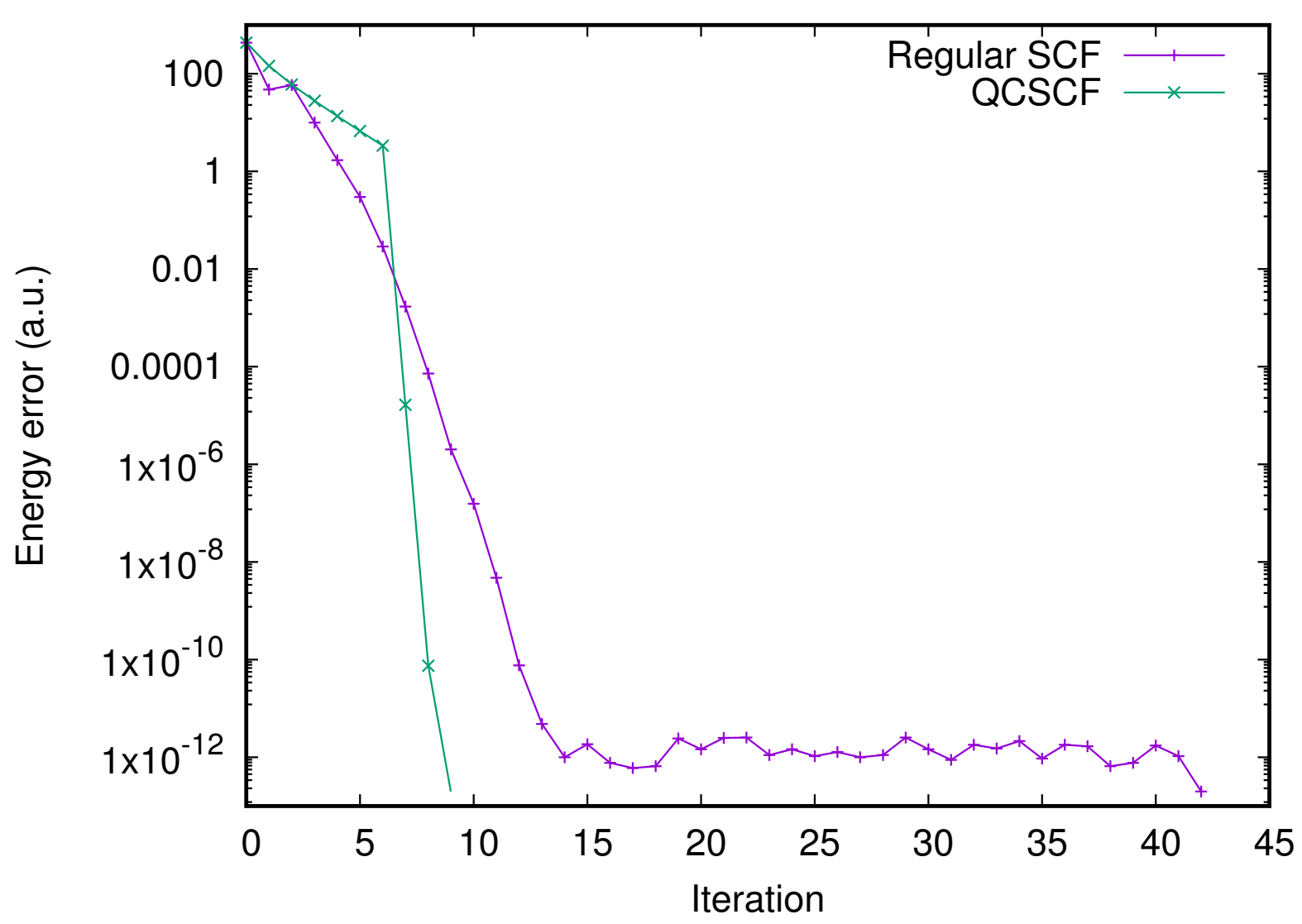

FIG. 1. Convergence profile for the regular SCF code and QCSCF for Benzene. The converged energy is $-230.780571677 E_{h}$.

solution. QCSCF manages to converge to a minimum within the symmetry (about $1 \mu E_{h}$ lower in energy than the regular SCF solution), although convergence requires as many as 36 FLM iterations. An instability with respect to a broken symmetry solution is, however, still present. Interestingly, the NEO based code xdqcscf converges effortlessly to a stable solution—no instability is found even with respect to a broken symmetry UHF solution. While the latter result is a fortunate occurrence that can in general not be expected, the better convergence properties of the NEO based code can be explained by the fact that the NEO algorithm introduces an augmented Hessian, so that the presence of small and close eigenvalues in the original MO rotation hessian has a small effect on the overall convergence of the optimization. The convergence profile of the three algorithms is reported in Figure 2. It is interesting to comment on the behavior of QCSCF. The first iterations manage to quickly locate the same solution found by the regular SCF code. However, the iterations are not stopped as QCSCF detects the instability in the form of a negative eigenvalue 


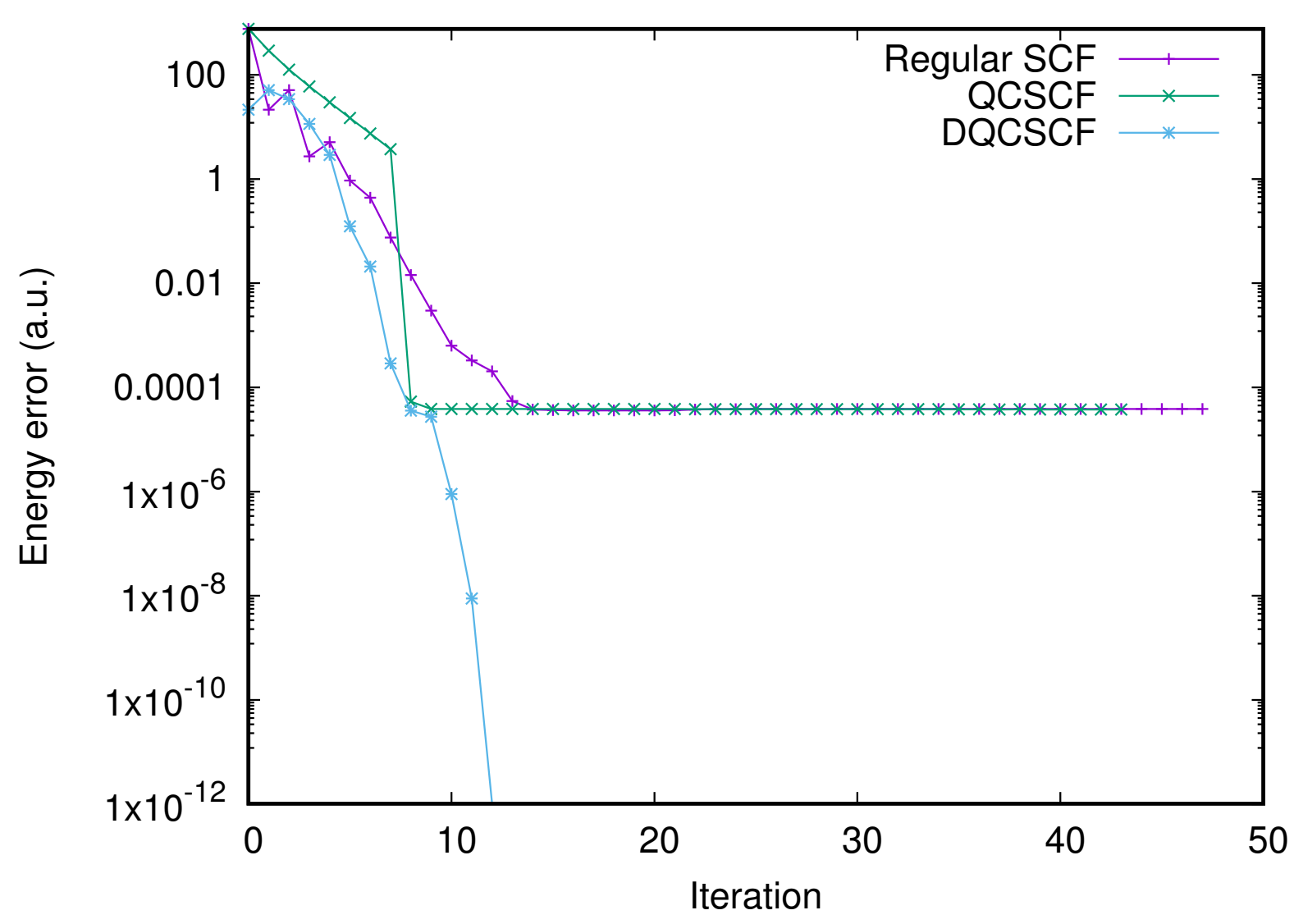

FIG. 2. Convergence profile (absolute energies are reported) for the regular SCF code, the default QCSCF code, xqcscf, and the NEO based code, xdqcscf, for Ar $\cdots \mathrm{O}_{2}$. The converged energy is $-676.39126181 E_{h}$ for the regular SCF, that finds an unstable solution both with respect to a UHF solution with the same symmetry and with broken symmetry, $-676.39126267 E_{h}$ for xqcscf, that finds a solution which is unstable with respect to a UHF solution with broken symmetry and $-676.39130017 E_{h}$ for xdqcscf, that finds a stable solution.

in the MO rotation hessian. A large number of iterations are then spent trying to reach the local minimum. As the lowest eigenvalues of the Hessian are both small and very close, convergence is very slow. On the other hand, the NEO based xdqcscf code does not suffer from this problem and converges smoothly to the global minimum.

\section{Relativistic quantum chemical methods}

Treatment of relativistic effects ${ }^{268,269}$ is indispensable for calculations of molecules containing heavy elements and also plays an important role in high-accuracy calculations of molecules 
that comprise lighter atoms from the first few rows of the periodic table. The development of relativistic quantum-chemical methods in CFOUR has focused on obtaining relativistic corrections to energies and properties with $\mathrm{CC}$ treatment of electron correlation. Initial efforts on perturbative treatment of scalar-relativistic effects were focused on the framework of standard (non-relativistic) CC gradient theory and the Breit-Pauli Hamiltonian. ${ }^{270,271}$ First-order scalarrelativistic corrections to energies can be conveniently obtained in a calculationof first-order properties (PROP=FIRST_ORDER), and are widely used in well-established protocols for computation of thermochemical parameters. ${ }^{233}$ Calculations of scalar-relativistic corrections to geometrical parameters and electrical properties have been enabled by using nonrelativistic analytic CC second-derivative techniques. ${ }^{272}$ Perturbative techniques for treating relativistic effects have been extended to using direct perturbation theory (DPT), ${ }^{52}$ a four-component formalism that permits a rigorous treatment of two-electron contributions. ${ }^{273-275}$ In the released version of CFOUR, the use of a keyword (RELATIVISTIC=DPT2) in geometry optimization and evaluation of first-order properties is a convenient way of obtaining leading relativistic corrections to geometries and firstorder electrical properties. Uncontracted basis sets are recommended for DPT calculations, since DPT requires an accurate description for both the non-relativistic and the relativistic wavefunctions. DPT corrections to energies have been implemented in CFOUR through fourth order with respect to $c^{-1}$ (DPT4) as analytic second derivatives of non-relativistic energies, including both scalar-relativistic corrections and spin-orbit corrections, ${ }^{53}$ and have been further extended to sixth order for scalar-relativistic corrections. ${ }^{276}$ Furthermore, DPT4 corrections to electrical properties can be computed. ${ }^{54}$ The development of DPT has also provided relativistic one- and two-electron integrals required for development of non-perturbative approaches.

Subsequent development of relativistic quantum chemical methods within CFOUR has involved a rigorous non-perturbative treatment of scalar-relativistic effects augmented with a perturbative treatment of spin-orbit coupling. In these calculations, the cost of the coupled-cluster steps of a scalar-relativistic calculation is essentially identical to that of the corresponding nonrelativistic calculation. In contrast, spin-symmetry breaking due to spin-orbit coupling leads to substantial computational overhead; a spin-orbit $\mathrm{CC}$ calculation requires more than an order of magnitude more computing time and storage than a corresponding nonrelativistic or scalarrelativistic calculation. ${ }^{277}$ Meanwhile, the magnitude of the impact of scalar-relativistic effects on properties is usually substantially larger than that of spin-orbit effects. Therefore, a natural 
idea for a cost-effective treatment of relativistic effects at $\mathrm{CC}$ levels is to treat the larger but computationally less expensive scalar relativistic effects rigorously and then address spin-orbit effects by means of perturbation theory. In this context, the spin-free exact two-component theory in its one-electron variant (SFX2C-1e $)^{56,278,279}$ is highly recommended for a rigorous treatment of scalar-relativistic effects in routine chemical applications. The SFX2C-1e scheme performs an exact block-diagonalization of the spin-free version of the matrix representation of the Dirac Hamiltonian to decouple electronic and positronic degrees of freedom, and uses the electronic block of the resulting matrix representation of the Hamiltonian together with non-relativistic twoelectron integrals in the subsequent many-electron treatment. As scalar-relativistic corrections are dominated by one-electron contributions, ${ }^{278,280}$ the SFX2C-1e scheme is capable of providing an accurate treatment of scalar-relativistic effects on energies and properties. An SFX2C-1e calculation requires only additional manipulation of one-electron Hamiltonian integrals as compared to a non-relativistic calculation and thus essentially has the same computational cost, as mentioned above. The SFX2C-1e energy and analytic gradients ${ }^{56,281}$ are available in the released version of CFOUR. SFX2C-1e calculations of energies and first-order properties and geometry optimizations can conveniently be carried out. That is, the same input file used for the corresponding non-relativistic calculation needs only an instruction that the SFX2C-1e scheme is to be used (RELATIVISTIC=X2C1E), and then an appropriate basis set (recontracted for the SFX2C-1e scheme) needs to be selected. Table IV summarizes geometrical parameters for goldcontaining molecules computed at the non-relativistic and SFX2C-1e CCSD(T) levels. These SFX2C-1e CCSD(T) calculations have essentially identical computational cost as the corresponding non-relativistic ones; scalar-relativistic effects are obtained for free. In this demonstration, the availability of analytic gradients and the efficiency of the SFX2C-1e scheme allows a quick prediction for the geometry of an unknown gold-containing species $\left(\mathrm{AuCH}_{3}\right)$ with reasonably good accuracy, with one optimization cycle (one gradient calculation) taking only around $15 \mathrm{~min}$ utes using a single cpu [Intel(R) Xeon(R) CPU E5-2698v3@2.30GHz CPU] and 4 GB memory. More rigorous treatment of scalar-relativistic effects using the spin-free Dirac-Coulomb (SFDC) approach $^{282}$ or SFX2C in its mean-field variant (SFX2C-mf) ${ }^{283}$ have also been implemented in CFOUR. The SFDC approach features a spin separation in the four-component framework and is perhaps the most rigorous treatment of scalar-relativistic effects. SFDC is available in the released version of CFOUR for calculations of energies and first-order electrical properties (RELATIVISTIC=SFREE). ${ }^{55}$ The SFX2C-mf scheme recently implemented ${ }^{284}$ in CFOUR per- 
TABLE V. Geometrical parameters of $\mathrm{AuF}, \mathrm{AuCN}$, and $\mathrm{AuCH}_{3}$ computed at the non-relativistic and SFX2C-1e-CCSD(T) levels (bond lengths in $\AA$ and bond angle in degree). 1s electrons of C, N, and F as well as 1s, 2s, 2p, 3s, 3p, 3d, 4s, 4p, and 4d electrons of gold have been kept frozen in CC treatment. The ANO basis sets of triple-zeta quality used here have been obtained by recontracting ANO-RCC primitive sets $^{285,286}$ using non-relativistic and SFX2C-1e CCSD atomic densities, and can be found at www.cfour.de.

\begin{tabular}{ccccc}
\hline \hline & & Nonrelativistic & SFX2C-1e & Experiment \\
\hline \multirow{2}{*}{$\mathrm{AuF}$} & $\mathrm{R}(\mathrm{Au}-\mathrm{F})$ & 2.094 & 1.921 & 1.918 \\
$\mathrm{AuCN}$ & $\mathrm{R}(\mathrm{Au}-\mathrm{C})$ & 2.151 & 1.902 & 1.912 \\
& $\mathrm{R}(\mathrm{C}-\mathrm{N})$ & 1.171 & 1.168 & 1.159 \\
$\mathrm{AuCH}_{3}$ & $\mathrm{R}(\mathrm{Au}-\mathrm{C})$ & 2.207 & 1.984 & $/$ \\
& $\mathrm{R}(\mathrm{C}-\mathrm{H})$ & 1.088 & 1.088 & $/$ \\
& $\angle(\mathrm{Au}-\mathrm{C}-\mathrm{H})$ & 107.7 & 107.3 & $/$ \\
\hline \hline
\end{tabular}

forms the block-diagonalization at the HF level and will be available in the next released version.

Perturbative treatment of spin-orbit effects can be obtained using either the SFDC or the SFX2C-1e scheme as the zeroth-order treatment. ${ }^{287-289}$ For the latter, the corresponding spinorbit integrals are defined as first derivatives of SFX2C-1e Hamiltonian integrals, thereby treating spin-orbit integrals in the four-component formulation as the perturbation and using the analytic SFX2C-1e derivative technique. In this way, scalar-relativistic effects on spin-orbit integrals, which represent the coupling between scalar relativistic effects and spin-orbit coupling, have been taken into account. This greatly extends the applicability of the perturbative treatment of spin-orbit coupling in CFOUR to molecules containing heavy elements. Two-electron spin-orbit contributions can be taken into account using the molecular mean-field (MMF) or the atomic mean-field (AMF) spin-orbit approach. ${ }^{288,290,291}$ The resulting effective one-electron spin-orbit integrals can be contracted with one-electron transition density matrices to obtain spin-orbit matrix elements between two electronic states. The EOM-CCSD transition densities (also needed for the quasidiabatic couplings in Section IV. E) have been shown to provide accurate spin-orbit parameters $^{289,292,293}$ and are highly recommended for routine applications. Computed spin-orbit splittings of representative ${ }^{2} \Pi$ states using MMF and AMF spin-orbit integrals within the SFX2C- 
TABLE VI. Spin-orbit splittings (in $\mathrm{cm}^{-1}$ ) of ${ }^{2} \Pi$ radicals calculated at the SFX2C-1e-EOM-CCSD level using uncontracted ANO-RCC basis sets. "MMF" and "AMF" refer to molecular mean field and atomic mean field, respectively. The experimental values are given as compiled in Ref. 289.

\begin{tabular}{cccc}
\hline \hline & MMF & AMF & Experiment \\
\hline OH & 135.2 & 132.7 & 139 \\
SH & 369.5 & 369.3 & 377 \\
SeH & 1701.2 & 1700.9 & 1763 \\
TeH & 3675.3 & 3675.1 & 3816 \\
FO & 195.0 & 193.8 & 197 \\
ClO & 319.6 & 316.9 & 322 \\
BrO & 985.2 & 984.5 & 975 \\
IO & 2126.3 & 2124.0 & 2091 \\
\hline \hline
\end{tabular}

1e scheme at the EOM-CCSD level are summarized in Table V. The computed splittings compare very well with experimental values, with the biggest discrepancy being about $4 \%$ in the case of TeH. SFX2C-1e EOM-CCSD calculation of spin-orbit coupling matrix elements will be available in the next release of CFOUR.

CFOUR has also included options for non-perturbative treatment of spin-orbit coupling to obtain benchmark results or for studying heavy elements such as those in $6 \mathrm{p}$ or $7 \mathrm{p}$ blocks, for which these effects are too large to be handled using a perturbative treatment. The released version of CFOUR provides a spin-orbit CCSD(T) scheme for closed-shell systems. ${ }^{294}$ In this scheme a HF calculation using scalar-relativistic effective core potentials (ECP) is first performed to obtain orbitals. A corresponding ECP spin-orbit term is then included to augment the Fock matrix in subsequent $\mathrm{CC}$ calculations. Analytic first and second derivatives are available for this scheme in the released version of CFOUR. ${ }^{294-296}$ Recent developments along this line include EOMEE, EOMEA-, and EOMIP-CCSD methods. ${ }^{297-299}$ More recently, an X2C ${ }^{300-305}$ AMF approach has been developed for the non-perturbative treatment of spin-orbit coupling. ${ }^{59}$ Based on this approach, coupled-cluster methods [CCSD(T), EOM-CCSD, and EOM-CCSD(T)(a)*)] with spinorbit coupling included at the orbital level have been implemented. ${ }^{60,221,306}$ The focus of these 
studies is on efficient implementation using atomic orbital based algorithms and rigorous treatment of spin-orbit coupling in $\mathrm{X} 2 \mathrm{C}$. Users requesting more information about these relativistic methods in CFOUR are encouraged to make inquiries on the CFOUR mailing list (see Appendix 2).

\section{Multireference coupled-cluster methods}

The treatment of quasidegenerate systems with chemical accuracy is one of the most intriguing problems of electronic-structure theory. Although certain patterns of quasidegeneracy can be treated by means of EOM-CC methods (see Section IIC) or in terms of generalized singlereference CC methods, ${ }^{307,308}$ all these methods are subject to limitations, in particular a bias towards the selected reference determinant. The development of genuine multireference CC (MRCC) methods therefore remains an important goal of CC theory.

Much effort has been devoted to generalize the $\mathrm{CC}$ ansatz to the multireference domain, but this has turned out to be not straightforward: Many MRCC methods have been suggested and successfully applied to actual chemical problems, but a theory as elegant and robust as singlereference CC theory discussed in Section II A has yet to emerge. Comprehensive overviews of the field are provided, for example, in Refs. 61,309.

The development of MRCC theory in CFOUR has concentrated on the method suggested by Mukherjee and co-workers (Mk-MRCC). ${ }^{310,311}$ This is a state-specific MRCC variant relying on the Jeziorski-Monkhorst ansatz, ${ }^{312}$

$$
\left|\Psi^{\alpha}\right\rangle=\sum_{\mu}^{d} \exp \left(T_{\mu}\right)\left|\Phi_{\mu}\right\rangle c_{\mu}^{\alpha} .
$$

The reference determinants $\Phi_{\mu}$ differ in the occupation of the active orbitals; they form a model space of dimension $d$, their weighting coefficients $c_{\mu}^{\alpha}$ are optimized for a particular target state $\alpha$. The cluster operators $T_{\mu}$ are specific to reference $\Phi_{\mu}$ and can be partitioned into excitation classes in analogy to Eq. (2). So-called internal excitations that map $\Phi_{\mu}$ onto another reference determinant $\Phi_{v}$ need to be excluded from $T_{\mu}$. The energy $E^{\alpha}$ and the coefficients $c_{\mu}^{\alpha}$ are obtained as eigenvalue and eigenvector of an effective Hamiltonian, whose elements are $H_{\mu \nu}^{\text {eff }}=\left\langle\Phi_{\mu}\left|\exp \left(-T_{v}\right) H \exp \left(T_{v}\right)\right| \Phi_{v}\right\rangle$. The amplitude equations take on the form

$$
\begin{gathered}
\left\langle\Phi_{P}(\mu)\left|\exp \left(-T_{\mu}\right) H \exp \left(T_{\mu}\right)\right| \Phi_{\mu}\right\rangle c_{\mu}^{\alpha} \\
+\sum_{v \neq \mu}\left\langle\Phi_{P}(\mu)\left|\exp \left(-T_{\mu}\right) \exp \left(T_{v}\right)\right| \Phi_{\mu}\right\rangle H_{\mu \nu}^{\mathrm{eff}} c_{v}^{\alpha}=0 .
\end{gathered}
$$


with $\Phi_{P}(\mu)$ as excitation manifold specific to reference $\Phi_{\mu}$. The first term of Eq. (20) can be interpreted as a generalization of Eq. (4) whereas the second term couples the amplitude equations for different cluster operators $T_{\mu}, T_{\nu}$. In practice, the cluster operators are usually truncated in analogy to the single-reference case giving rise to the Mk-MRCCSD, ${ }^{310,311,313}$ Mk-MRCCSDT, 314 etc. models.

Distinct advantages of Mk-MRCC theory include rigorous size-extensivity, ${ }^{311}$ the unbiased treatment of all references $\Phi_{\mu}$ in the model space, ${ }^{61}$ and conceptual simplicity resulting in relatively simple working equations. ${ }^{313}$ However, all truncated MRCC methods based on Eq. (19) are not invariant with respect to rotations among the active orbitals ${ }^{61,315}$ and it has also been shown that the computation of excitation energies and frequency-dependent properties by means of linear-response theory is problematic with Mk-MRCC methods because the pole structure of the linear-response function is flawed. ${ }^{316,317}$ Furthermore, the number of amplitudes to be determined is proportional to the size of the model space. As a consequence, the computational cost scales with system size as $d$ times that of the corresponding single-reference model, that is, $d \cdot M^{6}$ for Mk-MRCCSD,$d \cdot M^{8}$ for Mk-MRCCSDT and so forth, making Mk-MRCC impractical for large model spaces. ${ }^{61}$

CFOUR offers efficient Mk-MRCCSD ${ }^{318}$ and Mk-MRCCSDT ${ }^{66}$ implementations for a model space of two closed-shell determinants. An implementation of Mk-MRCC for arbitrary excitation levels and model spaces has been presented elsewhere. ${ }^{319}$ The CFOUR implementation is adequate for biradical species and single-bond breaking and, therefore, applicable to many multireference cases. In these calculations, orbitals can be taken either from an HF or a two-configurational SCF calculation. The application of Mk-MRCCSDT to larger molecules is greatly facilitated by means of parallelization; that is, computing the triple amplitudes and their contributions to the singles and doubles residuals in a distributed manner. Mk-MRCCSDT computations using well over 200 basis functions have been carried out with CFOUR. ${ }^{66}$ A non-iterative treatment of triple excitations, termed Mk-MRCCSD(T), has also been implemented into CFOUR for model spaces of two closed-shell determinants. ${ }^{320}$ The treatment of open-shell states is possible at the Mk-MRCCSD level using a model space of two open-shell determinants and orbitals from a lowspin ROHF calculation. ${ }^{321}$ The case of a full model space of two electrons distributed among two orbitals (comprising four reference determinants) can also be treated at the Mk-MRCCSD level.

Larger model spaces are required if more than two orbitals are (quasi-)degenerate. Examples include the breaking of double and triple bonds as well as many transition-metal compounds. ${ }^{61}$ 

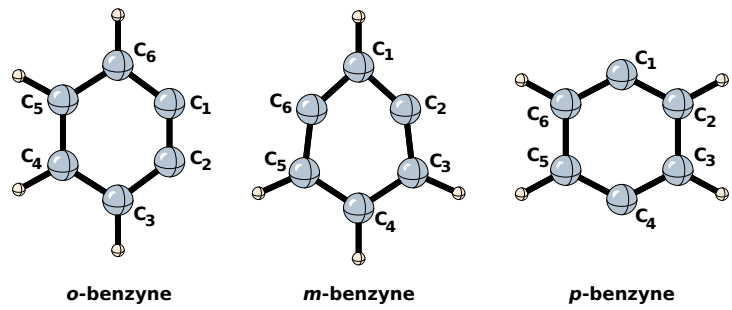

FIG. 3. Optimized structures of the ground states of the three isomers of benzyne computed at the MkMRCCSD/cc-pCVTZ level of theory. Taken from Ref. 321

Such cases can be treated by means of internally contracted (ic)-MRCC methods ${ }^{73,322-326}$ implemented in the GECCO program ${ }^{73}$ that has been interfaced to CFOUR. ${ }^{74}$ In ic-MRCC theory, a single cluster operator acts on a multideterminantal reference. ic-MRCC methods maintain full orbital invariance and size extensivity, their computational cost is roughly comparable to that of the corresponding single-reference method. ${ }^{61,73}$ However, the working equations are considerably more complicated mandating automated implementation techniques. ${ }^{73}$

As a unique feature, CFOUR offers efficient implementations of analytic gradients at the MkMRCCSD $^{318,327}$ and Mk-MRCCSDT ${ }^{328}$ levels of theory. The theory is formulated starting from a Lagrangian functional in analogy to single-reference CC gradient theory (see Section II B). The Mk-MRCC gradient can be written as ${ }^{318}$

$$
\frac{d E}{d x}=\sum_{\mu} \bar{c}_{\mu} c_{\mu}\left\langle\Phi_{\mu}\left|\left(1+\Lambda_{\mu}\right) \exp \left(-T_{\mu}\right) \frac{d H}{d x} \exp \left(T_{\mu}\right)\right| \Phi_{\mu}\right\rangle
$$

with $\Lambda_{\mu}$ as analog to the $\Lambda$ operator from Eqs. (6) and (7) and $\bar{c}_{\mu}$ as additional Lagrange multipliers. Eq. (21) is evaluated based on density matrices; the relevant details are discussed in Ref. 318. Besides enabling geometry optimizations of polyatomic molecules, ${ }^{318,321,327}$ analytic gradients also provide convenient access to harmonic vibrational frequencies through numerical differentiation.

To give an example of Mk-MRCC geometry optimizations, Table VII shows selected structural parameters for the ground states of the three isomers of benzyne depicted in Figure 3. The electronic structure of these biradicals can be understood qualitatively in terms of two frontier MOs that are a bonding and an antibonding combination of the atomic orbitals hosting the radical electrons. The wave functions are dominated by two closed-shell determinants whose weights computed with Mk-MRCCSD are also included in Table VII, this illustrates that the multireference character increases from the $o$ - to the $m$ - to the $p$-isomer. Owing to the shape of the frontier 
TABLE VII. $\mathrm{C}_{1}-\mathrm{C}_{2}$ distances for $o$-benzyne, $\mathrm{C}_{2}-\mathrm{C}_{6}$ distances for $m$-benzyne, and $\mathrm{C}_{1}-\mathrm{C}_{4}$ distances for p-benzyne in $\AA$ computed at the Mk-MRCCSD, CCSD, and CCSD(T) levels of theory using the cc-pCVTZ basis set. The weights of the reference determinants (see Eq. (19)) are also shown. For further details, see Ref. 321.

\begin{tabular}{|c|c|c|c|}
\hline & $o$-benzyne & $m$-benzyne & p-benzyne \\
\hline $\mathrm{R}$ [CCSD] & 1.2436 & $ـ^{a}$ & 2.7071 \\
\hline $\mathrm{R}[\mathrm{CCSD}(\mathrm{T})]$ & 1.2567 & 2.0432 & 2.7183 \\
\hline R [Mk-MRCCSD] & 1.2505 & 2.0141 & 2.6865 \\
\hline$\left|c_{1}\right|^{2}[\mathrm{Mk}-\mathrm{MRCCSD}]$ & 0.942 & 0.921 & 0.724 \\
\hline$\left|c_{2}\right|^{2}[\mathrm{Mk}-\mathrm{MRCCSD}]$ & 0.058 & 0.079 & 0.276 \\
\hline
\end{tabular}

${ }^{\text {a }}$ CCSD calculations for $m$-benzyne favor a bicyclic structure without multireference character.

TABLE VIII. Spin-orbit splittings in $\mathrm{cm}^{-1}$ calculated at the Mk-MRCCSD/cc-pVQZ ${ }^{a}$ level of theory using the spin-orbit mean-field approximation. Experimental data are also given. For further details, see Ref. 329.

\begin{tabular}{crr}
\hline \hline Molecule Mk-MRCCSD & \multicolumn{1}{c}{ Expt. } \\
\hline $\mathrm{OH}$ & 135.1 & 139.2 \\
$\mathrm{SH}$ & 375.2 & 377.0 \\
$\mathrm{SeH}$ & 1707.9 & 1763.3 \\
$\mathrm{NCS}$ & 360.8 & 325.3 \\
\hline \hline
\end{tabular}

${ }^{a}$ g-functions have been omitted.

MOs, the distance between the two radical centers provides a measure for the influence of the two reference determinants on the molecular equilibrium structures. ${ }^{318,327}$ Table VII illustrates good agreement between CCSD and Mk-MRCCSD for $o$-benzyne whereas larger deviations are observed for the other two isomers with stronger multireference character.

In addition to geometrical derivatives, CFOUR can compute spin-orbit (SO) splittings for ${ }^{2} \Pi$ states based on degenerate perturbation theory as a first-order property at the Mk-MRCCSD level of theory. ${ }^{329}$ This constitutes an alternative to the computation of these quantities by means of EOM-CC theory (see Table VI) and is also helpful for the theoretical analysis of MRCC models relying on Eq. (19). For such methods the symmetry properties of the SO operator allow for a de- 
composition of the SO splitting expression into two terms: A similarity-transformed SO operator times a coupling term intimately related to the coupling term from Eq. (20). It has been argued ${ }^{329}$ that SO splittings provide a quality measure for this coupling term. As a numerical example, Table VIII shows SO splittings for the ${ }^{2} \Pi$ states of a few diatomic and triatomic molecules.

\section{E. Vibronic Hamiltonians and Electronic Spectroscopy}

A relatively common application of quantum chemistry is to electronic spectroscopy, the full understanding of which requires knowledge of electronic, vibrational and (sometimes) rotational energy levels. While many electronic transitions, photoionization and electron detachment processes are well-described by the Franck-Condon approximation, this is not always the case. A standard approach for treating these difficult cases - which involve Herzberg-Teller or true nonadiabatic effects - is to construct a molecular Hamiltonian in an electronic basis that does not consist of the usual adiabatic states typically obtained in quantum chemical calculations. A convenient framework for such an analysis was devised by Köppel, Domcke, and Cederbaum (KDC), 330 who applied it long ago with great success to a number of photoelectron spectra in which ionization to the lowest-lying ionic states was inadequately treated by the Franck-Condon picture. ${ }^{331}$

In such calculations, the molecular Hamiltonian is written in a basis of "quasidiabatic" electronic states which, by construction, vary smoothly and slowly as the nuclei are displaced. This assumption motivates the form of the (diagonal) kinetic energy operator, but means that the potential energy (the usual electronic Hamiltonian) is not diagonal. For a two state problem, this model vibronic Hamiltonian takes the form

$$
H_{K D C}=T+V=\left(\begin{array}{cc}
T_{a} & 0 \\
0 & T_{b}
\end{array}\right)+\left(\begin{array}{cc}
V_{a a} & V_{a b} \\
V_{a b} & V_{b b}
\end{array}\right),
$$

which is usually projected onto a vibrational basis and then diagonalized to compute the spectrum and intensities. A particularly simple form is given by the so-called linear vibronic coupling model (LVC), viz.

$$
V_{L V C}=\left(\begin{array}{cc}
\sum_{s} \kappa_{s}^{a} q_{s}+\frac{1}{2} \sum_{k} \omega_{k} q_{k}^{2} & \sum_{c} \lambda_{c} q_{c} \\
\sum_{c} \lambda_{c} q_{c} & \Delta_{a b}+\sum_{s} \kappa_{s}^{b} q_{s}+\frac{1}{2} \sum_{k} \omega_{k} q_{k}^{2}
\end{array}\right)
$$

which in this form is applicable to the pseudo-Jahn-Teller case, where interaction between two (generally quite proximate) non-degenerate states are important. 
Treatments of electronic spectra with the KDC model can involve an arbitrarily large number of electronic states (for example, a proper treatment of the $\mathrm{NO}_{3}$ radical requires at least five states, ${ }^{332}$ ) and going beyond the LVC is sometimes necessary to obtain qualitative understanding, and always necessary for quantitative agreement with measured spectra. Moreover, true Jahn-Teller cases (interaction between degenerate states) can also be treated with largely the same framework. Nevertheless, the very simple non-degenerate two-state LVC model is an appropriate example to explain what tools are available in CFOUR for such calculations. Details for how more elaborate calculations are done can be found elsewhere. ${ }^{333,334}$

The form of the LVC Hamiltonian above involves a choice of normal coordinates $(q)$, the gap between the electronic states at the coordinate origin ( $\Delta_{a b}$, assumed to be positive below), linear diagonal terms with coefficients $\kappa_{s}$ that correspond to gradients along totally symmetric coordinates $\left(q_{s}\right)$ on the adiabatic potential energy surface, quadratic force constants for all modes on the diagonal (in the LVC model, these are assumed to be equal to the reference state for which the normal coordinates are calculated), and - critically - an off-diagonal coupling in which modes $q_{c}$ of a certain symmetry (for example the asymmetric $b_{2}$ NO stretching mode if the two states are the $\tilde{X}^{2} A_{1}$ and $\tilde{A}^{2} B_{2}$ states of $\mathrm{NO}_{2}$ ) carry quasidiabatic coupling constants $\lambda_{c}$. Without sacrificing simplicity, a useful extension of the LVC model is to maintain the assumption of linear off-diagonal coupling, but to allow the quadratic force constants to relax from those of the reference state, which leads to

$$
V=\left(\begin{array}{cc}
\sum_{s} \kappa_{s}^{a} q_{s}+\frac{1}{2} \sum_{k l} g_{k l}^{a} q_{k} q_{l} & \sum_{c} \lambda_{c} q_{c} \\
\sum_{c} \lambda_{c} q_{c} & \Delta_{a b}+\sum_{s} \kappa_{s}^{b} q_{s}+\frac{1}{2} \sum_{k l} g_{k l}^{b} q_{k} q_{l}
\end{array}\right)
$$

The computation of all parameters begins with the determination of a set of normal coordinates, which usually are those of the same molecule in a different (reference) electronic state, with the absorbing state in the spectroscopic experiment being the most logical choice. For example, to study photodetachment of $\mathrm{NO}_{2}^{-}$, one would choose the anion. To do an LVC calculation, the first and second derivatives of the energies at the origin of the coordinate system (i.e. the geometry of $\mathrm{NO}_{2}^{-}$) are evaluated using the derivative techniques in CFOUR and then transformed into the normal coordinates. CFOUR contains a module called xquadmodel for effecting this transformation. The quasidiabatic coupling constants $\left(\lambda_{c}\right)$ above are evaluated according to a diabatization 
scheme based on EOM-CC theory that is described in detail elsewhere, ${ }^{50}$ and their evaluation is based on an algorithm that is quite similar to that for adiabatic EOM-CC gradients. However, transition one- and two-electron densities are used in this case, and there are additional minor modifications necessitated by the different physical situation under consideration. It is important to note that these are not "non-adiabatic couplings" (which are off-diagonal terms in the kinetic energy in the adiabatic basis rather than off-diagonal terms in the potential energy in the quasidiabatic basis), but are intimately related to them, as is discussed in Refs. ${ }^{51,335,336}$. In any event, once the quasidiabatic couplings are calculated, the force constants of the coupling modes appearing in the diagonal blocks of the potential are "diabatized" via

$$
\begin{aligned}
& g_{c c^{\prime}}^{a}=\left(f_{c c^{\prime}}^{A}\right)_{\text {adiabatic }}+\frac{2 \lambda_{c} \lambda_{c}^{\prime}}{\Delta_{a b}} \\
& g_{c c^{\prime}}^{b}=\left(f_{c c^{\prime}}^{A}\right)_{\text {adiabatic }}-\frac{2 \lambda_{c} \lambda_{c}^{\prime}}{\Delta_{a b}},
\end{aligned}
$$

where the $f_{c c^{\prime}}$ are the quadratic force constants on the adiabatic potential surfaces. For coefficients $g_{k l}$ where $q_{k}$ and $q_{l}$ do not couple the states, these are simply equal to the corresponding adiabatic force constants on the two surfaces. Together with the trivially-calculated $\Delta_{a b}$, all parameters for the Hamiltonian are now available, and the xsim module of CFOUR can then carry out the spectral simulation.

It should be emphasized that the crucial coupling of states that characterizes these situations makes special demands on the quantum-chemical method. Approaches appropriate for the parametrization are many, but generally do not include ground-state single determinant MBPT and CC methods. It has been recognized that EOM-CC methods are ideally suited for problems of this sort, ${ }^{75,337}$ and are recommended for applications. For the example above (the photodetachment spectrum of $\mathrm{NO}_{2}^{-}$), EOMIP-CC is the most appropriate method, and the gradients available in CFOUR (together with the quasidiabatic coupling calculation) greatly facilitate the calculations that need to be done to construct the Hamiltonian. Quasidiabatic couplings can currently be routinely evaluated with EOMEE-CCSD only, with the continuum orbital approach recommended for EOMIP-CCSD and EOMEA-CCSD calculations.

Documentation about vibronic Hamiltonian construction and diagonalization calculations is spotty, and the process of carrying out these calculations (apart from the simplest LVC treatment) is slightly arduous and tedious. In general, the procedure involves three phases. In the first the reference state (that which is used to define normal coordinates, and is usually the absorbing state in the experiment) is characterized by means of geometry optimization and second derivative cal- 
culations. Then, the first and second derivatives are calculated for the final states, and transformed to the reference state normal coordinates. Beyond this, the quasidiabatic couplings are calculated and similarly transformed. For an LVC (or slightly elaborated LVC calculation, as is demonstrated in the following paragraph), these are the three required phases of quantum chemistry calculation. Any investigators that require assistance with such calculations, or intend to explore more elaborate vibronic coupling models with CFOUR are encouraged to seek advice and assistance from the CFOUR mailing list (see Appendix 2). This will also permit them to be directed to the tools that have been created by the authors to facilitate this particular type of spectroscopic application, and instructed in their use.

Parameters, and a simulation, are shown for the $\mathrm{NO}_{2}^{-}$photodetachment spectrum in Table IX and Fig. 4, respectively, where the latter may be compared to the laboratory spectrum. The calculations were done at the frozen-core CCSD/cc-pVDZ level of theory (the anion is treated with simple single-reference CCSD and the $\tilde{X}^{2} A_{1}$ and $\tilde{A}^{2} B_{2}$ states of $\mathrm{NO}_{2}$ are treated with EOMIPCCSD), and the simulated spectrum shows indeed the power of the LVC model for capturing the salient qualitative features of electronic spectra. It is an entirely straightforward matter to do this parameterization and spectroscopic calculation with CFOUR; the entire procedure can easily be done in a few hours of work.

Finally, for simpler electronic spectra in which interactions between electronic states can be neglected, CFOUR has a highly-efficient Franck-Condon program xfc_squared, ${ }^{339}$ and clear documentation for running it is available on the CFOUR website (see Appendix 1).

\section{F. Automatized composite schemes and basis-set extrapolations}

Additivity schemes and basis-set extrapolation 340,341 are nowadays popular tools to minimize both basis-set truncation errors and correlation errors and to provide high-accuracy quantumchemical results. ${ }^{233,236,342,343}$ While these schemes are easily handled (with a calculator or a spreadsheet) when focusing on energies, their application is much more cumbersome in the context of geometry optimization or the computation of other properties. CFOUR offers here an automatized scheme, ${ }^{68,344}$ which within a geometry optimization sets up and runs all individual computations that are needed, gathers the result, and computes the total energy and gradient.

As input for computations involving basis-set extrapolation as well as composite schemes, CFOUR requires (a) the property to be computed (energy, geometry, or harmonic frequencies), 
TABLE IX. Parameters of the LVC Hamiltonian describing the photodetachment spectrum of $\mathrm{NO}_{2}^{-}$, obtained at the fc-(EOMIP)-CCSD/cc-pVDZ level of theory. The geometry of the anion is $\mathrm{R}(\mathrm{N}-\mathrm{O})=1.262$ $\AA, \theta=116.44^{\circ}$, and the anion harmonic frequencies are $\omega_{1}=1356.7 \mathrm{~cm}^{-1} ; \omega_{2}=794.6 \mathrm{~cm}^{-1}$; and $\omega_{3}=$ $1322.7 \mathrm{~cm}^{-1}$. The first two modes have $a_{1}$ symmetry, and the third mode (which couples the two states) has $b_{2}$ symmetry. All parameters are in $\mathrm{cm}^{-1}$.

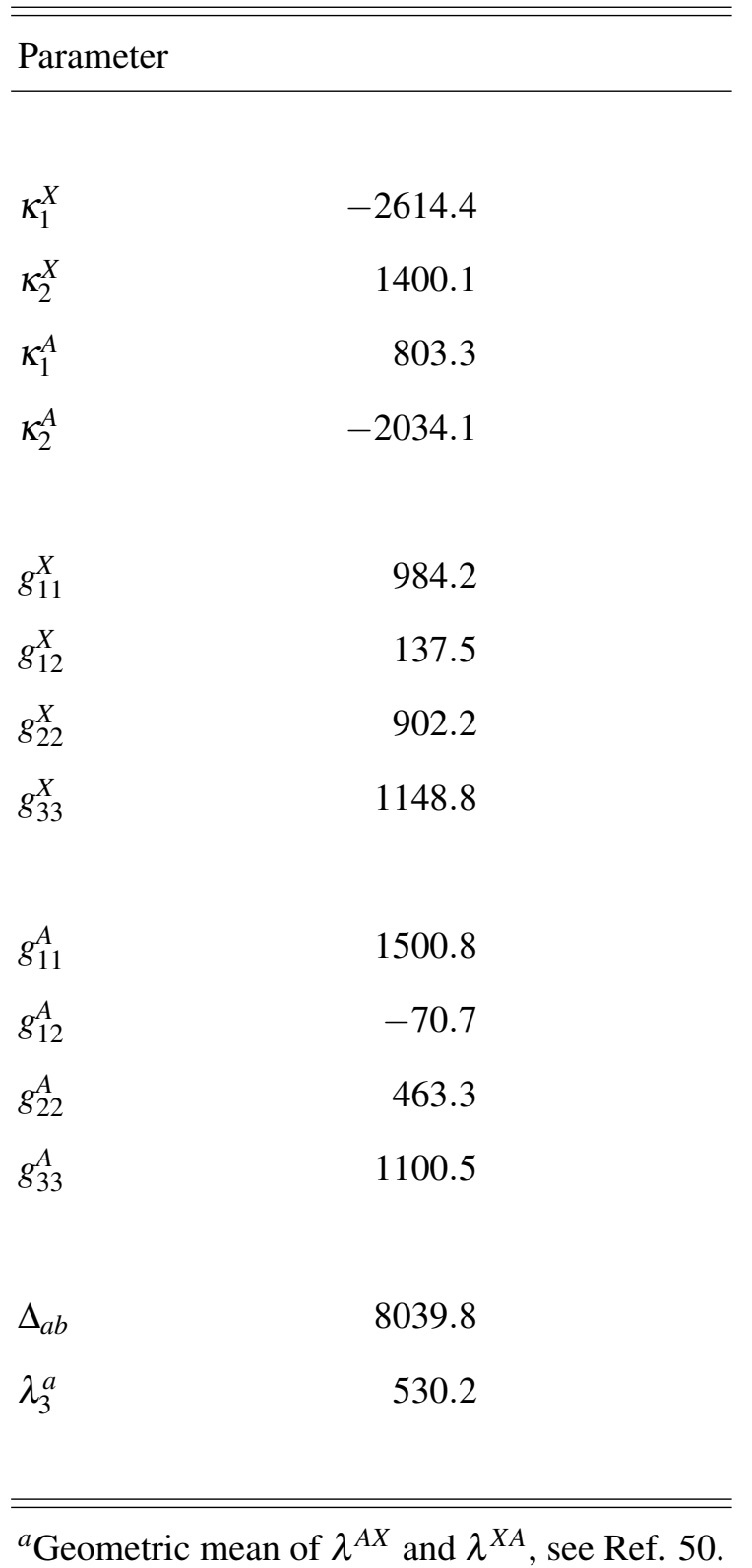

b) information concerning the basis sets used in the extrapolation (three basis sets from one of the correlation-consistent hierarchies of basis sets ${ }^{266,345}$ are required for the extrapolation at the HF level; ${ }^{340}$ two sets are needed for the extrapolation at the correlated level ${ }^{341}$ ), c) information 


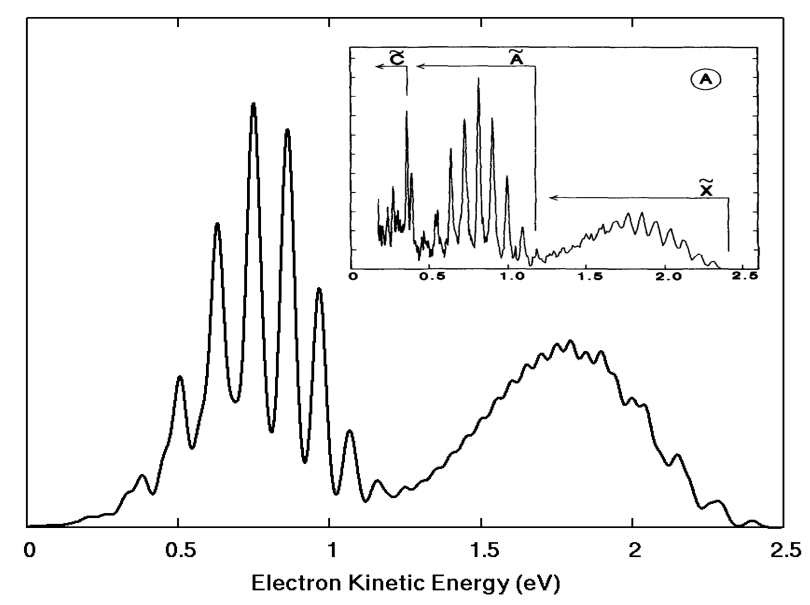

FIG. 4. Simulation of the $266 \mathrm{~nm}(4.66 \mathrm{eV})$ photodetachment spectrum of the $\mathrm{NO}_{2}$ anion, using the parameters in Table IX and calculated with the xsim module of CFOUR. The vertical energies have been adjusted by $+0.2 \mathrm{eV}$, so as to have the origin (peak at highest eKE) approximately coincide with that in the laboratory measurement of Ref. 338 (inset). This shift accounts for an underestimation of the electron affinity at the EOMIP-CCSD level of theory with the aug-cc-pVDZ basis set. In the simulation, each state in the stick spectrum has been convoluted with Gaussians having a width of $0.05 \mathrm{eV}$. Note that the experimental spectrum reveals a higher excited state of $\mathrm{NO}_{2}$ (at low electron kinetic energy), which was not included in the simulation. The two-state Hamiltonian was projected onto a vibrational basis comprising 25 functions per mode and diagonalized using 1000 Lanczos recursions. Transition moments for the two ionization processes are assumed to be equal. The inset was reproduced from Ref. 338 with the permission of AIP Publishing.

about the additional corrections to be applied, i.e., those from CCSDT, CCSDTQ, or all-electron $\operatorname{CCSD}(\mathrm{T})$ computations, and d) keywords for the individual calculations to be performed. Detailed information about the input can be found on the CFOUR website (see Appendix 1).

It should be pointed out that the computation of equilibrium geometries and harmonic vibrational frequencies in this way provides results that are consistent with the potential energy surface defined by the extrapolated energy. This is accomplished by using, for the gradient or the corresponding second derivatives, expressions that are derived from the original extrapolated energy by means of straightforward differentiation. ${ }^{68}$

To give an example, Figure 5 compares the equilibrium geometry of cyclic $\mathrm{SiS}_{2}$ obtained at the 
$\mathrm{HF} / \infty \mathrm{Z}+\mathrm{CCSD}(\mathrm{T}) / \infty \mathrm{Z}+\Delta \mathrm{T} / \mathrm{cc}-\mathrm{pVTZ}+\Delta \mathrm{Q} / \mathrm{cc}-\mathrm{pVDZ}+$ core/cc-pCV5Z level, at which computations using the cc-pVQZ, cc-pV5Z, and cc-pV6Z basis sets are used to estimate the HF limit and computations at cc-pV5Z and cc-pV6Z level to obtain the basis-set limit for the fc-CCSD(T) correlation energy. Additional contributions involve a correction obtained at the fc-CCSDT level (in comparison to fc-CCSD(T)) computed with the cc-pVTZ set, a correction obtained at the fc-CCSDTQ level (in comparison to fc-CCSDT) evaluated with cc-pVDZ, and a correction for core-correlation effects obtained at the $\operatorname{CCSD}(\mathrm{T}) / \mathrm{cc}-\mathrm{pCV} 5 \mathrm{Z}$ level (in comparison to frozen-core $\operatorname{CCSD}(\mathrm{T}))$. The experimental equilibrium geometry ${ }^{346}$ has been obtained from rotational constants determined for three isotopologues of cyclic $\mathrm{SiS}_{2}$. These rotational constants have been adjusted using vibrational corrections computed at the CCSD(T)/cc-pCVTZ level using VPT2. ${ }^{67}$ Concerning harmonic vibrational frequencies, the extrapolation scheme yields 1647, 3836, and $3947 \mathrm{~cm}^{-1}$ which can be compared to the experimental inferred values of $1648.5,3832.2$, and $3942.5 \mathrm{~cm}^{-1} .347$

Statistical analyses of the performance of these extrapolation schemes can be found in Ref. 68 for equilibrium geometries and in Ref. 35 for rotational constants derived from computed geometries after taking account of vibrational corrections. In passing, we note that the extrapolation scheme can be further augmented by scalar-relativistic corrections computed either at the DPT2 level or using the $\mathrm{X} 2 \mathrm{C}$ scheme.

\section{G. Analytic calculation of the Diagonal Born Oppenheimer Correction (DBOC)}

The Born-Oppenheimer approximation ${ }^{348}$ (BOA) is a fundamental assumption used in the description of molecules: not only are quantum-chemical calculations mostly based on it, but also chemical intuition relies on the notion of potential energy surfaces defined by the BOA. It is a quite good approximation and as cause for its breakdown typically (near-)degeneracy of coupled electronic states ${ }^{349}$ are mentioned. The first-order correction to the $\mathrm{BOA}^{350}$ is, however, not related explicitly to other electronic states, ${ }^{351}$ it comes from the (parametric) dependence of the electronic wave function on the nuclear coordinates, which results in a nonzero expectation value of the nuclear kinetic energy operator over the electronic wavefunction:

$$
\Delta E_{\mathrm{DBOC}}(\mathbf{R})=\int \mathrm{d} \mathbf{r} \Psi^{*}(\mathbf{r} ; \mathbf{R}) T_{N}(\mathbf{R}) \Psi(\mathbf{r} ; \mathbf{R})
$$

with $\Psi$ as the normalized electronic wavefunction obtained within the BOA and $T_{N}$ as the nuclear kinetic energy operator. In Eq. (27), the electronic coordinates are collectively denoted by $\mathbf{r}$, while 


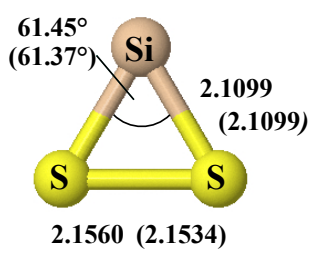

FIG. 5. Computed and semi-experimental equilibrium structure of cyclic $\mathrm{SiS}_{2}$. The semi-experimental structure was obtained via a least-squares fit of the geometrical parameters of cyclic $\mathrm{SiS}_{2}$ to the experimentally determined rotational constants of three isotopologues, the theoretical one (in parentheses) was obtained via composite computations as described in the text. All distances are given in $\AA$ and all angles in degrees. For further details, see Ref. 346.

the nuclear coordinates are represented by $\mathbf{R}$. The integration in Eq. (27) is over electronic coordinates only, thus the so-called diagonal Born-Oppenheimer correction (DBOC) depends parametrically on the nuclear coordinates and represents a mass-dependent increment to the potential energy surface. Thus, with the DBOC included in the calculation, the adiabatic picture is kept $\mathrm{t}^{352}$ (the DBOC is sometimes also called the adiabatic correction), and the notion of potential energy surfaces is retained, although they now become mass-dependent. The DBOC is numerically small, but the high accuracy reached by electronic structure methods, as also discussed at several parts of this paper, sometimes necessitates its inclusion in the final energy.

Since the kinetic energy operator in Eq. (27) includes the second derivative with respect to nuclear coordinates $\left(R_{A i}\right)$, the key to the computation of the DBOC is the evaluation of the expectation value of the operator $\nabla_{R_{A i}}^{2}$ over the electronic wavefunction. ${ }^{353}$ Replacing this second derivative by first derivatives of both the right- and left-hand $\mathrm{CC}$ wavefunctions, we were able to formulate the DBOC at the general CI level. ${ }^{48}$ However, calculation of the DBOC from coupled-cluster electronic wave function is complicated by the biorthogonal approach with different right- and lefthand wavefunctions, ${ }^{151,187}$ especially by issues associated with normalization. These problems have been resolved in Ref. 48 and the DBOC expression could be formulated using derivatives of 
the cluster and $\Lambda$ operators, the antisymmetric CC derivative density matrix, as well as the oneand two-particle unrelaxed density matrices.

Evaluation of the DBOC formulae is possible with gradient and second derivative techniques available in CFOUR and MRCC: the derivative of the amplitudes and the $\Lambda$ parameters can be taken directly from analytic force constant calculations. The same also holds for the calculation of the unperturbed one- and two-particle density matrices. Two differences need to mentioned: a) for the DBOC, unrelaxed density matrices are required, while the relaxed density matrices are used for the force constants; b) translational invariance, which is exploited in force-constant calculations, cannot be used for the DBOC since derivatives according to all nuclear coordinates are required. The latter difference makes a slight increase in computational time, while the first one precludes the possibility of doing DBOC and force constant calculations at the same time. The dependence of the computational effort on the size of the system is the same as for the underlying CC model, but the loop over the complete set of nuclear coordinates introduces an additional factor of $3 N_{\text {atoms }}$ with $N_{\text {atoms }}$ being the number of atoms in the considered molecule. Thus, the calculation of the DBOC is rather expensive compared to a single-point energy evaluation, nevertheless it can always be routinely performed when harmonic frequencies and zero-point energy corrections to the energy can be calculated analytically.

Availability of the DBOC for CC (and CI) methods analytically in CFOUR is the same as that of the analytic second derivative as shown Table I. The only exceptions are non-iterative methods like $\operatorname{CCSD}(\mathrm{T})$, where, due to the lack of a well defined wave function, the DBOC cannot be expressed in the above formalism. For more details, see Ref. 48. We note that according to numerical tests, ${ }^{48}$ triples contributions are rather small even at the full CCSDT level; therefore a CCSD(T) type DBOC would not bring substantial improvement over CCSD.

To offer a reduced-cost alternative to $\mathrm{CC}$ methods, in Ref. 49 approximations to the above theory within many-body perturbation theory were presented. The first one, termed MP1, uses first-order amplitudes in the formula and its perhaps unusual name reflects the fact that, contrary to the total energy, there is a first-order correction to the DBOC even in the Møller-Plesset partitioning. MP1-level DBOC just requires the evaluation of first-order (MP2) double excitation amplitudes and their contraction with corresponding DBOC integrals, i.e. no significant additional cost compared to the HF-SCF evaluation of the DBOC (provided the CPHF equations are solved). The next level is MP2, which requires the knowledge of the first- and second-order single and double excitation amplitudes. Higher order formulas have not been worked out since the cost 


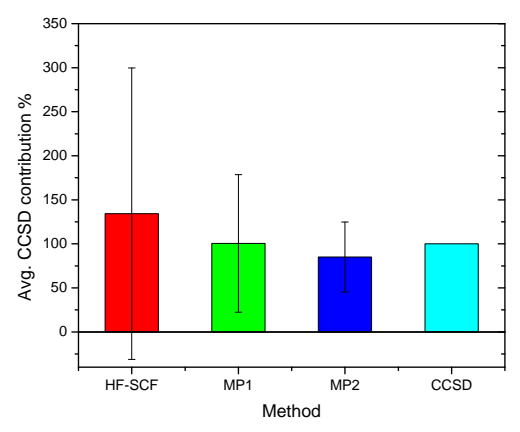

FIG. 6. Average DBOC contribution to atomization energies with respect to the CCSD value (in \%). Standard deviation is given as error bars. Data from Table $\mathrm{X}$ have been used, NO and OF excluded.

of their evaluation would be similar to CCSD.

The calculated DBOC has found most of its application in accurate prediction of thermochemical values, ${ }^{235,354-356}$ as well as in spectroscopy. ${ }^{357-364}$ To demonstrate its importance, the DBOC contributions to the atomization energies of selected small molecules are given in Table $\mathrm{X}$, as obtained by different methods. The table shows that the DBOC contribution can be as large as several tenth of $\mathrm{kJ} \mathrm{mol}^{-1}$, therefore non-negligible in certain applications. Indeed, as has been shown e.g. in Refs. 49 and 355, the DBOC contribution increases with the number hydrogen atoms, and its role can be even more important for larger molecules with many hydrogen atoms.

The importance of electron correlation and the accuracy of different methods is represented graphically in Figure 6. Here, the average DBOC contribution to atomization energies with respect to the CCSD value (100\%) is presented. One can conclude that a) correlation contribution is important, and its size is unpredictable (as shown by the large standard deviation of the SCF values); b) both MP1 and MP2 give good estimates with decreasing error bars.

\section{H. Core-level spectroscopy}

Core electron photoelectron and absorption spectra have served as useful tools for probing local chemical environments in molecules and solids. ${ }^{365,366}$ Recent developments of x-ray light sources have also led to a rapid growth in investigations of x-ray induced ultrafast dynamics. ${ }^{367,368}$ Accurate calculations of core ionization and excitation energies and of $\mathrm{x}$-ray absorption spectra are of significant interest and have been a longstanding challenge for quantum chemistry. ${ }^{369}$ Benefiting from available efficient implementation of EOM-CC methods, CFOUR offers EOM-CC 
TABLE X. DBOC contribution to atomization energies (in $\mathrm{kJ} \mathrm{mol}^{-1}$ ) of selected small systems calculated by different methods. The raw data are taken from Ref. 49 and obtained with the aug-cc-pCVQZ basis at $\operatorname{CCSD}(\mathrm{T}) / \mathrm{cc}-\mathrm{pVQZ}$ geometries.

\begin{tabular}{lcccc|lllll}
\hline \hline & SCF & MP1 & MP2 & CCSD & & SCF & MP1 & MP2 & CCSD \\
\hline $\mathrm{C}_{2} \mathrm{H}_{2}$ & 0.50 & 0.39 & 0.36 & 0.33 & $\mathrm{HCN}$ & 0.31 & 0.24 & 0.20 & 0.20 \\
$\mathrm{CCH}$ & 0.13 & 0.10 & 0.14 & 0.10 & $\mathrm{HCO}$ & -0.14 & -0.19 & -0.21 & -0.21 \\
$\mathrm{CF}$ & 0.03 & 0.01 & 0.01 & 0.01 & $\mathrm{HF}$ & 0.00 & 0.00 & 0.00 & 0.00 \\
$\mathrm{CH}$ & -0.35 & -0.41 & -0.42 & -0.44 & $\mathrm{HNO}-0.23$ & -0.31 & -0.36 & -0.40 \\
$\mathrm{CH}_{2}$ & 0.13 & 0.06 & 0.06 & 0.06 & $\mathrm{HO}_{2}$ & 0.03 & -0.01 & -0.03 & -0.04 \\
$\mathrm{CH}_{3}$ & 0.19 & 0.07 & 0.08 & 0.06 & $\mathrm{~N}_{2}$ & 0.08 & 0.04 & 0.01 & 0.03 \\
$\mathrm{CN}^{2}$ & -0.07 & -0.07 & -0.02 & 0.06 & $\mathrm{NH}$ & -0.21 & -0.24 & -0.24 & -0.24 \\
$\mathrm{CO}^{2}$ & 0.07 & 0.03 & 0.02 & 0.02 & $\mathrm{NH}$ & -0.05 & -0.12 & -0.12 & -0.14 \\
$\mathrm{CO}_{2}$ & 0.20 & 0.16 & 0.14 & 0.15 & $\mathrm{NH}$ & 0.56 & 0.44 & 0.44 & 0.42 \\
$\mathrm{~F}_{2}$ & 0.02 & 0.02 & 0.00 & 0.01 & $\mathrm{NO}$ & -0.48 & -0.39 & -0.11 & 0.02 \\
$\mathrm{H}_{2}$ & 0.22 & 0.13 & 0.09 & 0.06 & $\mathrm{O}_{2}$ & 0.05 & 0.03 & 0.01 & 0.01 \\
$\mathrm{H}_{2} \mathrm{O}$ & 0.52 & 0.45 & 0.42 & 0.41 & $\mathrm{OF}$ & -0.02 & -0.02 & -0.01 & 0.00 \\
$\mathrm{H}_{2} \mathrm{O}_{2}$ & 0.52 & 0.44 & 0.39 & 0.36 & $\mathrm{OH}$ & 0.04 & 0.01 & 0.00 & -0.01 \\
\hline \hline
\end{tabular}

machinery ranging from EOM-CCSD (available using the xvcc, xecc and xncc modules), EOMCCSDT, and EOM-CCSDTQ (using the new xncc module) for high-accuracy calculations of core ionization and excitation energies. In order to eliminate spurious coupling between core ionized or excited states with high-lying valence excited states, Cederbaum and collaborators ${ }^{370}$ have pro- 
TABLE XI. Maximum absolute deviations (MAD) and standard deviations (SD) of CVS-EOM-CC ${ }^{373}$ and CVS- $\Delta \mathrm{CC}^{374}$ results from experimental values for chemical shifts of twenty-one 1s ionization energies of $\mathrm{C}, \mathrm{N}, \mathrm{O}, \mathrm{F}$ in fourteen molecules (in $\mathrm{eV}$ ).

\begin{tabular}{ccc}
\hline \hline & SD & MAD \\
\hline CVS-EOM-CCSD & 0.40 & 0.94 \\
CVS-EOM-CCSDT & 0.20 & 0.45 \\
CVS-EOM-CCSDTQ & 0.10 & 0.24 \\
\hline CVS- $\Delta$ HF & 0.70 & 1.67 \\
CVS- $\Delta$ CCSD & 0.19 & 0.53 \\
CVS- $\Delta$ CCSD(T) & 0.10 & 0.20 \\
\hline \hline
\end{tabular}

posed a generic idea of core-valence separation (CVS). The original formulation of CVS neglects coupling between core and valence orbitals in Hamiltonian integrals. An efficient implementation of this scheme has recently been reported by Vidal et al. for the EOM-CCSD method. ${ }^{371}$ We have adopted a variant of CVS suggested for EOM-CCSD by Coriani and Koch, ${ }^{372}$ in which CVS is only applied to the EOM vectors, i.e., only excitation operators containing targeted core orbitals are retained in the EOM vectors. EOM-CC methods using this variant of CVS (hereafter referred to as "CVS-EOM-CC" methods) have initially been implemented in CFOUR by using a projector that sets pure valence excitations in the EOM vector to zero in a regular EOM-CC calculation. As shown in Table VIII, benchmark studies have demonstrated the systematic convergence of CVS-EOM-CC methods and the high accuracy of computed core ionization energies when triples contributions are taken into account. 373

We have also recently explored the use of both perturbative and iterative approximations to full CVS-EOM-CCSDT, coupled with efficient techniques for implementing the core-valence separation for higher-order excitation amplitudes. ${ }^{375}$ Among the best-performing approximations was the CVS-EOM-CCSD* method, which is a straightforward modification of the original method of Stanton and Gauss. ${ }^{28}$ We have recently implemented these approximations in xncc (along with full CVS-EOM-CCSDT and CVS-EOM-CCSDTQ) using an algorithm that explicitly discards triple and quadruple excitation amplitudes with only valence occupied or inactive core indices. When only a constant number of core orbitals are active (in most calculations only one core orbital is active), this implementation leads to reduced scaling of the EOM-CC calculation. Importantly, the 
scaling is reduced to fully $M^{6}$ for CVS-EOM-CCSD*.

Although EOM-CC methods are capable of providing accurate results for core ionization energies, relatively slow convergence of computed results with respect to rank of excitation has been observed. This can be attributed to strong relaxation of the wavefunction due to the removal of core electron(s). The convergence is expected to be even slower for calculation of double core hole states. An alternative option for computing core ionization energies using $\Delta \mathrm{CC}$ methods ${ }^{374,376}$ has also been implemented in CFOUR and will be available in the next release. $\triangle \mathrm{CC}$ methods perform separate HF and CC calculations for the neutral molecule and the core-ionized state. Due to the local nature of core holes, the HF wavefunction of a core-ionized state can usually be obtained using maximum overlap method. ${ }^{377}$ The convergence problem of CC equations for core-ionized states due to coupling to valence continuum states can be handled using a generalization of the CVS scheme. ${ }^{374}$ Favorable accuracy has been obtained for CVS- $\Delta$ CC results of core ionization energies, with CVS- $\triangle \mathrm{CCSD}(\mathrm{T})$ providing results as accurate as CVS-EOM-CCSDTQ, as shown in Table VIII.

\section{Vibrational Perturbation Theory and Effective Hamiltonians}

CFOUR allows for the determination of harmonic vibrational frequencies for a wide range of quantum-chemical methods. When analytic Hessians are not available, the Hessian may be computed numerically by finite differences of gradients and/or single-point energies. Additionally, anharmonic vibrational frequencies and intensities may be obtained by finite differences (preferably of analytical Hessians). The xcubic module calculates anharmonic contributions based on secondorder vibrational perturbation theory (VPT2). ${ }^{380-384}$ While VPT2, when paired with a sufficient level of electron correlation and basis set completeness, can provide highly-accurate frequencies and intensities compared to gas-phase experiments, ${ }^{385-390}$ the presence of near-degeneracies in the harmonic frequencies can lead to a breakdown in the perturbation theory. Most commonly, VPT2 is affected by Fermi ${ }^{391}$ and Darling-Dennison ${ }^{392}$ resonances (although the latter is better described as a missing vibrational interaction rather than a PT breakdown). xcubic automatically attempts to detect cases of Fermi resonance and provides "deperturbed" frequencies and intensities, but a more accurate treatment requires the construction and diagonalization of an effective vibrational Hamiltonian as in contact transformation perturbation theory (Van Vleck perturbation theory). ${ }^{393,394}$ 
TABLE XII. Stretching levels of water, obtained at the CCSD(T) level of theory with the ANO2 basis set. Italicized level energies correspond to states of $b_{2}$ vibrational symmetry. The VPT2 values are ordered in terms of decreasing $v_{1}$ quantum numbers (i.e. the $3 v_{O H}$ levels are ordered 300, 201, 102 and 003), and the VPT2+K levels are ordered in terms of those with dominant eigenvector projections along the same zeroth-order levels.

\begin{tabular}{|c|c|c|c|c|c|c|c|c|}
\hline & \multicolumn{2}{|c|}{$v_{O H}$} & \multicolumn{2}{|c|}{$2 v_{O H}$} & \multicolumn{2}{|c|}{$3 v_{O H}$} & \multicolumn{2}{|c|}{$4 v_{O H}$} \\
\hline & VPT2 & JPT2+K & VPT2 & VPT2+K & VPT2 & VPT2+K & VPT2 & VPT2+K \\
\hline \multirow[t]{5}{*}{ Calc. } & 3659 & - & 7231 & 7201 & 10718 & 10591 & 14119 & 14215 \\
\hline & 3757 & - & 7249 & 7249 & 10656 & 10604 & 13977 & 13804 \\
\hline & - & - & 7415 & 7445 & 10742 & 10869 & 13982 & 13801 \\
\hline & - & - & - & - & 10976 & 11028 & 14136 & 14309 \\
\hline & - & - & - & - & - & - & 14439 & 14525 \\
\hline \multirow[t]{5}{*}{ Expt. $^{a}$} & 3657 & - & - & 7202 & - & 10600 & - & 13828 \\
\hline & 3756 & - & - & 7250 & - & 10613 & - & 13831 \\
\hline & - & - & - & 7445 & - & 10869 & - & 14221 \\
\hline & - & - & - & - & - & 11032 & - & 14319 \\
\hline & - & - & - & - & - & - & - & 14538 \\
\hline
\end{tabular}

${ }^{a}$ Refs. 378, 379

In order to treat these more difficult cases, the xguinea module is provided as a standalone program. xguinea reads the output of an anharmonic calculation, in particular the files rota, coriolis, dipole[xyz], quadratic, cubic, and quartic. The CFOUR job archive files (JOBARC and JAINDX) are used if present to determine symmetry and axis frame information. xguinea offers an interactive command-line input so that different options and structures of the effective Hamiltonian can be quickly explored. Alternatively, an input file can be fed to xguinea using shell redirection, e.g. xguinea < input. An example input file for treating multiple Fermi resonances in formaldehyde is given below (here $\omega_{5} \approx \omega_{2}+\omega_{6} \approx \omega_{3}+\omega_{6}$-the Darling-Dennison coupling between the latter two states is also included). The full xguinea manual is available on the CFOUR website (see Appendix 1). 
states

3

$\begin{array}{llllll}0 & 0 & 0 & 0 & 1 & 0\end{array}$

$\begin{array}{lllllll}0 & 1 & 0 & 0 & 0 & 1\end{array}$

$\begin{array}{lllllll}0 & 0 & 1 & 0 & 0 & 1\end{array}$

vibration

vpt2

diagonalize

calc

The construction of the effective Hamiltonian requires two steps: first, the diagonal elements are set equal to the deperturbed anharmonic frequencies. These differ from the standard VPT2 frequencies by removal of terms with a nearly-degenerate energy denominator. Second, the offdiagonal elements are determined by coupling formulas specific to the type of resonance (Fermi or Darling-Dennison) and the relationship between the two states. The Fermi coupling coefficients, also called the $F$ coefficients, are simply equal to scaled cubic force constants. The DarlingDennison, or $K$ coefficients, ${ }^{395}$ are much more complicated in form, and arise from the secondorder transformed Hamiltonian. The expressions for the effective Hamiltonian for the formaldehyde example above are given in Ref. 390,

$$
\begin{aligned}
\mathbf{H}_{\text {eff }} & =\left(\begin{array}{ccc}
5_{1} & 2{ }_{1} 6_{1} & 3_{1} 6_{1} \\
v_{5}^{*} & \frac{1}{\sqrt{8}} \phi_{256} & \frac{1}{\sqrt{8}} \phi_{356} \\
\frac{1}{\sqrt{8}} \phi_{256} & v_{2}+v_{6}+x_{26}^{*} & K \\
\frac{1}{\sqrt{8}} \phi_{356} & K & v_{3}+v_{6}+x_{36}^{*}
\end{array}\right) \\
K & =\frac{1}{4} \sum_{i=1}^{6} K_{i 2, i 3}+\frac{1}{2} K_{26,36}
\end{aligned}
$$

where an asterisk indicates deperturbation of the frequencies or anharmonicity coefficients $x_{i j}$, and the $K_{i j, k l}$ coefficients are tabulated in the literature. ${ }^{63,395,396}$

The treatment of Darling-Dennison resonances is especially important for accurately calculating the overtone and combination bands of molecules with multiple hydrogen stretching modes. For example, in water, the symmetric and antisymmetric $\mathrm{O}-\mathrm{H}$ stretching modes interact strongly for $2 v_{O H}$ and higher. Results from Ref. 63 for the $n v_{O H}, n=1,2,3,4$ levels of water computed 
with $\operatorname{CCSD}(\mathrm{T}) / \mathrm{ANO} 2^{397}$ are reproduced in Table XII. Overtone levels of $v_{3}$ are reproduced extremely well, as are combination and $v_{1}$ overtone levels for $v_{O H} \leq 3$. In the $4 v_{O H}$ polyad, additional interactions with bending mode overtones $n v_{2}$ begin to affect the symmetric stretching mode. Effective Hamiltonians for fixed polyad numbers are easily specified in xguinea, e.g.,

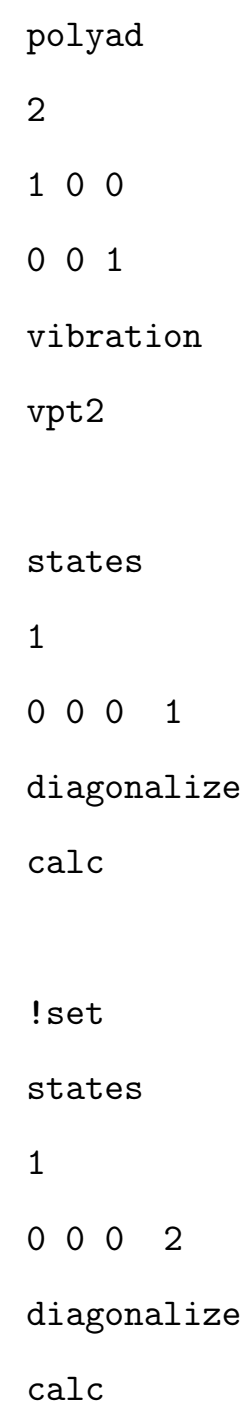

In addition to computing anharmonic frequencies, intensities, and vibrationally-averaged dipole moments at the VPT2 level, xguinea can also compute frequencies using fourth-order vibrational perturbation theory (VPT4). ${ }^{398}$ VPT4 calculations in xguinea additionally require the didq, quintic, and sextic files - the latter two are not calculated as part of a standard CFOUR anharmonic calculation, but they may be manually computed either by finite differences of fourth-order 
force fields or by fitting to a local potential energy surface (the "off-diagonal" quartic constants $\phi_{i j k l}$ with $i \neq j \neq k \neq l$ are also required). In a future version, we hope to extend xguinea to rotational and ro-vibrational spectroscopies and the calculation of higher-order vibration-rotation interaction and centrifugal distortion constants.

\section{FUTURE DIRECTIONS}

The discussion so far has focused on the current status of CFOUR, and is limited to features provided via either the current public version or a version to be released shortly. However, there are many other long-term developments concerning CFOUR either initially underway, or in the planning stages, that will extend its capabilities in the future. While most of these ideas are still in the planning stages and not yet appropriate for discussion, a few representative examples are provided here. Specifically, we will briefly discuss ongoing work on the use of Cholesky decomposition in order to facilitate computations on large molecules, and on the development of methods for treating atoms and molecules in the exotic but astrophysically relevant environment of finite and strong magnetic fields.

\section{A. Cholesky Decomposition representation of the electron repulsion integrals}

While the main focus of CFOUR is, by design, on the high-level treatment of small- to medium-sized molecules, extending the applicability of rigorous, ab initio methods to larger systems is becoming more and more desirable. The asymptotically rate-determining step of such calculations is the solution to amplitude equations, however, calculations on medium-large molecules with reasonable, but not too large basis sets, can often become overwhelming due to the cost of handling the two-electron repulsion integrals (ERIs). Operations such as the full or partial transformation of the ERIs from the AO to the MO basis may often become the limiting step in practice. This is due to two factors. First, it is usually safe to assume that the ERIs cannot fit in memory, which is usually true for ERIs in the AO basis and even more so for ERIs in the MO basis. This means that handling the integrals involves slow disk $\mathrm{I} / \mathrm{O}$, which can be a serious limiting factor. Second, integrals are computed (and stored) in an order that depends on the shell structure of the basis set, and accessed (or re-computed, for integral direct implementations ${ }^{399-401}$ ) in buffers. This makes writing subsequent code with optimal handling of memory accesses virtually impossible, 
as the order in which the integrals are available is system-dependent and in general not optimal for vectorization or use of highly optimized BLAS routines.

The ERI matrix is, however, not a full rank one. While there are in principle $\mathscr{O}\left(M^{4}\right)$ nonzero integrals, due to the localization of Gaussian basis functions, many of these will be small or negligible. ${ }^{399,400,402}$ This induces sparsity in the ERI matrix that can be exploited by introducing low rank approximations

$$
(\mu v \mid \rho \sigma) \approx \sum_{K L}^{n}(\mu v \mid K) S_{K L}(L \mid \mu v),
$$

where $n$ is the rank of the decomposition and is assumed to be much smaller than the full rank $N=M(M+1) / 2$, where $M$ is the number of basis functions. Popular choices are the so-called resolution of the identity (RI) ${ }^{403-407}$ (or density fitting) approximation and the Cholesky Decomposition $(\mathrm{CD})^{408-415}$ technique. In RI, an auxiliary basis set is introduced in order to approximate four-center integrals with products of three-center ones, according to Eq. 30. CD, on the other hand, is in principle the exact decomposition of the ERIs matrix in the product of a (full rank) lower triangular matrix times its transpose, i.e.,

$$
(\mu v \mid \rho \sigma)=\sum_{K=1}^{N} L_{\mu \nu}^{K} L_{\rho \sigma}^{K}
$$

However, the decomposition in Eq. 31 can be truncated at $n \ll N$ in a way that allows for both compression, to the point that the resulting Cholesky vectors can often be kept in memory, and a rigorous a priori control of the approximation error. The latter feature is particularly attractive, as the accuracy of a CD-based calculation can be precisely controlled, which is not the case for the RI approximation. On the other hand, RI computations can be performed using the same machinery used to compute the ERIs themselves, with little modifications, and many auxiliary basis sets are available in the literature, ${ }^{406,416-419}$ while CD needs an ad-hoc implementation and to compute the decomposition itself. The same applies for integral derivatives. ${ }^{420-422}$ We believe that this price is worth paying to retain full control on the precision of the calculation. For this reason, CD of the ERIs has been implemented in CFOUR.

The long term goal of this development is to offer all the main features of CFOUR in conjunction with a CD representation of the ERIs. CD allows for large computational savings in operations on the integral, as it reduces the scaling of AO to MO transformations from $M^{5}$ to $M^{4}$. However, it does not change the scaling of the correlated treatment, with the exception of scaled-opposite-spin second-order many body perturbation theory (SOS-MP2). ${ }^{423,424}$ Nevertheless, it can make a large 
difference as a formulation based on the $\mathrm{CD}$ of integrals is intrinsically well suited for writing all the operations involving the ERIs as matrix-matrix multiplications, that can be performed with very efficient level 3 BLAS routines. Furthermore, as each Cholesky vector $L^{K}$ contributes to the final quantity independently of the others, it is possible to parallelize CD-based calculations by distributing the Cholesky vectors.

At the moment, we are just starting to explore the use of CD in CFOUR. ${ }^{425}$ A particularly promising development is the coupling of $\mathrm{CD}$ with quadratically convergent solvers for both SCF and CASSCF. To show an example of the potential benefits of such a technique, we present here some preliminary results obtained with a serial, CD-based implementation of quadratically convergent SCF. This implementation is part of the experimental xdqcscf code described in section IV B.

As an example of the use of $\mathrm{CD}$ to extend the applicability of methods implemented in CFOUR, let us consider a medium-sized molecule such as caffeine $\left(\mathrm{C}_{8} \mathrm{H}_{10} \mathrm{~N}_{4} \mathrm{O}_{2}\right)$. Using Dunning's correlation consistent cc-pVTZ basis set, 560 basis functions are used, which is starting to be borderline for many post-HF applications. SCF optimization can however still be performed using the AO-based code in xdqcscf. The calculation requires, on a single core, about 2.5 hours, and is heavily dominated by disk I/O. The same calculation using CD ERIs can be performed in little more than 5 minutes, using a threshold for the $\mathrm{CD}$ of $10^{-4}$, which is a reasonable choice for most applications. All the timings were obtained on a single core of an Intel Xeon Gold 6140M processor. The main difference is that the CD ERI can easily be fitted in memory, avoiding thus slow I/O operations, and that the vast majority of the operations performed are done with highly efficient BLAS-3 routines. It is interesting to note that the same calculation, when performed forcing the use of an out-of-core algorithm and thus reading the Cholesky vectors from disk, requires slightly less than 10 minutes on the same machine. Therefore, even though the calculation is a factor of 2 slower than the same performed with the Cholesky vectors in core, it is still much faster than the traditional one. As a second example, we computed the SCF wavefunction for taxol $\left(\mathrm{C}_{47} \mathrm{H}_{51} \mathrm{NO}_{14}\right)$, a large molecule for which we employ again Dunning's cc-pVTZ basis set and the same settings for CD, for a total of 1947 basis functions. The SCF optimization can be performed in under 4.5 hours on the same cluster node used before. While these are very preliminary results and simple-minded applications, we believe that they offer a convincing argument in favor of using $\mathrm{CD}$ as a method to handle larger molecular systems.

We have also recently completed an implementation in xncc of a CD-based algorithm for the 
expensive particle-particle ladder contribution to MP3 and CCSD which avoids explicit storage of the $\langle a b \| c d\rangle$ integrals. We plan to extend this pilot implementation to additional terms in CCSD equations that deal with the $\langle a b|| c i\rangle$ integrals in order to further reduce storage and I/O bottlenecks.

\section{B. Reduced-Scaling Coupled Cluster Methods}

While the Cholesky decomposition approach (or RI/DF) can drastically reduce the memory and I/O requirements of both the SCF and correlated calculations, by itself it cannot reduce the scaling of post-Hartree-Fock methods except for SOS-MP2. In order to reduce the scaling of the same-spin (exchange) part of the MP2 energy as well, Hohenstein, et al. introduced a further factorization of the ERIs termed the tensor hypercontraction (THC) decomposition, ${ }^{426}$

$$
(\mu v \mid \rho \sigma) \approx \sum_{R S} X_{\mu}^{R} X_{v}^{R} V_{R S} X_{\rho}^{S} X_{\sigma}^{S}
$$

This factorization, combined with a Laplace quadrature representation of the orbital energy denominators reduces the scaling of full MP2 to $M^{4}$ and SOS-MP2 to $M^{3}$. Parrish et al. refined the THC method by assuming that the factor matrices $\mathbf{X}$ take the form of a real-space collocation of the orbitals over a set of grid points: $X_{\mu}^{R}=\phi_{\mu}\left(x_{R}\right) .{ }^{427}$ This reduces the problem of finding the interaction matrix $\mathbf{V}$ to a linear least squares problem with closed-form solution,

$$
\begin{aligned}
V_{R S} & =\sum_{R^{\prime} S^{\prime}} \sum_{\mu v \rho \sigma}\left(S^{-1}\right)_{R R^{\prime}} X_{\mu}^{R^{\prime}} X_{v}^{R^{\prime}}(\mu v \mid \rho \sigma) X_{\rho}^{S^{\prime}} X_{\sigma}^{S^{\prime}}\left(S^{-1}\right)_{S S^{\prime}} \\
S_{R S} & =\sum_{\mu \nu} X_{\mu}^{R} X_{v}^{R} X_{\mu}^{S} X_{v}^{S}
\end{aligned}
$$

This procedure scales as $M^{5}$ for exact ERIs but reduces to $M^{4}$ when paired with an additional $\mathrm{CD} / \mathrm{DF} / \mathrm{RI}$ approximation.

We have recently used this LS-THC factorization to implement reduced-scaling MP2 and MP3 methods (both scale as $M^{4}$ ). In particular, we have found that using a Cholesky decomposition of the real-space metric matrix $\mathbf{S}$ allows for defining "pruned" grids specific to particular classes of transformed MO integrals, e.g. $(a i \mid b j)$ vs. $(a b \mid c d) .{ }^{428}$ The accuracy of the LS-THC-DF-MP2 energy and size of the pruned grids were found to be similar or superior to hand-optimized 429 or other automatically-generated ${ }^{430,431}$ grids. We are now turning to the THC factorization of the double excitation amplitudes ${ }^{432}$ and the efficient implementation of a reduced-scaling THC-CCSD method. 


\section{Atoms and molecules in finite magnetic fields}

Strong magnetic fields lead, due to the interplay between Coulomb- and Lorentz forces, to a fascinating and complex electronic structure. ${ }^{433}$ For example, the lowest triplet state of the hydrogen molecule $\left({ }^{3} \Sigma_{u}^{+}\right)$becomes bound and even assumes the role of the ground state of the molecule at a sufficiently strong magnetic field by the so-called perpendicular paramagnetic bonding mechanism even though the formal bond order is zero. ${ }^{434}$ Such strong field strengths are of astrophysical relevance as they can be found on magnetic White Dwarf stars (WDs). Spectra from WDs are, however, very difficult to interpret since the magnetic field strength as well as the composition of the atmosphere are a priori unknown. As the magnetic field changes the electronic spectra completely, accurate quantum-chemical predictions are crucial prerequisites to interpretation. For such predictions, perturbation theory is inadequate because the field is by no means only a small perturbation to the system and finite-field methods have to be used instead. The predictions face the challenge that due to the structure of the Hamiltonian for a molecule in a magnetic field, the wave function becomes (in general) complex and therefore the implementation needs to allow for complex wavefunction parameters, integrals, etc. It is hence the goal to develop high-accuracy methods for the investigation of atoms and molecules in strong magnetic fields. Finite-field fullCI implementations exist and have led to the discovery of strongly magnetized WDs with helium atmospheres ${ }^{435}$ and the above-mentioned bonding mechanism. ${ }^{434}$ However, since finite-field fullCI only allows to study systems with very few electrons, alternative high-accuracy finite-field methods with lower computational scaling, such as finite-field methods based on coupled-cluster and equation-of-motion coupled-cluster theory are desirable. ${ }^{436-438}$ In order to use these methods within CFOUR, a new integral code using gauge-including atomic orbitals (GIAOs) based on the McMurchie Davidson scheme ${ }^{439,440}$ together with an SCF driver is being written and will be interfaced with the QCUMBRE program. ${ }^{441}$ The latter is written in C++ and designed in an object-oriented manner. A hierarchical data-type structure ensures that changes can be made on a low level without having to modify existing top-level code. A key feature of QCUMBRE is a black-box contraction routine that allows one to code in a manner that resembles the equations on paper while efficient complex BLAS algorithms like ZGEMM3M are being used internally to carry out matrix multiplications. 


\section{DATA AVAILABLITY STATEMENT}

Data available on request from the authors

\section{ACKNOWLEDGMENTS}

Those that have contributed to CFOUR extend well beyond the author list of this paper. In particular, JG, PGS and JFS would like to acknowledge R.J. Bartlett at the University of Florida, in whose research group the three were educated, allowed to flourish as postdoctorals, and to develop the bonds that ultimately led to their career-long collaboration. The many others that have contributed to CFOUR have made important developments that have paid benefits to all of us, and the complete list of authors can be found on the CFOUR website http://www.cfour.de.

CFOUR development in Gainesville is supported by the U.S. National Science Foundation, currently through Grant CHE-1664325. In Mainz, the work on CFOUR has been supported by the Deutsche Forschungsgemeinschaft, the Fonds der Chemischen Industrie, and the Alexander von Humboldt foundation. The CFOUR development in Budapest has been supported by the National Research, Innovation and Development Fund (NKFIA) of Hungary, Grant No. 124293. In Dallas, the CFOUR development was supported by a generous start-up grant from SMU and in Baltimore, the work on CFOUR has been supported by Department of Energy Early Career Research Program under contract number DE-SC0020317.

\section{Appendix A: Appendixes}

\section{Website and Online Documentation}

Already in 2005, at the time of the ACES II Mainz-Austin-Budapest (MAB) version, a wikibased website was implemented to replace the old latex based manual in order to increase the upto-dateness and to facilitate documentation of old and new features of the program package. With the renaming to CFOUR the current wiki-based website www.cfour.de was introduced, which provides detailed information how to obtain, install, and use the CFOUR program package, which features are available, as well as many illustrative examples together with a bibliography, which provides references for methods, basis sets, and the underlying implementations in CFOUR. 


\section{Mailing list}

Besides the aforementioned online manual (see Appendix 1), there is a mailing list available (cfour@lists.uni-mainz.de) to which any CFOUR user may subscribe. This mailing list, which is hosted at the University of Mainz, is meant as a forum for the exchange of experiences between users of the CFOUR program system. Users may join at any time via the website https://lists.unimainz.de/sympa/subscribe/cfour. Please note that in order to prevent spam, subscription requests are monitored and require that subscribers are accepted manually. After having subscribed, one can post questions and comments via email to the email address cfour@lists.uni-mainz.de. A searchable message archive of previous postings to the CFOUR mailing list, which goes back to about 2009, is available at https://lists.uni-mainz.de/sympa/arc/cfour.

\section{Licensing and Mode of Distribution}

For non-commercial purposes there is no charge to obtain CFOUR for academic users (individuals, universities, research institutes). The CFOUR license agreement, which is available from the aforementioned website, has to be signed and sent via regular mail or fax to the indicated address.

After reception of the properly signed unmodified CFOUR license agreement, instructions will be provided for downloading CFOUR from a GitLab server hosted by the University of Florida. This portal offers a user interface similar to other popular git-based portals such as GitHub and Bitbucket. From there, users can easily download any released CFOUR version. Bug fixes that fall between versions are distributed through this system as well, and users can either download a new version or receive updates through git version control.

\section{REFERENCES}

${ }^{1}$ J. F. Stanton, J. Gauss, L. Cheng, M. E. Harding, D. A. Matthews, and P. G. Szalay, "CFOUR, Coupled-Cluster techniques for Computational Chemistry, a quantum-chemical program package," With contributions from A.A. Auer, R.J. Bartlett, U. Benedikt, C. Berger, D.E. Bernholdt, Y.J. Bomble, O. Christiansen, F. Engel, R. Faber, M. Heckert, O. Heun, M. Hilgenberg, C. Huber, T.-C. Jagau, D. Jonsson, J. Jusélius, T. Kirsch, K. Klein, W.J. Lauderdale, F. Lipparini, T. Metzroth, L.A. Mück, D.P. O’Neill, D.R. Price, E. Prochnow, C. Puzzarini, K. Ruud, F. 
Schiffmann, W. Schwalbach, C. Simmons, S. Stopkowicz, A. Tajti, J. Vázquez, F. Wang, J.D. Watts and the integral packages MOLECULE (J. Almlöf and P.R. Taylor), PROPS (P.R. Taylor), ABACUS (T. Helgaker, H.J. Aa. Jensen, P. Jørgensen, and J. Olsen), and ECP routines by A. V. Mitin and C. van Wüllen. For the current version, see http://www.cfour.de.

${ }^{2}$ G. D. Purvis III and R. J. Bartlett, “ACES, a program to perform MBPT and CC calculations," Quantum Theory Project, University of Florida, Gainesville, FL, 1977-1989.

${ }^{3}$ R. J. Bartlett, "Many-body perturbation theory and coupled cluster theory for electron correlation in molecules," Ann. Rev. Phys. Chem. 32, 359-401 (1981).

${ }^{4}$ I. Shavitt and R. J. Bartlett, Many-Body Methods in Chemistry and Physics: MBPT and Coupled-Cluster Theory (Cambridge University Press, 2009).

${ }^{5}$ J. Gauss, J. F. Stanton, and R. J. Bartlett, "Coupled-cluster open-shell analytic gradients implementation of the direct product decomposition approach in energy gradient calculations," J. Chem. Phys. 95, 2623-2638 (1991).

${ }^{6}$ J. F. Stanton, J. Gauss, J. D. Watts, and R. J. Bartlett, “A direct product decomposition approach for symmetry exploitation in many-body methods. I. Energy calculation,” J. Chem. Phys. 94, 4334-4345 (1991).

${ }^{7}$ J. Almlöf, “The MOLECULE integral program,” Technical reports, Institute of Physics, University of Stockholm, Sweden, 1972 and 1974.

${ }^{8}$ P. R. Taylor, "VPROPS, a program for the evaluation of one-electron property integrals over Gaussians.".

${ }^{9}$ J. W. Moskowitz and L. C. Snyder, "POLYATOM: A general computer program for ab initio calculations," in Methods of Electronic Structure Theory. Modern Theoretical Chemistry, Vol 3, edited by H. F. Schaefer III (Springer, Boston, 1977) pp. 387-411.

${ }^{10}$ T. Helgaker, H. J. Aa. Jensen, P. Jørgensen, J. Olsen, and P. R. Taylor, “ABACUS, a Gaussian integral and integral derivative program.".

${ }^{11}$ J. D. Watts, J. F. Stanton, J. Gauss, and R. J. Bartlett, "A coupled-cluster study of the ground state of $C_{3}^{+}$," J. Chem. Phys. 94, 4320-4327 (1991).

${ }^{12}$ J. F. Stanton, J. Gauss, and R. J. Bartlett, "Potential nonrigidity of the $\mathrm{NO}_{3}$ radical," J. Chem. Phys. 94, 4084-4087 (1991).

${ }^{13}$ J. F. Stanton, J. Gauss, R. J. Bartlett, T. Helgaker, P. Jørgensen, H. J. Aa. Jensen, and P. R. Taylor, “Interconversion of diborane(4) isomers," J. Chem. Phys. 97, 1211-1216 (1992).

${ }^{14}$ J. F. Stanton, J. Gauss, J. D. Watts, W. J. Lauderdale, and R. J. Bartlett, "The ACES II program 
system," Int. J. Quantum Chem. 44, 879-894 (1992).

${ }^{15}$ V. Lotrich, N. Flocke, M. Ponton, A. Yau, A. Perera, E. Deumens, and R. J. Bartlett, "Parallel implementation of electronic structure energy, gradient and Hessian calculations," J. Chem. Phys. 128, 194104 (2008).

${ }^{16}$ J. Gauss, J. F. Stanton, M. E. Harding, and P. G. Szalay, "Coupled cluster techniques for computational chemistry," (2008), invited lecture at the 8th WATOC meeting in Sydney, Australia.

${ }^{17}$ J. Gauss, "Calculation of NMR chemical shifts at second-order many-body perturbation theory using gauge-including atomic orbitals," Chem. Phys. Lett. 191, 614-620 (1992).

${ }^{18} \mathrm{~J}$. Gauss, "Effects of electron correlation in the calculation of nuclear magnetic resonance chemical shifts," J. Chem. Phys. 99, 3629-3643 (1993).

${ }^{19}$ J. Gauss, "GIAO-MBPT(3) and GIAO-SDQ-MBPT(4) calculations of nuclear magnetic shielding constants," Chem. Phys. Lett. 229, 198-203 (1994).

${ }^{20}$ J. Gauss and J. F. Stanton, “Gauge-invariant calculation of nuclear magnetic shielding constants at the coupled-cluster singles and doubles level,” J. Chem. Phys. 102, 251-253 (1995).

${ }^{21}$ J. Gauss and J. F. Stanton, "Coupled-cluster calculations of nuclear magnetic resonance chemical shifts," J. Chem. Phys. 103, 3561-3578 (1995).

${ }^{22}$ J. Gauss and J. F. Stanton, "Perturbative treatment of triple excitations in coupled-cluster calculations of nuclear magnetic shielding constants," J. Chem. Phys. 104, 2574-2583 (1996).

${ }^{23}$ J. Gauss, "Analytic second derivatives for the full coupled-cluster singles, doubles, and triples model: Nuclear magnetic shielding constants for $\mathrm{BH}, \mathrm{HF}, \mathrm{CO}, \mathrm{N}_{2}, \mathrm{~N}_{2} \mathrm{O}$, and $\mathrm{O}_{3}$," J. Chem. Phys. 116, 4473-4776 (2002).

${ }^{24}$ J. F. Stanton and R. J. Bartlett, "The equation of motion coupled-cluster method. A systematic biorthogonal approach to molecular excitation energies, transition probabilities, and excited state properties," J. Chem. Phys. 98, 7029-7039 (1993).

${ }^{25}$ J. F. Stanton, "Many-body methods for excited state potential energy surfaces. I. General theory of energy gradients for the equation-of-motion coupled-cluster method," J. Chem. Phys. 99, 8840-8847 (1993).

${ }^{26}$ J. F. Stanton and J. Gauss, "Analytic energy gradients for the equation-of-motion coupledcluster method: Implementation and application to the HCN/HNC system," J. Chem. Phys. 100, 4695-4698 (1994).

${ }^{27}$ J. F. Stanton and J. Gauss, "Analytic energy derivatives for ionized states described by the equation-of-motion coupled cluster method," J. Chem. Phys. 101, 8938-8944 (1994). 
${ }^{28}$ J. F. Stanton and J. Gauss, "A simple correction to final state energies of doublet radicals described by equation-of-motion coupled cluster theory in the singles and doubles approximation," Theor. Chem. Acc. 93, 303-313 (1996).

${ }^{29}$ D. A. Matthews and J. F. Stanton, “A new approach to approximate equation-of-motion coupled cluster with triple excitations," J. Chem. Phys. 145, 124102 (2016).

${ }^{30}$ J. Gauss and J. F. Stanton, “Analytic $\operatorname{CCSD}(\mathrm{T})$ second derivatives," Chem. Phys. Lett. 276, 70-77 (1997).

${ }^{31}$ P. G. Szalay, J. Gauss, and J. F. Stanton, "Analytic UHF-CCSD(T) second derivatives: Implementation and application to the calculation of the vibration-rotation interaction constants of NCO and NCS," Theor. Chem. Acc. 100, 5-11 (1998).

${ }^{32}$ J. Gauss and J. F. Stanton, "Analytic first and second derivatives for the CCSDT-n ( $\left.n=1-3\right)$ models: a first step towards the efficient calculation of CCSDT properties," Phys. Chem. Chem. Phys. 2, 2047-2059 (2000).

${ }^{33}$ J. F. Stanton and J. Gauss, "Analytic second derivatives in high-order many-body perturbation and coupled-cluster theories: Computational considerations and applications," Int. Rev. Phys. Chem. 19, 61-96 (2000).

${ }^{34}$ J. F. Stanton, C. L. Lopreore, and J. Gauss, "The equilibrium structure and fundamental vibrational frequencies of dioxirane," J. Chem. Phys. 108, 7190-7196 (1998).

${ }^{35}$ C. Puzzarini, J. F. Stanton, and J. Gauss, "Quantum-chemical calculation of spectroscopic parameters for rotational spectroscopy,” Int. Rev. Phys. Chem. 29, 273-367 (2010).

${ }^{36}$ P. G. Szalay and J. Gauss, "Spin-restricted open-shell coupled-cluster theory," J. Chem. Phys. 107, 9028-9038 (1997).

${ }^{37}$ M. Heckert, O. Heun, P. G. Szalay, and J. Gauss, "Towards a spin-adapted coupled-cluster theory for high-spin open-shell states," J. Chem. Phys. 124, 124105 (2006).

${ }^{38}$ J. Gauss, K. Ruud, and T. Helgaker, "Perturbation-dependent atomic orbitals for the calculation of spin-rotation constants and rotational g tensors," J. Chem. Phys. 105, 2804-2812 (1996).

${ }^{39}$ J. Gauss and D. Sundholm, "Coupled-cluster calculations of spin-rotation constants," Mol. Phys. 91, 449-458 (1997).

${ }^{40}$ J. Gauss, M. Kállay, and F. Neese, "Calculation of electronic $g$-tensors using coupled cluster theory," J. Phys. Chem. A 113, 111541 (2009).

${ }^{41}$ G. Tarczay, P. G. Szalay, and J. Gauss, "First-principles calculation of electron spin-rotation tensors," J. Phys. Chem. A 114, 9246-9252 (2010). 
${ }^{42}$ M. Kállay, P. R. Nagy, Z. Rolik, D. Mester, G. Samu, J. Csontos, J. Csóka, B. P. Szabó, L. Gyevi-Nagy, I. Ladjánszki, L. Szegedy, B. Ladóczki, K. Petrov, M. Farkas, P. D. Mezei, and B. Hégely, “MRCC, a quantum chemical program.” See also Z. Rolik, L. Szegedy, I. Ladjánszki, B. Ladóczki, and M. Kállay, J. Chem. Phys. 139, 094105 (2013), as well as: www.mrcc.hu.

${ }^{43}$ M. Kállay, P. R. Nagy, D. Mester, Z. Rolik, G. Samu, J. Csontos, J. Csóka, P. B. Szabó, L. Gyevi-Nagy, B. Hégely, I. Ladjánszki, L. Szegedy, B. Ladóczki, K. Petrov, M. Farkas, P. D. Mezei, and Á. Ganyecz, "The MRCC program system: Accurate quantum chemistry from water to proteins," J. Chem. Phys. 152, accepted for publication, doi: 10.1063/1.5142048 (2020).

${ }^{44}$ M. Kállay and P. R. Surján, "Higher excitations in coupled-cluster theory," J. Chem. Phys. 115, 2945-2954 (2001).

${ }^{45}$ M. Kállay, J. Gauss, and P. G. Szalay, "Analytic first derivatives for general coupled-cluster and configuration interaction models,” J. Chem. Phys. 119, 2991-3004 (2003).

${ }^{46}$ M. Kállay and J. Gauss, "Analytic second derivatives for general coupled-cluster and configuration-interaction models," J. Chem. Phys. 120, 6841-6848 (2004).

${ }^{47}$ M. Kállay and J. Gauss, "Calculation of excited-state properties using general coupled-cluster and configuration-interaction models," J. Chem. Phys. 121, 9257-9269 (2004).

${ }^{48}$ J. Gauss, A. Tajti, M. Kállay, J. F. Stanton, and P. G. Szalay, "Analytic calculation of the diagonal Born-Oppenheimer correction within configuration-interaction and coupled-cluster theory," J. Chem. Phys. 125, 144111 (2006).

${ }^{49}$ A. Tajti, P. G. Szalay, and J. Gauss, "Perturbative treatment of the electron-correlation contribution to the diagonal Born-Oppenheimer correction,” J. Chem. Phys. 127, 014102 (2007).

${ }^{50}$ T. Ichino, J. Gauss, and J. F. Stanton, "Quasidiabatic states described by coupled-cluster theory," J. Chem. Phys. 130, 174105 (2011).

${ }^{51}$ A. Tajti and P. G. Szalay, "Analytic evaluation of the nonadiabatic coupling vector between excited states using equation-of-motion coupled-cluster theory," J. Chem. Phys. 131, 124104 (2009).

${ }^{52}$ S. Stopkowicz and J. Gauss, "Relativistic corrections to electrical first-order properties using direct perturbation theory," J. Chem. Phys. 129, 164119 (2008).

${ }^{53}$ S. Stopkowicz and J. Gauss, "Direct perturbation theory in terms of energy derivatives: Fourthorder relativistic corrections at the Hartree-Fock level,’ J. Chem. Phys. 129, 164119 (2011).

${ }^{54}$ S. Stopkowicz and J. Gauss, "Fourth-order relativistic corrections to electrical properties using 
Direct Perturbation Theory,” J. Chem. Phys. 134, 204106 (2011).

${ }^{55} \mathrm{~L}$. Cheng and J. Gauss, "Analytical evaluation of first-order electrical properties based on the spin-free Dirac-Coulomb Hamiltonian,” J. Chem. Phys. 134, 244112 (2011).

${ }^{56} \mathrm{~L}$. Cheng and J. Gauss, "Analytic energy gradients for the spin-free exact two-component theory using an exact block diagonalization for the one-electron Dirac Hamiltonian," J. Chem. Phys. 135, 084114 (2011).

${ }^{57}$ L. Cheng and J. Gauss, "Analytic second derivatives for the spin-free exact two-component theory,” J. Chem. Phys. 135, 2441045 (2011).

${ }^{58}$ L. Cheng, S. Stopkowicz, and J. Gauss, "Analytic energy derivatives in relativistic quantum chemistry," Int. J. Quantum Chem. 114, 1108-1127 (2014).

${ }^{59}$ J. Liu and L. Cheng, "An atomic mean-field spin-orbit approach within exact two-component theory for a non-perturbative treatment of spin-orbit coupling," J. Chem. Phys. 148, 144108 (2018).

${ }^{60} \mathrm{~A}$. Asthana, J. Liu, and L. Cheng, "Exact two-component equation-of-motion coupled-cluster singles and doubles method using atomic mean-field spin-orbit integrals," J. Chem. Phys. 150, 074102 (2019).

${ }^{61}$ A. Köhn, M. Hanauer, L. A. Mück, T.-C. Jagau, and J. Gauss, "State-specific multireference coupled-cluster theory," WIREs Comp. Mol. Sci. 3, 176-197 (2013).

${ }^{62}$ D. A. Matthews and J. F. Stanton, "Non-orthogonal spin-adaptation of coupled cluster methods: A new implementation of methods including quadruple excitations," J. Chem. Phys. 142, 064108 (2015).

${ }^{63}$ D. A. Matthews, J. Vazquéz, and J. F. Stanton, "Calculated stretching overtone levels and Darling-Dennison resonances in water: A triumph of simple theoretical approaches," Mol. Phys. 105, 19-22 (2007).

${ }^{64}$ D. A. Matthews and J. F. Stanton, "Quantitative analysis of Fermi resonances by harmonic derivatives of perturbation theory corrections," Mol. Phys. 107, 213-222 (2009).

${ }^{65}$ M. E. Harding, T. Metzroth, J. Gauss, and A. A. Auer, "Parallel calculation of CCSD and CCSD(T) analytic first and second derivatives," J. Chem. Theory Comput. 4, 64-74 (2008).

${ }^{66}$ E. Prochnow, M. E. Harding, and J. Gauss, "Parallel calculation of CCSDT and MkMRCCSDT energies," J. Chem. Theory Comput. 6, 2339-2347 (2010).

${ }^{67}$ I. M. Mills, "Vibration-rotation structure in asymmetric- and symmetric-top molecules," in Molecular Spectroscopy: Modern Research, edited by K. N. Rao and C. W. Mathews (Aca- 
demic Press, New York, 1972) pp. 115-140.

${ }^{68}$ M. Heckert, M. Kállay, D. P. Tew, W. Klopper, and J. Gauss, "Basis-set extrapolation techniques for the accurate calculation of molecular equilibrium geometries using coupled-cluster theory,” J. Chem. Phys. 125, 044108 (2006).

${ }^{69}$ J. Jusélius, D. Sundholm, and J. Gauss, "Calculation of current densities using gauge-including atomic orbitals," J. Chem. Phys. 121, 3952-3963 (2004).

${ }^{70}$ M. Barbatti, G. Granucci, M. Persico, M. Ruckenbauer, M. Vazdar, M. Eckert-Maksic, and H. Lischka, "The on-the-fly surface-hopping program system NEWTON-X: Application to ab initio simulation of the nonadiabatic photodynamics of benchmark systems," J. Photochem. Photobiol. A 190, 228-240 (2007).

${ }^{71}$ M. Barbatti, M. Ruckenbauer, F. Plasser, J. Pittner, G. Granucci, M. Persico, and H. Lischka, "Newton-X: a surface-hopping program for nonadiabatic molecular dynamics," WIREs Comp. Mol. Sci. 4, 26-33 (2014).

${ }^{72}$ M. Barbatti, G. Granucci, M. Ruckenbauer, F. Plasser, R. Crespo-Otero, J. Pittner, M. Persico, and H. Lischka, "NEWTON-X: A package for Newtonian dynamics close to the crossing Seam (v. 2.2). Available via the internet at www.newtonx.org," (2018).

${ }^{73}$ M. Hanauer and A. Köhn, "Pilot applications of internally contracted multireference coupled cluster theory, and how to choose the cluster operator properly," J. Chem. Phys. 134, 204111 (2011).

${ }^{74}$ F. Lipparini, T. Kirsch, A. Köhn, and J. Gauss, "Internally contracted multireference coupled cluster calculations with a spin-free Dirac-Coulomb Hamiltonian: Application to the monoxides of titanium, zirconium, and hafnium," J. Chem. Theory Comput. 13, 3171-3184 (2017).

${ }^{75}$ A. I. Krylov, "Equation-of-motion coupled-cluster methods for open-shell and electronically excited species: The hitchhiker's guide to Fock space," Ann. Rev. Phys. Chem. 59, 433-462 (2008).

${ }^{76}$ P. G. Szalay and R. J. Bartlett, “Analytic energy gradients for the 2-determinant coupled-cluster method with application to singlet excited-states of butadiene and ozone," J. Chem. Phys. 101, 4936-4944 (1994).

${ }^{77}$ F. Lipparini and J. Gauss, "Cost-effective treatment of scalar relativistic effects for multireference systems: A CASSCF implementation based on the spin-free Dirac-Coulomb Hamiltonian,” J. Chem. Theory Comput. 12, 4284-4295 (2016).

${ }^{78}$ D. Cremer, "Møller-Plesset perturbation theory: from small molecule methods to methods for 
thousands of atoms," WIREs Comp. Mol. Sci. 1, 509-530 (2000).

${ }^{79}$ T. D. Crawford and H. F. Schaefer III, “An introduction to coupled cluster theory for computational chemists," Rev. Comp. Chem. 14, 33-136 (2000).

${ }^{80}$ R. J. Bartlett and M. Musiał, "Coupled-cluster theory in quantum chemistry," Rev. Mod. Phys. 79, 291-352 (2007).

${ }^{81}$ F. Coester, "Bound states of a many-particle system," Nucl. Phys. 1, 421-424 (1958).

${ }^{82}$ F. Coester and H. Kümmel, "Short-range correlations in nuclear wave functions," Nucl. Phys. 17, 477-485 (1960).

${ }^{83}$ J. Č́ižek, "On the correlation problem in atomic and molecular systems. calculation of wavefunction components in Ursell-type expansion using quantum-field theoretical methods," J. Chem. Phys. 45, 4256-4266 (1966).

${ }^{84} \mathrm{~J}$. Č́žzek, "On the use of the cluster expansion and the technique of diagrams in calculations of correlation effects in atoms and molecules," Adv. Chem. Phys. 14, 35-89 (1966).

${ }^{85}$ K. Raghavachari, G. W. Trucks, J. A. Pople, and M. Head-Gordon, "A fifth-order perturbation comparison of electron correlation theories," Chem. Phys. Lett. 157, 479-483 (1989).

${ }^{86}$ J. A. Pople, J. S. Binkley, and R. Seeger, "Theoretical models incorporating electron correlation,” Int. J. Quantum Chem. Symp. 10, 1-19 (1976).

${ }^{87}$ R. J. Bartlett, "Coupled-cluster approach to molecular structure and spectra: A step toward predictive quantum chemistry,” J. Phys. Chem. 93, 1697-1708 (1989).

${ }^{88}$ P. R. Taylor, G. B. Bacskay, N. S. Hush, and A. Hurley, "The coupled-pair approximation in a basis of independent-pair natural orbitals," Chem. Phys. Lett. 41, 444-449 (1976).

${ }^{89}$ R. J. Bartlett and G. D. Purvis III, "Many-body perturbation theory, coupled-pair many-electron theory, and the importance of quadruple excitations for the correlation problem,” Int. J. Quantum Chem. 14, 561-581 (1978).

${ }^{90}$ J. A. Pople, R. Krishnan, H. B. Schlegel, and J. S. Binkley, "Electron correlation theories and their application to the study of simple reaction potential surfaces," Int. J. Quantum Chem. 14, 545-560 (1978).

${ }^{91}$ G. D. Purvis III and R. J.Bartlett, "A full coupled-cluster singles and doubles model: The inclusion of disconnected triples," J. Chem. Phys. 76, 1910-1918 (1982).

${ }^{92}$ J. Noga and R. J. Bartlett, “The full CCSDT model for molecular electronic structure,” J. Chem. Phys. 86, 7041-7050 (1987).

${ }^{93}$ G. E. Scuseria and H. F. Schaefer III, "A new implementation of the full CCSDT model for 
molecular electronic structure," Chem. Phys. Lett. 152, 382-386 (1988).

${ }^{94}$ N. Oliphant and L. Adamowicz, "Coupled-cluster method truncated at quadruples," J. Chem. Phys. 95, 6645-6651 (1991).

${ }^{95}$ S. A. Kucharski and R. J. Bartlett, "Recursive intermediate factorization and complete computational linearization of the coupled-cluster single, double, triple, and quadruple excitation equations," Theor. Chem. Acc. 80, 387-405 (1991).

${ }^{96}$ G. E. Scuseria, A. C. Scheiner, T. J. Lee, J. E. Rice, and H. F. Schaefer III, "The closedshell coupled cluster single and double excitation (CCSD) model for the description of electron correlation. A comparison with configuration interaction (CISD) results," J. Chem. Phys. 86, 2881 (1987).

${ }^{97}$ J. D. Watts and R. J. Bartlett, "The coupled-cluster single, double, and triple excitation model for open-shell single reference functions," J. Chem. Phys. 93, 6104 (1990).

${ }^{98}$ Y. S. Lee, S. A. Kucharski, and R. J. Bartlett, “A coupled cluster approach with triple excitations," J. Chem. Phys. 81, 5906-5912 (1984).

${ }^{99}$ J. Noga, R. J. Bartlett, and M. Urban, “Towards a full CCSDT model for electron correlation. CCSDT-n models," Chem. Phys. Lett. 134, 126-132 (1987).

${ }^{100}$ H. Koch, O. Christiansen, P. Jørgensen, A. Sánchez de Merás, and T. Helgaker, "The CC3 model: An iterative coupled cluster approach including connected triples,' J. Chem. Phys. 106, 1808-1818 (1997).

${ }^{101}$ O. Christiansen, C. Hättig, and P. Jørgensen, "Response functions from fourier component variational perturbation theory applied to a time-averaged quasienergy,” Int. J. Quantum Chem. 68, 1-52 (1998).

${ }^{102} \mathrm{O}$. Christiansen, H. Koch, and P. Jørgensen, “The second-order approximate coupled cluster singles and doubles model CC2," Chem. Phys. Lett. 243, 409-418 (1995).

${ }^{103}$ J. A. Pople, M. Head-Gordon, and K. Raghavachari, "Quadratic configuration interaction. a general technique for determining electron correlation energies," J. Chem. Phys. 87, 5968 (1979).

${ }^{104}$ K. Raghavachari, "An augmented coupled cluster method and its application to the first-row homonuclear diatomics," J. Chem. Phys. 82, 4607-4610 (1985).

${ }^{105}$ R. J. Bartlett, J. D. Watts, S. A. Kucharski, and J. Noga, "Non-iterative fifth-order triple and quadruple excitation energy corrections in correlated methods," Chem. Phys. Lett. 165, 513$522(1990)$. 
${ }^{106}$ J. F. Stanton, “Why CCSD(T) works: A different perspective," Chem. Phys. Lett. 281, 130 (1997).

${ }^{107}$ J. J. Eriksen, K. Kristensen, T. Kjæegaard, P. Jørgensen, and J. Gauss, “A Lagrangian framework for deriving triples and quadruples corrections to the CCSD energy," J. Chem. Phys. 140, 064108 (2014).

${ }^{108}$ Y. J. Bomble, J. F. Stanton, M. Kállay, and J. Gauss, "Coupled-cluster methods including noniterative corrections for quadruple excitations," J. Chem. Phys. 123, 054101 (2005).

${ }^{109}$ M. Kállay and J. Gauss, "Approximate treatment of higher excitations in coupled-cluster theory," J. Chem. Phys. 123, 214105 (2005).

${ }^{110}$ The reader is doubtless also aware of another popular class of elaborated triples correction, specifically those based on the completely renormalized (CR) class of methods (see, e.g. Ref. ? ). Indeed the CR-CC $(2,3)$ method is among the most popular methods other than $\operatorname{CCSD}(\mathrm{T})$ that is used for this purpose, but CFOUR does not yet have an implementation of this method.

${ }^{111}$ T. D. Crawford and J. F. Stanton, "Investigation of an asymmetric triple-excitation correction for coupled-cluster energies," Int. J. Quantum Chem. 70, 601-611 (1998).

${ }^{112}$ S. A. Kucharski and R. J. Bartlett, "Noniterative energy corrections through fifth-order to the coupled cluster singles and doubles method," J. Chem. Phys. 108, 5243-5254 (1998).

${ }^{113}$ M. Rittby and R. J. Bartlett, “An open-shell spin-restricted coupled cluster method: application to ionization potentials in nitrogen,” J. Phys. Chem. 92, 3033-3036 (1988).

${ }^{114}$ J. D. Watts, J. Gauss, and R. J. Bartlett, "Coupled-cluster methods with noniterative triple excitations for restricted open-shell Hartree-Fock and other general single determinant reference functions. Energies and analytical gradients," J. Chem. Phys. 98, 8718-8733 (1993).

${ }^{115}$ M. Kállay and J. Gauss, “Approximate treatment of higher excitations in coupled-cluster theory. II. Extension to general single-determinant reference functions and improved approaches for the canonical Hartree-Fock case,” J. Chem. Phys. 129, 144101 (2008).

${ }^{116}$ P. J. Knowles, J. S. Andrews, R. D. Amos, N. C. Handy, and J. A. Pople, "Restricted Møller-Plesset theory for open-shell molecules,” Chem. Phys. Lett. 186, 130-136 (1991).

${ }^{117}$ R. A. Chiles and C. E. Dykstra, "An electron pair operator approach to coupled cluster wave functions. Application to $\mathrm{He}_{2}, \mathrm{Be}_{2}$, and $\mathrm{Mg}_{2}$ and comparison with CEPA methods," J. Chem. Phys. 74, 4544-4556 (1981).

${ }^{118}$ J. F. Stanton, J. Gauss, and R. J. Bartlett, "On the choice of orbitals for symmetry breaking problems with application to $\mathrm{NO}_{3}$," J. Chem. Phys. 97, 5554-5559 (1992). 
${ }^{119}$ G. E. Scuseria and H. F. Schaefer III, "The optimization of molecular orbitals for coupled cluster wavefunctions," Chem. Phys. Lett. 142, 354 (1987).

${ }^{120} \mathrm{C}$. Møller and M. S. Plesset, "Note on an approximation treatment for many-electron systems," Phys. Rev. 46, 618-622 (1934).

${ }^{121}$ R. J. Bartlett and D. M. Silver, "Pair-correlation energies in sodium hydride with many-body perturbation theory,” Phys. Rev. A 10, 1927-1931 (1976).

${ }^{122}$ R. Krishnan, M. J. Frisch, and J. A. Pople, "Contribution of triple substitutions to the electron correlation energy in fourth order perturbation theory," J. Chem. Phys. 72, 4244 (1980).

${ }^{123}$ S. A. Kucharski, J. Noga, and R. J. Bartlett, "Fifth-order many-body perturbation theory for molecular correlation energies," J. Chem. Phys. 90, 7282-7290 (1989).

${ }^{124}$ Z. He and D. Cremer, "Sixth-order many-body perturbation theory. I. Basic theory and derivation of the energy formula," Int. J. Quantum Chem. 59, 15-29 (1996).

${ }^{125}$ Z. He and D. Cremer, "Sixth-order many-body perturbation theory. II. Implementation and application,” Int. J. Quantum Chem. 59, 31-55 (1996).

${ }^{126}$ J. Olsen, O. Christiansen, H. Koch, and P. Jørgensen, "Surprising cases of divergent behavior in Møller-Plesset perturbation theory,” J. Chem. Phys. 105, 5082-5090 (1996).

${ }^{127}$ J. Olsen and P. Jørgensen, "Divergence in Møller-Plesset theory: A simple explanation based on a two-state model," J. Chem. Phys. 112, 9736-9748 (2000).

${ }^{128}$ P. J. Knowles, K. Somasundram, N. C. Handy, and K. Hirao, "The calculation of higher-order energies in the many-body perturbation theory series," Chem. Phys. Lett. 113, 8-12 (1985).

${ }^{129}$ N. C. Handy, P. J. Knowles, and K. Somasundram, "On the convergence of the Møller-Plesset perturbation series,” Theor. Chem. Acc. 68, 87-100 (1985).

${ }^{130}$ I. Hubač and P. Č́rsky, "Correlation energy of open-shell systems. application of the manybody Rayleigh-Schrödinger perturbation theory in the restricted Roothaan-Hartree-Fock formalism,” Phys. Rev. A 22, 2392 (1980).

${ }^{131}$ C. Murray and E. R. Davidson, "Perturbation theory for open shell systems," Chem. Phys. Lett. 187, 451-454 (1991).

${ }^{132}$ R. D. Amos, J. S. Andrews, N. C. Handy, and P. J. Knowles, "Open-shell Møller-Plesset perturbation theory," Chem. Phys. Lett. 185, 256-264 (1991).

${ }^{133}$ W. J. Lauderdale, J. F. Stanton, J. Gauss, J. D. Watts, and R. J. Bartlett, "Many-body perturbation theory with a restricted open-shell Hartree-Fock reference," Chem. Phys. Lett. 187, 21 (1991). 
${ }^{134}$ W. J. Lauderdale, J. F. Stanton, J. Gauss, J. D. Watts, and R. J. Bartlett, "Restricted openshell Hartree-Fock-based many-body perturbation theory: Theory and application of energy and gradient calculations," J. Chem. Phys. 97, 6606 (1992).

${ }^{135}$ J. J. Eriksen, D. A. Matthews, P. Jørgensen, and J. Gauss, "Communication: The performance of non-iterative coupled cluster quadruples models," J. Chem. Phys. 143, 041101 (2015).

${ }^{136}$ T. Helgaker, "Gradient theory," in The Encyclopedia of Computational Chemistry, edited by P. von R. Schleyer, N. L. Allinger, T. Clark, J. Gasteiger, P. A. Kollmann, H. F. Schaefer III, and P. R. Schreiner (Wiley, Chichester, 1998) pp. 1157-1169.

${ }^{137}$ P. Pulay, “Analytical derivatives, forces, force constants, molecular geometries, and related response properties in electronic structure theory,” WIREs Comp. Mol. Sci. 4, 169-181 (2014).

${ }^{138}$ J. Gauss, J. F. Stanton, and R. J. Bartlett, "Analytic evaluation of energy gradients at the coupled-cluster singles and doubles level using quasi-restricted Hartree-Fock open-shell reference functions," J. Chem. Phys. 95, 2639-2645 (1991).

${ }^{139}$ J. Gauss, W. J. Lauderdale, J. F. Stanton, J. D. Watts, and R. J. Bartlett, "Analytic energy gradients for open-shell coupled-cluster singles and doubles (CCSD) calculations using restricted open-shell Hartree-Fock (ROHF) reference functions," Chem. Phys. Lett. 182, 207 215 (1991).

${ }^{140}$ J. D. Watts, J. Gauss, and R. J. Bartlett, "Open-shell analytical energy gradients for triple excitation many-body, coupled-cluster methods: MBPT(4), CCSD+T(CCSD), CCSD(T), and QCISD(T),” J. Chem. Phys. 200, 1-7 (1992).

${ }^{141}$ J. Gauss and J. F. Stanton, "Analytic gradients for the coupled-cluster singles, doubles, and triples (CCSDT) model," J. Chem. Phys. 116, 1773-1782 (2002).

${ }^{142}$ R. Fletcher, Practical Methods of Optimization (Wiley, New York, 1987).

${ }^{143}$ C. J. Cerjan and W. H. Miller, “On finding transition states," J. Chem. Phys. 75, 2800-2806 (1981).

${ }^{144}$ A. C. Scheiner, G. E. Scuseria, J. E. Rice, T. J. Lee, and H. F. Schaefer III, “Analytic evaluation of energy gradients for the single and double excitation coupled cluster (CCSD) wave function: Theory and application,” J. Chem. Phys. 87, 5361-5373 (1987).

${ }^{145}$ E. A. Salter, G. W. Trucks, and R. J. Bartlett, "Analytic energy derivatives in many-body methods. I. First derivatives,” J. Chem. Phys. 90, 1752-1766 (1989).

${ }^{146}$ A. Dalgarno and A. L. Stewart, "A perturbation calculation of properties of the helium isoelectronic sequence,” Proc. Roy. Soc. (London) Ser. A 247, 245-259 (1958). 
${ }^{147}$ L. Adamowicz and R. J. Bartlett, "Analytical gradients for the coupled-cluster method," Int. J. Quantum Chem. Symp. 26, 245-254 (1984).

${ }^{148}$ T. Helgaker, "Simple derivation of the potential energy gradient for an arbitrary electronic wave function,” Int. J. Quantum Chem. 21, 939-940 (1982).

${ }^{149}$ P. Jørgensen and T. Helgaker, "Møller-Plesset energy derivatives," J. Chem. Phys. 89, 15601570 (1988).

${ }^{150}$ T. Helgaker and P. Jørgensen, “Analytical calculation of geometrical derivatives in molecular electronic structure theory,” Adv. Quant. Chem. 19, 188-245 (1988).

${ }^{151}$ J. Arponen, "Variational principles and linked-cluster $\exp (\mathrm{S})$ expansions for static and dynamic many-body problems," Ann. Phys. 151, 311-382 (1983).

${ }^{152}$ J. Gerrat and I. M. Mills, "Force constants and dipole-moment derivatives of molecules from perturbed Hartree-Fock calculations. I,” J. Chem. Phys. 49, 1719 (1968).

${ }^{153}$ J. A. Pople, R. Krishnan, H. B. Schlegel, and J. S. Binkley, "Derivative studies in Hartree-Fock and Møller-Plesset theories,” Int. J. Quantum Chem. Symp. 13, 225-241 (1979).

${ }^{154}$ N. C. Handy and H. F. Schaefer III, "On the evaluation of analytic energy derivatives for correlated wave functions," J. Chem. Phys. 81, 5031 (1984).

${ }^{155}$ J. Rice and R. D. Amos, "On the efficient evaluation of analytic energy gradients," Chem. Phys. Lett. 122, 585-590 (1985).

${ }^{156}$ R. J. Bartlett, "Analytical evaluation of gradients in coupled-cluster and many-body perturbation theory," in Geometrical Derivatives of Energy Surfaces and Molecular Properties, edited by P. Jørgensen and J. Simons (Reidel Publishing, Dordrecht, 1986) pp. 35-61.

${ }^{157}$ E. A. Salter and R. J. Bartlett, "Analytic energy derivatives in many-body methods. II. Second derivatives," J. Chem. Phys. 90, 1767-1773 (1989).

${ }^{158}$ H. Koch, H. J. Aa.. Jensen, P. Jørgensen, T. Helgaker, G. E. Scuseria, and H. F. Schaefer III, "Coupled cluster energy derivatives. analytic Hessian for the closed-shell coupled cluster singles and doubles wave function: Theory and applications," J. Chem. Phys. 92, 4924-4940 (1990).

${ }^{159}$ J. F. Stanton and J. Gauss, "Analytic evaluation of second derivatives of the energy: Computational strategies for the CCSD and CCSD(T) approximations," in Recent Advances in CoupledCluster Methods, edited by R. J. Bartlett (World Scientific, Singapore, 1997) pp. 49-79.

${ }^{160} \mathrm{~W}$. Schneider and W. Thiel, "Anharmonic force fields from analytic second derivatives: Method and application to methyl bromide," Chem. Phys. Lett. 157, 367-373 (1989). 
${ }^{161}$ E. A. Salter, H. Sekino, and R. J. Bartlett, "Property evaluation and orbital relaxation in coupled cluster methods," J. Chem. Phys. 87, 502-509 (1987).

${ }^{162}$ D. Thouless, "Stability conditions and nuclear rotations in the hartree-fock theory," Nucl. Phys. 21, 225-232 (1960).

${ }^{163}$ O. Christiansen, J. Gauss, and J. F. Stanton, “The effect of triple excitations in coupled cluster calculations of frequency-dependent polarizabilities," Chem. Phys. Lett. 292, 437-446 (1998).

${ }^{164}$ R. Kobayashi, H. Koch, and P. Jørgensen, "Calculation of frequency-dependent polarizabilities using coupled-cluster response theory," Chem. Phys. Lett. 219, 30-35 (1984).

${ }^{165} \mathrm{M}$. Kállay and J. Gauss, "Calculation of frequency-dependent polarizabilities using general coupled-cluster models," J. Mol. Struct. (THEOCHEM) 768, 71-77 (2006).

${ }^{166} \mathrm{C}$. Hättig, O. Christiansen, and P. Jørgensen, “Frequency-dependent first hyperpolarizabilities using coupled cluster quadratic response theory," Chem. Phys. Lett. 269, 428-434 (1997).

${ }^{167}$ J. Gauss, O. Christiansen, and J. F. Stanton, "Triple excitation effects in coupled-cluster calculations of frequency-dependent hyperpolarizabilities," Chem. Phys. Lett. 296, 117-124 (1998).

${ }^{168}$ D. P. O’Neill, M. Kállay, and J. Gauss, "Calculation of frequency-dependent hyperpolarizabilities using general coupled-cluster models," J. Chem. Phys. 127, 134109 (2007).

${ }^{169}$ D. P. O’Neill, M. Kállay, and J. Gauss, “Analytic evaluation of Raman intensities in coupledcluster theory," Mol. Phys. 105, 2447-2453 (2007).

${ }^{170}$ S. Coriani, P. Jørgensen, O. Christiansen, and J. Gauss, "Triple excitation effects in coupled cluster calculations of Verdet constants," Chem. Phys. Lett. 330, 463-470 (2000).

${ }^{171} \mathrm{~F}$. London, "Théorie quantique des courants interatomiques dans les combinaisons aromatiques,” J. Phys. Radium 8, 397-409 (1937).

${ }^{172}$ H. F. Hameka, "On the nuclear magnetic shielding in the hydrogen molecule," Mol. Phys. 1, 203-215 (1958).

${ }^{173}$ R. Ditchfield, "Molecular orbital theory of magnetic shielding and magnetic susceptibility," J. Chem. Phys. 56, 5688-5691 (1972).

${ }^{174}$ K. Wolinski, J. F. Hinton, and P. Pulay, "Efficient implementation of the gauge-independent atomic orbital method for NMR chemical shift calculations," J. Am. Chem. Soc. 112, 82518260 (1990).

${ }^{175}$ T. Helgaker and P. Jørgensen, “An electronic Hamiltonian for origin independent calculations of magnetic properties," J. Chem. Phys. 95, 2595-2601 (1991).

${ }^{176}$ J. Gauss, K. Ruud, and M. Kállay, “Gauge-origin independent calculation of magnetizabilities 
and rotational $g$ tensors at the coupled-cluster level,’ J. Chem. Phys. 127, 074101 (2007).

${ }^{177}$ M. Kollwitz and J. Gauss, "A direct implementation of the GIAO-MBPT(2) method for calculating NMR chemical shifts. Application to the naphthalenium and anthracenium ions," Chem. Phys. Lett. 260, 639-646 (1996).

${ }^{178}$ M. Kollwitz, M. Häser, and J. Gauss, "Non-Abelian point group symmetry in direct secondorder many-body perturbation theory calculations of NMR chemical shifts," J. Chem. Phys. 137, 084107 (2012).

${ }^{179}$ S. Loibl and M. Schütz, "NMR shielding tensors for density fitted local second-order MøllerPlesset perturbation theory using gauge including atomic orbitals," J. Chem. Phys. 137, 084107 (2012).

${ }^{180}$ G. L. Stoychev, A. A. Auer, and F. Neese, "Efficient and accurate prediction of nuclear magnetic resonance shielding tensors with double-hybrid density functional theory," J. Chem. Theory Comput. 14, 4756-4771 (2018).

${ }^{181}$ W. H. Flygare, "Magnetic interactions in molecules and an analysis of molecular electronic charge distribution from magnetic parameters," Chem. Rev. 74, 653-687 (1974).

${ }^{182}$ A. A. Auer and J. Gauss, "Triple excitation effects in coupled-cluster calculations of indirect spin-spin coupling constants," J. Chem. Phys. 115, 1619-1623 (2001).

${ }^{183}$ R. Faber, S. P. A. Sauer, and J. Gauss, "Importance of triples contributions to NMR spin-spin coupling constants computed at the CC3 and CCSDT levels," J. Chem. Theory Comput. 13, 696-709 (2017).

${ }^{184}$ S. A. Perera, H. Sekino, and R. J. Bartlett, "Coupled-cluster calculations of indirect nuclear coupling constants: The importance of non-Fermi contact contributions," J. Chem. Phys. 101, 2186-2191 (1994).

${ }^{185}$ S. A. Perera, M. Nooijen, and R. J. Bartlett, "Electron correlation effects on the theoretical calculation of nuclear magnetic resonance spin-spin coupling constants," J. Chem. Phys. 104, 3290-3305 (1996).

${ }^{186}$ A. A. Auer, J. Gauss, and J. F. Stanton, "Quantitative prediction of gas-phase ${ }^{13} \mathrm{C}$ nuclear magnetic shielding constants," J. Chem. Phys. 118, 10407-10417 (2003).

${ }^{187}$ H. J. Monkhorst, "Calculation of properties with the coupled-cluster method,” Int. J. Quantum. Chem. Symp. 11, 421-432 (1977).

${ }^{188}$ K. Emrich, "An extension of the coupled cluster formalism to excited states (I)," Nucl. Phys. A 351, 379-396 (1981). 
${ }^{189}$ D. C. Comeau and R. J. Bartlett, "The equation-of-motion coupled-cluster method. Applications to open- and closed-shell reference states," Chem. Phys. Lett. 207, 414-423 (1993).

${ }^{190}$ S. Ghosh, D. Mukherjee, and S. Bhattacharyya, "Application of linear response theory in a coupled cluster framework for the calculation of ionization potentials,” Mol. Phys. 43, 173-179 (1981).

${ }^{191}$ H. Sekino and R. J. Bartlett, "A linear response, coupled-cluster theory for excitation energy," Int. J. Quantum Chem. Symp. 18, 255-265 (1984).

${ }^{192}$ H. Koch and P. Jørgensen, “Coupled cluster response functions,” J. Chem. Phys. 93, 3333-3344 (1990).

${ }^{193}$ H. Koch, H. J. Aa.. Jensen, P. Jørgensen, and T. Helgaker, "Excitation energies from the coupled cluster singles and doubles linear response function (CCSDLR). Applications to $\mathrm{Be}, \mathrm{CH}^{+}$, CO, and $\mathrm{H}_{2} \mathrm{O}$,” J. Chem. Phys. 93, 3345-3350 (1990).

${ }^{194}$ R. J. Rico and M. Head-Gordon, "Single-reference theories of molecular excited states with single and double substitutions," Chem. Phys. Lett. 213, 224-232 (1993).

${ }^{195}$ H. Nakatsuji and K. Hirao, "Cluster expansion of the wavefunction. Symmetry-adapted-cluster expansion, its variational determination, and extension of open-shell orbital theory," J. Chem. Phys. 68, 2053-2065 (1978).

${ }^{196}$ N. Nakatsuji, "Cluster expansion of the wavefunction. Excited states," Chem. Phys. Lett. 59, 362-364 (1978).

${ }^{197}$ H. Nakatsuji, "Cluster expansion of the wavefunction. Electron correlations in ground and excited states by SAC (symmetry-adapted-cluster) and SAC CI theories," Chem. Phys. Lett. 67, 329-333 (1979).

${ }^{198}$ H. Nakatsuji, "Cluster expansion of the wavefunction. Calculation of electron correlations in ground and excited states by SAC and SAC CI theories," Chem. Phys. Lett. 67, 334-342 (1979).

${ }^{199}$ D. Kánnár and P. G. Szalay, "Benchmarking coupled cluster methods on valence singlet excited states," J. Chem. Theory Comput. 10, 3757-3765 (2014).

${ }^{200}$ D. Kánnár, A. Tajti, and P. G. Szalay, “Accuracy of coupled cluster excitation energies in diffuse basis sets," J. Chem. Theory Comput. 13, 202-209 (2017).

${ }^{201}$ R. Izsak, "Single-reference coupled cluster methods for computing excitation energies in large molecules: The efficiency and accuracy of approximations," WIREs Comp. Mol. Sci. , e1445 (2019).

${ }^{202}$ K. Kowalski and P. Piecuch, "The active-space equation-of-motion coupled-cluster methods for 
excited electronic states: Full EOMCCSDt," J. Chem. Phys. 115, 643-651 (2001).

${ }^{203}$ S. A. Kucharski, M. Włoch, M. Musiał, and R. Rodney J. Bartlett, "Coupled-cluster theory for excited electronic states: The full equation-of-motion coupled-cluster single, double, and triple excitation method," J. Chem. Phys. 115, 8263-8266 (2001).

${ }^{204}$ Y. J. Bomble, K. W. Sattelmeyer, J. F. Stanton, and J. Gauss, “On the vertical excitation energy of cyclopentadiene,” J. Chem. Phys. 121, 5236-5240 (2004).

${ }^{205}$ S. Hirata, "Higher-order equation-of-motion coupled-cluster methods," J. Chem. Phys. 121, 51 (2004).

${ }^{206}$ J. H. Baraban, D. A. Matthews, and J. F. Stanton, "Communication: An accurate calculation of the $\mathrm{S}_{1} \mathrm{C}_{2} \mathrm{H}_{2}$ cis-trans isomerization barrier height," J. Chem. Phys. 144, 111102 (2016).

${ }^{207}$ O. Christiansen, H. Koch, and P. Jørgensen, "Response functions in the CC3 iterative triple excitation model," J. Chem. Phys. 103, 7429-7441 (1995).

${ }^{208}$ J. D. Watts and R. J. Bartlett, "The inclusion of connected triple excitations in the equation-ofmotion coupled-cluster method," J. Chem. Phys. 101, 3073-3078 (1994).

${ }^{209}$ J. D. Watts and R. J. Bartlett, "Economical triple excitation equation-of-motion coupled-cluster methods for excitation energies," Chem. Phys. Lett. 233, 81-87 (1995).

${ }^{210}$ O. Christiansen, H. Koch, and P. Jørgensen, "Perturbative triple excitation corrections to coupled cluster singles and doubles excitation energies," J. Chem. Phys. 105, 1451-1459 (1996).

${ }^{211}$ J. D. Watts and R. J. Bartlett, "Iterative and non-iterative triple excitation corrections in coupledcluster methods for excited electronic states: The EOM-CCSDT-3 and EOM-CCSD(T) methods," Chem. Phys. Lett. 258, 581-588 (1996).

${ }^{212}$ S. Hirata, M. Nooijen, I. Grabowski, and R. J. Bartlett, "Perturbative corrections to coupledcluster and equation-of-motion coupled-cluster energies: A determinantal analysis," J. Chem. Phys. 114, 3919-3928 (2001).

${ }^{213} \mathrm{~K}$. Kowalski and P. Piecuch, "New type of noniterative energy corrections for excited electronic states: Extension of the method of moments of coupled-cluster equations to the equation-ofmotion coupled-cluster formalism,” J. Chem. Phys. 115, 2966-2978 (2001).

${ }^{214}$ T. J. Watson, Jr., V. F. Lotrich, P. G. Szalay, A. Perera, and R. J. Bartlett, "Benchmarking for perturbative triple-excitations in EE-EOM-CC methods,” J. Phys. Chem. A 117, 2569-2579 (2013).

${ }^{215}$ K. Kowalski and P. Piecuch, "New coupled-cluster methods with singles, doubles, and noniterative triples for high accuracy calculations of excited electronic states,” J. Chem. Phys. 120, 
$1715-1738$ (2004).

${ }^{216}$ M. W. Włoch, M. D. Lodriguito, P. Piecuch, and J. R. Gour, "Two new classes of non-iterative coupled-cluster methods derived from the method of moments of coupled-cluster equations," Mol. Phys. 104, 2149-2172 (2006); “Erratum,” 104, 2991-2991 (2006).

${ }^{217}$ P. U. Manohar and A. I. Krylov, "A noniterative perturbative triples correction for the spinflipping and spin-conserving equation-of-motion coupled-cluster methods with single and double substitutions," J. Chem. Phys. 129, 194105 (2008).

${ }^{218}$ P. U. Manohar, J. F. Stanton, and A. I. Krylov, "Perturbative triples correction for the equationof-motion coupled-cluster wave functions with single and double substitutions for ionized states: Theory, implementation, and examples," J. Chem. Phys. 131, 114112 (2009).

${ }^{219}$ A. Tajti, J. F. Stanton, D. A. Matthews, and P. G. Szalay, “Accuracy of coupled cluster excited state potential energy surfaces,” J. Chem. Theory Comput. 14, 5859-5869 (2018).

${ }^{220}$ T.-C. Jagau, "Non-iterative triple excitations in equation-of-motion coupled-cluster theory for electron attachment with applications to bound and temporary anions," J. Chem. Phys. 148, 024104 (2018).

${ }^{221} \mathrm{~L}$. Cheng, “A study of non-iterative triples contributions in relativistic equation-of-motion coupled-cluster calculations using an exact two-component Hamiltonian with atomic meanfield spin-orbit integrals: Application to uranyl and other heavy-element compounds," J. Chem. Phys. 151, 104103 (2019).

${ }^{222}$ K. W. Sattelmeyer, H. F. Schaefer III, and J. F. Stanton, "Use of $2 \mathrm{~h}$ and $3 \mathrm{~h}-\mathrm{p}$-like coupledcluster Tamm-Danncoff approaches for the equilibrium properties of ozone," Chem. Phys. Lett. 378, 42-46 (2003).

${ }^{223} \mathrm{M}$. Nooijen and R. J. Bartlett, "Equation of motion coupled cluster method for electron attachment,” J. Chem. Phys. 102, 3629-3647 (1995).

${ }^{224}$ J. F. Stanton and J. Gauss, "A simple scheme for the direct calculation of ionization potentials with coupled-cluster theory that exploits established excitation energy methods," J. Chem. Phys. 111, 8785-8788 (1999).

${ }^{225}$ J. E. Del Bene, R. Ditchfield, and J. A. Pople, "Self-consistent molecular orbital methods. X. Molecular orbital studies of excited states with minimal and extended basis sets," J. Chem. Phys. 55, 2236-2241 (1971).

${ }^{226}$ I. Tamm, "Relativistic interaction of elementary particles,” J. Phys. (USSR) 9, 449-460 (1945).

${ }^{227}$ S. M. Dancoff, “Non-adiabatic meson theory of nuclear forces," Phys. Rev. 78, 382-385 (1950). 
${ }^{228}$ M. Head-Gordon, R. J. Rico, M. Oumi, and T. J. Lee, "A doubles correction to electronic excited states from configuration interaction in the space of single substitutions," Chem. Phys. Lett. 219, 21-29 (1994).

${ }^{229}$ J. F. Stanton and J. Gauss, "Perturbative treatment of the similarity transformed Hamiltonian in equation-of-motion coupled-cluster approximations,” J. Chem. Phys. 103, 1064-1076 (1995).

${ }^{230}$ H. Koch, R. Kobayashi, A. Sánchez de Merás, and P. Jørgensen, "Calculation of size-intensive transition moments from the coupled cluster singles and doubles linear response function," J. Chem. Phys. 100, 4393-4400 (1994).

${ }^{231}$ S. Coriani, F. Pawłowski, J. Olsen, and P. Jørgensen, "Molecular response properties in equation of motion coupled cluster theory: A time-dependent perspective,” J. Chem. Phys. 144, 024102 (2016).

${ }^{232}$ J. J. Eriksen, P. Jørgensen, J. Olsen, and J. Gauss, “Equation-of-motion coupled cluster perturbation theory revisited," J. Chem. Phys. 140, 174114 (2014).

${ }^{233}$ A. Tajti, P. G. Szalay, A. Császár, M. Kállay, J. Gauss, E. F. Valeev, B. A. Flowers, J. Vázquez, and J. F. Stanton, "HEAT: High accuracy extrapolated ab initio thermochemistry," J. Chem. Phys. 121, 11599 (20004).

${ }^{234}$ Y. J. Bomble, J. Vázquez, M. Kállay, C. Michauk, P. G. Szalay, A. G. Császár, J. Gauss, and J. F. Stanton, "High-accuracy extrapolated ab initio thermochemistry. II. Minor improvements to the protocol and a vital simplification,” J. Chem. Phys. 125, 064108 (2006).

${ }^{235}$ M. E. Harding, J. Vázquez, B. Ruscic, A. K. Wilson, J. Gauss, and J. F. Stanton, "Highaccuracy extrapolated ab initio thermochemistry. III. Additional improvements and overview," J. Chem. Phys. 128, 114111 (2008).

${ }^{236}$ J. M. L. Martin and G. de Oliveira, "Towards standard methods for benchmark quality ab initio thermochemistry — W1 and W2 theory," J. Chem. Phys. 111, 1843-1856 (1999).

${ }^{237}$ A. D. Boese, M. Oren, O. Atasoylu, J. Martin, M. Kállay, and J. Gauss, "W3 theory: Robust computational thermochemistry in the $\mathrm{kJ} / \mathrm{mol}$ accuracy range," J. Chem. Phys. 120, 4129-4141 (2004).

${ }^{238}$ A. Karton, E. Rabinovich, J. M. L. Martin, and B. Ruscic, "W4 theory for computational thermochemistry: In pursuit of confident sub-kJ/mol predictions,” J. Chem. Phys. 125, 144108 (2006).

${ }^{239}$ S. J. Klippenstein, L. B. Harding, and B. Ruscic, "Ab initio computations and active thermochemical tables hand in hand: Heats of formation of core combustion species," J. Phys. Chem. 
A 121, 6580-6602 (2017).

${ }^{240}$ D. A. Matthews, J. Gauss, and J. F. Stanton, "Revisitation of nonorthogonal spin adaptation in coupled cluster theory,” J. Chem. Theory Comput. 9, 2567-2572 (2013).

${ }^{241}$ D. A. Matthews and J. F. Stanton, "Chapter 10 - diagrams in coupled-cluster theory: Algebraic derivation of a new diagrammatic method for closed shells," in Mathematical Physics in Theoretical Chemistry, Developments in Physical \& Theoretical Chemistry, edited by S. Blinder and J. House (Elsevier, 2019) pp. 327-375.

${ }^{242}$ S. A. Kucharski and R. J. Bartlett, "The coupled-cluster single, double, triple, and quadruple excitation method," J. Chem. Phys. 97, 4282-4288 (1992).

${ }^{243}$ J. C. Saeh and J. F. Stanton, "Application of an equation-of-motion coupled cluster method including higher-order corrections to potential energy surfaces of radicals," J. Chem. Phys. 111, 8275-8285 (1999).

${ }^{244}$ D. A. Matthews and J. F. Stanton, "Accelerating the convergence of higher-order coupled cluster methods," J. Chem. Phys. 143, 204103 (2015).

${ }^{245}$ D. Matthews, "High-performance tensor contraction without transposition," SIAM J. Sci. Comput. 40, C1-C24 (2018).

${ }^{246}$ D. A. Matthews, “On extending and optimising the direct product decomposition,” Mol. Phys. 117, 1325-1333 (2019).

${ }^{247}$ H.-J. Werner, "Matrix-formulated direct multiconfiguration self-consistent field and multiconfiguration reference configuration-interaction methods," Adv. Chem. Phys. 69, 1-62 (2007).

${ }^{248}$ R. Shepard, "The multiconfiguration self-consistent field method," Adv. Chem. Phys. 69, 63200 (2007).

${ }^{249}$ B. Levy and G. Berthier, "Generalized Brillouin theorem for multiconfigurational SCF theories,” Int. J. Quantum Chem. 2, 307-319 (1968).

${ }^{250}$ J. Hinze, "MC-SCF. I. The multi-configuration self-consistent-field method,” J. Chem. Phys. 59, 6424-6432 (1973).

${ }^{251}$ B. O. Roos, P. R. Taylor, and P. E. M. Siegbahn, "A complete active space SCF method (CASSCF) using a density matrix formulated super-CI approach," Chem. Phys. 48, 157-173 (1980).

${ }^{252}$ R. H. A. Eade and M. A. Robb, "Direct minimization in MC SCF theory. The quasi-Newton method," Chem. Phys. Lett. 83, 362-368 (1981).

${ }^{253}$ U. Meier and V. Staemmler, "An efficient first-order CASSCF method based on the renormal- 
ized Fock-operator technique," Theor. Chem. Acc. 76, 95-111 (1989).

${ }^{254}$ M. Frisch, I. N. Ragazos, M. A. Robb, and H. B. Schlegel, "An evaluation of three direct MC-SCF procedures," Chem. Phys. Lett. 189, 524-528 (1992).

${ }^{255}$ P. E. M. Siegbahn, J. Almlöf, A. Heiberg, and B. O. Roos, "The complete active space SCF (CASSCF) method in a Newton-Raphson formulation with application to the HNO molecule," J. Chem. Phys. 74, 2384-2396 (1981).

${ }^{256}$ H.-J. Werner and W. Meyer, "A quadratically convergent MCSCF method for the simultaneous optimization of several states," J. Chem. Phys. 74, 5794-5801 (1981).

${ }^{257}$ P. Jørgensen, P. Swanstrøm, and D. L. Yeager, "Guaranteed convergence in ground state multiconfigurational self-consistent field calculations," J. Chem. Phys. 78, 347-356 (1983).

${ }^{258}$ H. J. Aa. Jensen and H. Ågren, "MC SCF optimization using the direct, restricted step, secondorder norm-extended optimization method," Chem. Phys. Lett. 110, 140-144 (1984).

${ }^{259}$ H. J. Aa. Jensen and P. Jørgensen, “A direct approach to second-order mcscf calculations using a norm extended optimization scheme," J. Chem. Phys. 80, 1204-1214 (1984).

${ }^{260} \mathrm{H}$. J. Aa. Jensen and H. Ågren, “A direct, restricted-step, second-order MC SCF program for large scale ab initio calculations," Chem. Phys. 104, 229-250 (1986).

${ }^{261}$ H.-J. Werner and P. J. Knowles, "A second order multiconfiguration SCF procedure with optimum convergence,” J. Chem. Phys. 82, 5053-5063 (1985).

${ }^{262}$ N. C. Handy, "Multi-root configuration interaction calculations," Chem. Phys. Lett. 74, 280283 (1980).

${ }^{263}$ P. J. Knowles and N. C. Handy, "A new determinant-based full configuration interaction method," Chem. Phys. Lett. 111, 315-321 (1984).

${ }^{264}$ J. Olsen, B. O. Roos, P. Jørgensen, and H. J. Aa. Jensen, “Determinant based configuration interaction algorithms for complete and restricted configuration interaction spaces," J. Chem. Phys. 89, 2185-2192 (1988).

${ }^{265}$ G. L. Bendazzoli and S. Evangelisti, "A vector and parallel full configuration interaction algorithm," J. Chem. Phys. 98, 3141-3150 (1993).

${ }^{266}$ T. H. Dunning, Jr., "Gaussian basis sets for use in correlated molecular calculations. I. The atoms boron through neon and hydrogen,” J. Chem. Phys. 90, 1007-1023 (1989).

${ }^{267} \mathrm{P}$. Pulay, "Convergence acceleration of iterative sequences. The case of scf iteration," Chem. Phys. Lett. 73, 393-398 (1980).

${ }^{268}$ K. S. Pitzer, "Relativistic effects on chemical properties," Acc. Chem. Res. 12, 271-276 (1979). 
${ }^{269}$ P. Pyykkö, "Relativistic effects in structural chemistry," Chem. Rev. 88, 563-594 (1988).

${ }^{270}$ S. A. Perera and R. J. Bartlett, "Relativistic effects at the correlated level. An application to interhalogens," Chem. Phys. Lett. 216, 606-612 (1993).

${ }^{271}$ R. D. Cowan and D. C. Griffin, "Approximate relativistic corrections to atomic radial wave functions,” J. Opt. Soc. Am. 66, 1010-1014 (1976).

${ }^{272} \mathrm{C}$. Michauk and J. Gauss, "Perturbative treatment of scalar-relativistic effects in coupled-cluster calculations of equilibrium geometries and harmonic vibrational frequencies using analytic second-derivative techniques," J. Chem. Phys. 127, 044106 (2007).

${ }^{273}$ A. Rutkowski, "Relativistic perturbation theory. I. A new perturbation approach to the Dirac equation,” J. Phys. B At. Mol. Phys. 19, 149-158 (1986).

${ }^{274}$ W. Kutzelnigg, E. Ottschofski, and R. Franke, "Relativistic Hartree-Fock by means of stationary direct perturbation theory. I. General theory,” J. Chem. Phys. 102, 1740-1751 (1995).

${ }^{275}$ W. Klopper, "Simple recipe for implementing computation of first-order relativistic corrections to electron correlation energies in framework of direct perturbation theory," J. Comput. Chem. 18, 20-27 (1997).

${ }^{276}$ W. Schwalbach, S. Stopkowicz, L. Cheng, and J. Gauss, "Direct perturbation theory in terms of energy derivatives: Scalar-relativistic treatment up to sixth order," J. Chem. Phys. 135, 194114 (2011).

${ }^{277}$ L. Visscher, T. J. Lee, and K. G. Dyall, "Formulation and implementation of a relativistic unrestricted coupled-cluster method including noniterative connected triples,” J. Chem. Phys. 105, 8769-8776 (1996).

${ }^{278}$ K. G. Dyall, "Interfacing relativistic and nonrelativistic methods. IV. One- and two-electron scalar approximations,” J. Chem. Phys. 115, 9136-9143 (2001).

${ }^{279}$ W. Liu and D. Peng, "Exact two-component Hamiltonians revisited," J. Chem. Phys. 131, 1-5 (2009).

${ }^{280}$ S. Stopkowicz and J. Gauss, “A one-electron variant of direct perturbation theory for the treatment of scalar-relativistic effects," Mol. Phys. 117, 1242-1251 (2019).

${ }^{281}$ W. Zou, M. Filatov, and D. Cremer, "Development and application of the analytical energy gradient for the normalized elimination of the small component method," J. Chem. Phys. 134, 244117 (2011).

${ }^{282}$ K. G. Dyall, "An exact separation of the spin-free and spin-dependent terms of the DiracCoulomb-Breit Hamiltonian,” J. Chem. Phys. 100, 2118-2127 (1994). 
${ }^{283}$ J. Sikkema, L. Visscher, T. Saue, and M. Iliaš, "The molecular mean-field approach for correlated relativistic calculations," J. Chem. Phys. 131, 124116 (2009).

${ }^{284}$ T. Kirsch, F. Engel, and J. Gauss, “Analytic evaluation of first-order properties within the meanfield variant of spin-free exact two-component theory," J. Chem. Phys. 150, 204115 (2019).

${ }^{285}$ K. Fægri, "Relativistic Gaussian basis sets for the elements K - Uuo," Theor. Chem. Acc. 105, 252-258 (2001).

${ }^{286}$ B. O. Roos, R. Lindh, P.-Å. Malmqvist, V. Veryazov, and P.-O. Widmark, "New relativistic ANO basis sets for transition metal atoms," J. Phys. Chem. A 109, 6575-6579 (2005).

${ }^{287}$ L. Cheng, S. Stopkowicz, and J. Gauss, "Spin-free Dirac-Coulomb calculations augmented with a perturbative treatment of spin-orbit effects at the Hartree-Fock level," J. Chem. Phys. 139, 214114 (2013).

${ }^{288} \mathrm{~L}$. Cheng and J. Gauss, "Perturbative treatment of spin-orbit coupling within spin-free exact two-component theory," J. Chem. Phys. 141, 164107 (2014).

${ }^{289}$ L. Cheng, F. Wang, J. Stanton, and J. Gauss, "Perturbative treatment of spin-orbit-coupling within spin-free exact two-component theory using equation-of-motion coupled-cluster methods," J. Chem. Phys. 148, 044108 (2018).

${ }^{290}$ B. A. Heß, C. M. Marian, U. Wahlgren, and O. Gropen, "A mean-field spin-orbit method applicable to correlated wavefunctions," Chem. Phys. Lett. 251, 365-371 (1996).

${ }^{291} \mathrm{~F}$. Neese, "Efficient and accurate approximations to the molecular spin-orbit coupling operator and their use in molecular $g$-tensor calculations," J. Chem. Phys. 122, 034107 (2005).

${ }^{292}$ K. Klein and J. Gauss, "Perturbative calculation of spin-orbit splittings using the equation-ofmotion ionization-potential coupled-cluster ansatz," J. Chem. Phys. 129, 194106 (2008).

${ }^{293}$ E. Epifanovsky, K. Klein, S. Stopkowicz, J. Gauss, and A. I. Krylov, "Spin-orbit couplings within the equation-of-motion coupled-cluster framework: Theory, implementation, and benchmark calculations," J. Chem. Phys. 143, 064102 (2015).

${ }^{294}$ F. Wang, J. Gauss, and C. van Wüllen, "Closed-shell coupled-cluster theory with spin-orbit coupling," J. Chem. Phys. 129, 064113 (2008).

${ }^{295} \mathrm{~F}$. Wang and J. Gauss, "Analytic energy gradients in closed-shell coupled-cluster theory with spin-orbit coupling,” J. Chem. Phys. , 174110.

${ }^{296} \mathrm{~F}$. Wang and J. Gauss, "Analytic second derivatives in closed-shell coupled-cluster theory with spin-orbit coupling,” J. Chem. Phys. 131, 164113 (2009).

${ }^{297}$ Z. Tu, F. Wang, and X. Li, "Equation-of-motion coupled-cluster method for ionized states with 
spin-orbit coupling," J. Chem. Phys. 136, 174102 (2012).

${ }^{298}$ D.-D. Yang, F. Wang, and J. Guo, "Equation of motion coupled cluster method for electron attached states with spin-orbit coupling," Chem. Phys. Lett. 531, 236-241 (2012).

${ }^{299}$ Z. Wang, Z. Tu, and F. Wang, "Equation-of-motion coupled-cluster theory for excitation energies of closed-shell systems with spin-orbit coupling," J. Chem. Theory Comput. 10, 55675576 (2014).

${ }^{300}$ K. G. Dyall, "Interfacing relativistic and nonrelativistic methods. I. Normalized elimination of the small component in the modified Dirac equation,” J. Chem. Phys. 106, 9618-9626 (1997).

${ }^{301}$ W. Kutzelnigg and W. Liu, "Quasirelativistic theory equivalent to fully relativistic theory," J. Chem. Phys. 123, 241102 (2005).

${ }^{302}$ M. Iliaš and T. Saue, "An infinite-order two-component relativistic Hamiltonian by a simple one-step transformation," J. Chem. Phys. 126, 64102 (2007).

${ }^{303}$ W. Liu, "Ideas of relativistic quantum chemistry," Mol. Phys. 108, 1679-1706 (2010).

${ }^{304}$ T. Saue, "Relativistic Hamiltonians for Chemistry: A Primer," ChemPhysChem 12, 3077-3094 (2011).

${ }^{305}$ D. Peng and M. Reiher, "Exact decoupling of the relativistic Fock operator," Theor. Chem. Acc. 131, 1-20 (2012).

${ }^{306}$ J. Liu, Y. Shen, A. Asthana, and L. Cheng, "Two-component relativistic coupled-cluster methods using mean-field spin-orbit integrals,” J. Chem. Phys. 148, 034106 (2018).

${ }^{307}$ N. Oliphant and L. Adamowicz, "Multireference coupled-cluster method using a singlereference formalism,” J. Chem. Phys. 94, 1229-1236 (1991).

${ }^{308}$ M. Kállay, P. G. Szalay, and P. R. Surján, “A general state-selective multireference coupledcluster algorithm," J. Chem. Phys. 117, 980-991 (2002).

${ }^{309}$ D. I. Lyakh, M. Musiał, V. F. Lotrich, and R. J. Bartlett, "Multireference nature of chemistry: The coupled-cluster view," Chem. Rev. 2012, 182-243 (2012).

${ }^{310}$ U. S. Mahapatra, B. Datta, and D. Mukherjee, “A state-specific multi-reference coupled cluster formalism with molecular applications," Mol. Phys. 94, 157-171 (1998).

${ }^{311}$ U. S. Mahapatra, B. Datta, and D. Mukherjee, “A size-consistent state-specific multireference coupled cluster theory: Formal developments and molecular applications," J. Chem. Phys. 110, 6171-6188 (1999).

${ }^{312}$ B. Jeziorski and H. Monkhorst, "Coupled-cluster method for multideterminantal reference states," Phys. Rev. A 24, 1668-1681 (1981). 
${ }^{313}$ F. A. Evangelista, W. D. Allen, and H. F. Schaefer III, "Coupling term derivation and general implementation of state-specific multireference coupled cluster theories," J. Chem. Phys. 127, 024102 (2007).

${ }^{314}$ F. A. Evangelista, A. C. Simmonett, W. D. Allen, H. F. Schaefer III, and J. Gauss, “Triple excitations in state-specific multireference coupled cluster theory: Application of Mk-MRCCSDT and Mk-MRCCSDT-n methods to model systems," 2008 128, 124104 (2008).

${ }^{315}$ F. A. Evangelista and J. Gauss, "Insights into the orbital invariance problem in state-specific multireference coupled cluster theory," J. Chem. Phys. 133, 044101 (2010).

${ }^{316}$ T.-C. Jagau and J. Gauss, "Linear-response theory for Mukherjee's multireference coupledcluster method: Static and dynamic polarizabilities,” J. Chem. Phys. 137, 044115 (2012).

${ }^{317}$ T.-C. Jagau and J. Gauss, "Linear-response theory for Mukherjee's multireference coupledcluster method: Excitation energies," J. Chem. Phys. 137, 044116 (2012).

${ }^{318}$ E. Prochnow, F. A. Evangelista, H. F. Schaefer III, W. D. Allen, and J. Gauss, "Analytic gradients for the state-specific multireference coupled cluster singles and doubles model," J. Chem. Phys. 131, 064109 (2009).

${ }^{319}$ S. Das, D. Mukherjee, and M. Kállay, "Full implementation and benchmark studies of Mukherjee's state-specific multireference coupled-cluster ansatz," J. Chem. Phys. 132, 074103 (2010).

${ }^{320}$ F. A. Evangelista, E. Prochnow, J. Gauss, and H. F. Schaefer III, "Perturbative triples corrections in state-specific multireference coupled cluster theory," J. Chem. Phys. 132, 074107 (2010).

${ }^{321}$ T.-C. Jagau and J. Gauss, "Ground and excited state geometries via Mukherjee's multireference coupled-cluster method," Chem. Phys. 401, 73-87 (2012).

${ }^{322}$ A. Banerjee and J. Simons, "The coupled-cluster method with a multiconfiguration reference state,” Int. J. Quantum Chem. 19, 207-216 (1981).

${ }^{323}$ F. A. Evangelista and J. Gauss, “An orbital-invariant internally contracted multireference coupled cluster approach," J. Chem. Phys. 134, 114102 (2011).

${ }^{324}$ M. Hanauer and A. Köhn, "Perturbative treatment of triple excitations in internally contracted multireference coupled cluster theory,” J. Chem. Phys. 136, 204107 (2012).

${ }^{325}$ F. A. Evangelista, M. Hanauer, A. Köhn, and J. Gauss, “A sequential transformation approach to the internally contracted multireference coupled cluster method," J. Chem. Phys. 136, 204108 (2012).

${ }^{326}$ M. Hanauer and A. Köhn, "Restoring full size extensivity in internally contracted multirefer- 
ence coupled cluster theory," J. Chem. Phys. 137, 131103 (2012).

${ }^{327}$ T.-C. Jagau, E. Prochnow, F. A. Evangelista, and J. Gauss, “Analytic gradients for Mukherjee's multireference coupled-cluster method using two-configurational self-consistent-field orbitals," J. Chem. Phys. 132, 144110 (2010).

${ }^{328}$ E. Prochnow and J. Gauss, unpublished (2011).

${ }^{329}$ L. A. Mück and J. Gauss, "Spin-orbit splittings in degenerate open-shell states via Mukherjee's multireference coupled-cluster theory: A measure for the coupling contribution,' J. Chem. Phys. 136, 111103 (2012).

${ }^{330}$ H. Köppel, W. Domcke, and L. S. Cederbaum, "Multimode molecular dynamics beyond the Born-Oppenheimer approximation,” Adv. Chem. Phys. 57, 59-246 (1984).

${ }^{331}$ L. S. Cederbaum, W. Domcke, J. Schirmer, and W. van Niessen, "Correlation effects in the ionization of molecules: Breakdown of the molecular orbital picture," Adv. Chem. Phys. 65, 115-159 (1986).

${ }^{332}$ M. Mayer, L. S. Cederbaum, and H. Köppel, "Ground state dynamics of $\mathrm{NO}_{3}$ : Multimode vibronic borrowing including thermal effects," J. Chem. Phys. 100, 899-911 (1994).

${ }^{333}$ K. Klein, E. Garand, T. Ichino, D. M. Neumark, J. Gauss, and J. F. Stanton, "Quantitative vibronic coupling calculations: The formyloxyl radical," Theor. Chem. Acc. 129, 527-543 (2011).

${ }^{334}$ C. S. Simmons, T. Ichino, and J. F. Stanton, "The $v_{3}$ fundamental in $\mathrm{NO}_{3}$ has been seen near $1060 \mathrm{~cm}^{-1}$, albeit some time ago," J. Phys. Chem. Lett. 3, 1946-1950 (2012).

${ }^{335} \mathrm{O}$. Christiansen, "First-order nonadiabatic coupling matrix elements using coupled cluster methods. I. Theory,” J. Chem. Phys. 110, 711-723 (1999).

${ }^{336}$ S. Faraji, S. Matsika, and A. I. Krylov, "Calculations of non-adiabatic couplings within equation-of-motion coupled-cluster framework: Theory, implementation, and validation against multi-reference methods,” J. Chem. Phys. 148, 044103 (2018).

${ }^{337}$ J. F. Stanton, "Coupled-cluster theory, pseudo-Jahn-Teller effects and conical intersections," J. Chem. Phys. 115, 10382-10393 (2001).

${ }^{338}$ A. Weaver, R. B. Metz, S. E. Bradforth, and D. M. Neumark, "Observation of the $\mathrm{A}^{2} \mathrm{~B}_{2}$ and $\mathrm{C}^{2} \mathrm{~A}_{2}$ states of $\mathrm{NO}_{2}$ by negative ion photoelectron spectroscopy of $\mathrm{NO}_{2}^{-}$, J. Chem. Phys. 90, 2070-2071 (1989).

${ }^{339}$ S. M. Rabidoux, V. Eijkhout, and J. F. Stanton, "A highly-efficient implementation of the Doktorov recurrence equations for Franck-Condon calculations," J. Chem. Theory Comput. 12, 
728-739 (2016).

${ }^{340} \mathrm{D}$. Feller, "The use of systematic sequences of wave functions for estimating the complete basis set, full configuration interaction limit in water,” J. Chem. Phys. 98, 7059-7071 (1993).

${ }^{341}$ T. Helgaker, W. Klopper, H. Koch, and J. Noga, "Basis-set convergence of correlated calculations on water," J. Chem. Phys. 106, 9639-9646 (1997).

${ }^{342}$ M. S. Schuurman, S. R. Muir, W. D. Allen, and H. F. Schaefer III, "Toward subchemical accuracy in computational thermochemistry: Focal point analysis of the heat of formation of NCO and [H,N,C,O] isomers," J. Chem. Phys. 120, 11586-11599 (2004).

${ }^{343}$ D. Feller, K. A. Peterson, and D. A. Dixon, “A survey of factors contributing to accurate theoretical predictions of atomization energies and molecular structures," J. Chem. Phys. 129, 204105 (2008).

${ }^{344}$ M. Heckert, M. Kállay, and J. Gauss, "Molecular equilibrium geometries based on coupledcluster calculations including quadruple excitations," Mol. Phys. 13, 2109-2115 (2005).

${ }^{345}$ D. E. Woon and T. H. Dunning, Jr., "Gaussian basis sets for use in correlated molecular calculations. V. Core-valence basis sets for boron through neon,” J. Chem. Phys. 103, 4572-4585 (1995).

${ }^{346}$ L. A. Mück, V. Lattanzi, S. Thorwirth, M. C. McCarthy, and J. Gauss, "Cyclic $\mathrm{SiS}_{2}$ : A new perspective on the Walsh rules," Angew. Chem. Int. Ed. 51, 3695-3698 (2012).

${ }^{347}$ W. S. Benedict, N. Gailar, and E. K. Plyler, "Rotation-vibration spectra of deuterated water vapor," J. Chem. Phys. 24, 1139-1165 (1956).

${ }^{348}$ M. Born and R. Oppenheimer, "Zur Quantentheorie der Molekeln," Ann. Phys. 84, 457-484 (1927).

${ }^{349}$ H. Köppel and W. Domcke, "Vibronic dynamics of polyatomic molecules," in The Encyclopedia of Computational Chemistry, edited by P. von R. Schleyer, N. L. Allinger, T. Clark, J. Gasteiger, P. A. Kollmann, H. F. Schaefer III, and P. R. Schreiner (Wiley, Chichester, 1998) p. 3166.

${ }^{350} \mathrm{M}$. Born and K. Huang, Dynamical theory of crystal lattices (Oxford University Press, Oxford, 1954).

${ }^{351}$ J. R. Reimers, L. K. McKemmish, R. H. McKenzie, and N. S. Hush, "Non-adiabatic effects in thermochemistry, spectroscopy and kinetics: The general importance of all three Born-Oppenheimer breakdown corrections," Phys. Chem. Chem. Phys. 17, 24641-24665 (2015). 
${ }^{352}$ N. C. Handy and A. M. Lee, "The adiabatic approximation," Chem. Phys. Lett. 252, 425-430 (1996).

${ }^{353}$ W. Kutzelnigg, "The adiabatic approximation I. The physical background of the Born-Handy ansatz,” Mol. Phys. 90, 909-916 (1997).

${ }^{354}$ A. Karton, P. R. Taylor, and J. M. L. Martin, "Basis set convergence of post-CCSD contributions to molecular atomization energies," J. Chem. Phys. 127, 064104 (2007).

${ }^{355}$ A. Karton, "A computational chemist's guide to accurate thermochemistry for organic molecules," WIREs Comput. Mol. Sci. 6, 292-310 (2016).

${ }^{356}$ Á. Ganyecz, M. Kállay, and J. Csontos, "Moderate-cost ab initio thermochemistry with chemical accuracy," J. Chem. Theory Comput. 13, 4193-4204 (2017).

${ }^{357}$ F. Holka, P. G. Szalay, J. Fremont, M. Rey, K. A. Peterson, and V. G. Tyuterev, “Accurate $\mathrm{ab}$ initio determination of the adiabatic potential energy function and the Born-Oppenheimer breakdown corrections for the electronic ground state of LiH isotopologues," J. Chem. Phys. 134, 094306 (2011).

${ }^{358}$ C. Puzzarini and J. Gauss, "Quantum-chemical determination of Born-Oppenheimer breakdown parameters for rotational constants: The open-shell species $\mathrm{CN}, \mathrm{CO}^{+}$, and $\mathrm{BO},{ }^{\prime} \mathrm{Mol}$. Phys. 111, 2204-2210 (2013).

${ }^{359}$ C. Stein, O. Weser, B. Schröder, and P. Botschwina, "High-level theoretical spectroscopic parameters for three ions of astrochemical interest," Mol. Phys. 113, 2169-2178 (2015).

${ }^{360}$ O. L. Polyansky, R. I. Ovsyannikov, A. A. Kyuberis, L. Lodi, J. Tennyson, A. Yachmenev, S. N. Yurchenko, and N. F. Zobov, "Calculation of rotation-vibration energy levels of the ammonia molecule based on an ab initio potential energy surface," J. Mol. Spectr. 327, 21-30 (2016).

${ }^{361}$ A. V. Nikitin, M. Rey, and V. G. Tyuterev, "First fully ab initio potential energy surface of methane with a spectroscopic accuracy," J. Chem. Phys. 145, 114309 (2016).

${ }^{362}$ M. Gronowski, P. Eluszkiewicz, and T. Custer, "Structure and spectroscopy of $\mathrm{C}_{2} \mathrm{HNO}$ isomers," J. Phys. Chem. A 121, 3263-3273 (2017).

${ }^{363}$ A. Owens, A. Yachmenev, J. Küpper, S. N. Yurchenko, and W. Thiel, "The rotation-vibration spectrum of methyl fluoride from first principles," Phys. Chem. Chem. Phys. 21, 3496-3505 (2019).

${ }^{364} \mathrm{~J}$. Koput, “Ab initio structure and vibration-rotation dynamics of germylene, $\mathrm{GeH}_{2}$," J. Comput. Chem. 40, 1911-1918 (2019).

${ }^{365}$ K. Siegbahn, C. Nordling, A. Fahlman, R. Nordberg, K. Hamrin, J. Hedman, G. Johansson, 
T. Bergmark, S.-E. Karlsson, I. Lindgren, and B. Lindberg, ESCA. Atomic, Molecular and Solid State Structure Studied by Means of Electron Spectroscopy (Almqvist-Wiksells, Uppsala, 1967).

${ }^{366}$ K. Siegbahn, "Electron spectroscopy for atoms, molecules, and condensed matter," Science 217, 111-121 (1982), http://www.sciencemag.org/content/217/4555/111.full.pdf.

${ }^{367}$ L. Young, K. Ueda, M. Guehr, P. H. Bucksbaum, M. Simon, S. Mukamel, N. Rohringer, K. C. Prince, C. Masciovecchio, M. Meyer, A. Rudenko, D. Rolles, C. Bostedt, M. Fuchs, D. A. Reis, R. Santra, H. Kapteyn, M. Murnane, H. Ibrahim, F. Legare, M. Vrakking, M. Isinger, D. Kroon, M. Gisselbrecht, A. L'Huillier, H. J. Worner, and S. R. Leone, "Roadmap of ultrafast X-ray atomic and molecular physics," J. Phys. B-Atomic Mol. Opt. Phys. 51, 032003 (2018).

${ }^{368}$ P. M. Kraus, M. Zürch, S. K. Cushing, D. M. Neumark, and S. R. Leone, "The ultrafast X-ray spectroscopic revolution in chemical dynamics,” Nat. Rev. Chem. 2, 82-94 (2018).

${ }^{369}$ P. Norman and A. Dreuw, "Simulating X-ray spectroscopies and calculating core-excited states of molecules," Chem. Rev. 118, 7208-7248 (2018).

${ }^{370}$ L. S. Cederbaum, W. Domcke, and J. Schirmer, "Many-body theory of core holes," Phys. Rev. A At., Mol., Opt. Phys. 22, 206-222 (1980).

${ }^{371}$ M. L. Vidal, X. Feng, E. Epifanovsky, A. I. Krylov, and S. Coriani, "New and Efficient Equation-of-Motion Coupled-Cluster Framework for Core-Excited and Core-Ionized States," J. Chem. Theory Comput. 15, 3117-3133 (2019).

${ }^{372}$ S. Coriani and H. Koch, "Communication: X-ray absorption spectra and core-ionization potentials within a core-valence separated coupled cluster framework," J. Chem. Phys. 143, 181103 (2015).

${ }^{373}$ J. Liu, D. Matthews, S. Coriani, and L. Cheng, "Benchmark calculations of K-edge ionization energies for first-row elements using scalar-relativistic core-valence-separated equationof-motion coupled-cluster methods," J. Chem. Theory Comput. 15, 1642-1651 (2019).

${ }^{374} \mathrm{X}$. Zheng and L. Cheng, "Performance of delta-coupled-cluster methods for calculations of core-ionization energies of first-row elements," J. Chem. Theory Comput. 15, 4945-4955 (2019).

${ }^{375}$ D. A. Matthews, "EOM-CC methods with approximate triple excitations for NEXAFS and XPS," (2020), arXiv:2001.09218 [physics.chem-ph].

${ }^{376}$ J. Lee, D. W. Small, and M. Head-Gordon, "Excited states via coupled cluster theory without equation-of-motion methods: Seeking higher roots with application to doubly excited states 
and double core hole states," J. Chem. Phys. 151, 214103 (2019).

${ }^{377}$ N. A. Besley, A. T. B. Gilbert, and P. M. W. Gill, "Self-consistent-field calculations of core excited states," J. Chem. Phys. 130, 124308 (2009).

${ }^{378}$ R. N. Tolchenov, O. Naumenko, N. F. Zobov, S. V. Shirin, O. L. Polyansky, J. Tennyson, M. Carleer, P.-F. Coheur, S. Fally, A. Jenouvrier, and A. C. Vandaele, "Water vapour line assignments in the 9250-26000cm-1 frequency range," J. Mol. Spectrosc. 233, 68-76 (2005).

${ }^{379}$ J. Tennyson, N. F. Zobov, R. Williamson, O. L. Polyansky, and P. F. Bernath, "Experimental energy levels of the water molecule," J. Phys. Chem. Ref. Data 30, 735-831 (2001).

${ }^{380}$ D. A. Clabo, W. D. Allen, R. B. Remington, Y. Yamaguchi, and H. F. Schaefer III, “A systematic study of molecular vibrational anharmonicity and vibration-rotation interaction by self-consistent-field higher-derivative methods. Asymmetric top molecules," Chem. Phys. 123, 187-239 (1988).

${ }^{381}$ A. Willetts, N. C. Handy, W. H. Green, and D. Jayatilaka, "Anharmonic corrections to vibrational transition intensities," J. Phys. Chem. 94, 5608-5616 (1990).

${ }^{382}$ T. A. Ruden, P. R. Taylor, and T. Helgaker, “Automated calculation of fundamental frequencies: Application to $\mathrm{AlH}_{3}$ using the coupled-cluster singles-and-doubles with perturbative triples method,” J. Chem. Phys. 119, 1951-1960 (2003).

${ }^{383} \mathrm{~V}$. Barone, "Anharmonic vibrational properties by a fully automated second-order perturbative approach,” J. Chem. Phys. 122, 014108 (2004).

${ }^{384}$ J. Vázquez and J. F. Stanton, "Simple(r) algebraic equation for transition moments of fundamental transitions in vibrational second-order perturbation theory," Mol. Phys. 104, 377-388 (2006).

${ }^{385}$ A. Miani, E. Cané, P. Palmieri, A. Trombetti, and N. C. Handy, "Experimental and theoretical anharmonicity for benzene using density functional theory," J. Chem. Phys. 112, 248-259 (1999).

${ }^{386}$ R. Burcl, N. C. Handy, and S. Carter, "Vibrational spectra of furan, pyrrole, and thiophene from a density functional theory anharmonic force field," Spectrochim. Acta, Part A 59, 1881-1893 (2003).

${ }^{387}$ V. Barone, G. Festa, A. Grandi, N. Rega, and N. Sanna, “Accurate vibrational spectra of large molecules by density functional computations beyond the harmonic approximation: The case of uracil and 2-thiouracil," Chem. Phys. Lett. 388, 279-283 (2004).

${ }^{388}$ H. G. Kjaergaard, A. L. Garden, G. M. Chaban, R. B. Gerber, D. A. Matthews, and J. F. Stanton, 
"Calculation of vibrational transition frequencies and intensities in water dimer: Comparison of different vibrational approaches," J. Phys. Chem. A 112, 4324-4335 (2008).

${ }^{389}$ J. F. Stanton, B. A. Flowers, D. A. Matthews, A. F. Ware, and G. B. Ellison, "Gas-phase infrared spectrum of methyl nitrate," J. Mol. Spectrosc. 251, 384-393 (2008).

${ }^{390}$ W. J. Morgan, D. A. Matthews, M. Ringholm, J. Agarwal, J. Z. Gong, K. Ruud, W. D. Allen, J. F. Stanton, and H. F. Schaefer III, "Geometric energy derivatives at the complete basis set limit: Application to the equilibrium structure and molecular force field of formaldehyde," J. Chem. Theory Comput. 14, 1333-1350 (2018).

${ }^{391}$ E. Fermi, “Über den Ramaneffekt des Kohlendioxyds,” Z. Phys. 71, 250-259 (1931).

${ }^{392}$ B. T. Darling and D. M. Dennison, “The water vapor molecule," Phys. Rev. 57, 128-139 (1940).

${ }^{393}$ J. H. Van Vleck, "On sigma-type doubling and electron spin in the spectra of diatomic molecules," Phys. Rev. 33, 467-506 (1929).

${ }^{394}$ A. B. McCoy and E. L. Sibert, "Canonical van Vleck perturbation theory and its application to studies of higly vibrationally excited states of polyatomic molecules," in Dynamics of Molecules and Chemical Reactions, edited by R. E. Wyatt and J. Z. Zhang (CRC Press, 1996) pp. 151-184.

${ }^{395}$ K. K. Lehmann, "Beyond the $\mathrm{x}-\mathrm{K}$ relations: Calculations of 1-1 and 2-2 resonance constants with application to HCN and DCN," Mol. Phys. 66, 1129-1137 (1989).

${ }^{396} \mathrm{~V}$. Hanninen and L. Halonen, "Calculation of spectroscopic parameters and vibrational overtones of methanol," Mol. Phys. 101, 2907-2916 (2003).

${ }^{397}$ J. Almlöf and P. R. Taylor, "General contraction of Gaussian basis sets. I. Atomic natural orbitals for first- and second-row atoms," J. Chem. Phys. 86, 4070-4077 (1987).

${ }^{398}$ J. Z. Gong, D. A. Matthews, P. B. Changala, and J. F. Stanton, "Fourth-order vibrational perturbation theory with the Watson Hamiltonian: Report of working equations and preliminary results," J. Chem. Phys. 149, 114102 (2018).

${ }^{399}$ J. Almlöf, K. Fægri, Jr., and K. Korsell, "Principles for a direct SCF approach to LCAO-MO ab-initio calculations,” J. Comput. Chem. 3, 385-399 (1982).

${ }^{400}$ M. Häser and R. Ahlrichs, "Improvements on the direct SCF method," J. Comput. Chem. 10, 104-111 (1989).

${ }^{401}$ M. Schütz, R. Lindh, and H.-J. Werner, "Integral-direct electron correlation methods," Mol. Phys. 96, 719-733 (1999).

${ }^{402}$ V. Dyczmons, "No $N^{4}$-dependence in the calculation of large molecules," Theor. Chem. Acc. 28, 307-310 (1973). 
${ }^{403}$ J. L. Whitten, “Coulombic potential energy integrals and approximations,” J. Chem. Phys. 58, 4496-64501 (1973).

${ }^{404}$ B. I. Dunlap, J. W. D. Connolly, and J. R. Sabin, “On some approximations in applications of $\mathrm{X}_{\alpha}$ theory," J. Chem. Phys. 71, 3396-3402 (1979).

${ }^{405}$ O. Vahtras, J. Almlöf, and M. W. Feyereisen, "Integral approximations for LCAO-SCF calculations," Chem. Phys. Lett. 213, 514-518 (1993).

${ }^{406}$ K. Eichkorn, O. Treutler, H. Öhm, M. Häser, and R. Ahlrichs, "Auxiliary basis sets to approximate Coulomb potentials," Chem. Phys. Lett. 240, 283-290 (1995).

${ }^{407}$ F. Weigend and M. Häser, "RI-MP2: First derivatives and global consistency," Theor. Chem. Acc. 97, 331-340 (1997).

${ }^{408}$ N. H. F. Beebe and J. Linderberg, "Simplifications in the generation and transformation of two-electron integrals in molecular calculations," Int. J. Quantum Chem. 12, 683-705 (1977).

${ }^{409}$ H. Koch, A. Sánchez de Merás, and T. B. Pedersen, "Reduced scaling in electronic structure calculations using Cholesky decompositions," J. Chem. Phys. 118, 9481-9484 (2003).

${ }^{410}$ F. Aquilante, R. Lindh, and T. B. Pedersen, "Unbiased auxiliary basis sets for accurate twoelectron integral approximations," J. Chem. Phys. 127, 114107 (2007).

${ }^{411}$ L. Boman, H. Koch, and A. Sánchez de Merás, "Method specific Cholesky decomposition: Coulomb and exchange energies," J. Chem. Phys. 129, 134107 (2008).

${ }^{412}$ F. Aquilante, T. B. Pedersen, and R. Lindh, "Density fitting with auxiliary basis sets from Cholesky decompositions,” Theor. Chem. Acc. 124, 1-10 (2009).

${ }^{413}$ F. Aquilante, L. Gagliardi, T. B. Pedersen, and R. Lindh, "Atomic Cholesky decompositions: A route to unbiased auxiliary basis sets for density fitting approximation with tunable accuracy and efficiency,” J. Chem. Phys. 130, 154107 (2009).

${ }^{414}$ E. Epifanovsky, D. Zuev, X. Feng, K. Khistyaev, Y. Shao, and A. I. Krylov, "General implementation of the resolution-of-the-identity and Cholesky representations of electron repulsion integrals within coupled-cluster and equation-of-motion methods: Theory and benchmarks," J. Chem. Phys. 139, 134105 (2013).

${ }^{415}$ S. D. Folkestad, E. F. Kjønstad, and H. Koch, “An efficient algorithm for Cholesky decomposition of electron repulsion integrals," J. Chem. Phys. 150, 194112 (2019).

${ }^{416}$ K. Eichkorn, F. Weigend, O. Treutler, and R. Ahlrichs, "Auxiliary basis sets for main row atoms and transition metals and their use to approximate Coulomb potentials," Theor. Chem. Acc. 97, 119-124 (1995). 
${ }^{417}$ F. Weigend, M. Häser, H. Patzelt, and R. Ahlrichs, "RI-MP2: Optimized auxiliary basis sets and demonstration of efficiency," Chem. Phys. Lett. 294, 143-152 (1998).

${ }^{418} \mathrm{~F}$. Weigend, A. Köhn, and C. Hättig, "Efficient use of the correlation consistent basis sets in resolution of the identity MP2 calculations,” J. Chem. Phys. 116, 3175-3183 (2002).

${ }^{419}$ F. Weigend, “Accurate Coulomb-fitting basis sets for H to Rn," Phys. Chem. Chem. Phys. 8, 1057-1065 (2006).

${ }^{420}$ J. Boström, V. Veryazov, F. Aquilante, T. B. Pedersen, and R. Lindh, "Analytical gradients of the second-order Møller-Plesset energy using Cholesky decompositions,” Int. J. Quantum Chem. 114, 321-327 (2014).

${ }^{421}$ M. Delcey, T. B. Pedersen, F. Aquilante, and R. Lindh, "Analytical gradients of the stateaverage complete active space self-consistent field method with density fitting," J. Chem. Phys. 143, 044110 (2015).

${ }^{422}$ X. Feng, E. Epifanovsky, J. Gauss, and A. I. Krylov, "Implementation of analytic gradients for CCSD and EOM-CCSD using Cholesky decomposition of the electron-repulsion integrals and their derivatives: Theory and benchmarks," J. Chem. Phys. 151, 014110 (2019).

${ }^{423} \mathrm{~F}$. Aquilante and T. B. Pedersen, "Quartic scaling evaluation of canonical scaled opposite spin second-order Møller-Plesset correlation energy using Cholesky decompositions," Chem. Phys. Lett. 449, 354-357 (2007).

${ }^{424}$ J. Yousung, S. Yihan, and M. Head-Gordon, "Fast evaluation of scaled opposite spin secondorder Møller-Plesset correlation energies using auxiliary basis expansions and exploiting sparsity," J. Comput. Chem. 28, 1953-1964 (2007).

${ }^{425}$ M. Hilgenberg, "Die Verwendung der Cholesky-Zerlegung in der Coupled-Cluster-Theorie (in German), Johannes Gutenberg-Universität Mainz, diploma thesis (2017).

${ }^{426}$ E. G. Hohenstein, R. M. Parrish, and T. J. Martínez, “Tensor hypercontraction density fitting. I. Quartic scaling second- and third-order Møller-Plesset perturbation theory," J. Chem. Phys. 137, 044103 (2012).

${ }^{427}$ R. M. Parrish, E. G. Hohenstein, T. J. Martínez, and C. D. Sherrill, "Tensor hypercontraction. II. Least-squares renormalization,” J. Chem. Phys. 137, 224106 (2012).

${ }^{428}$ D. A. Matthews, "Improved Grid Optimization and Fitting in Least Squares Tensor Hypercontraction,” J. Chem. Theory Comput. (2020), 10.1021/acs.jctc.9b01205.

${ }^{429}$ S. I. L. Kokkila Schumacher, E. G. Hohenstein, R. M. Parrish, L.-P. Wang, and T. J. Martínez, “Tensor Hypercontraction Second-Order Møller-Plesset Perturbation Theory: Grid Optimiza- 
tion and Reaction Energies," J. Chem. Theory Comput. 11, 3042-3052 (2015).

${ }^{430}$ R. M. Parrish, E. G. Hohenstein, T. J. Martínez, and C. D. Sherrill, "Discrete variable representation in electronic structure theory: Quadrature grids for least-squares tensor hypercontraction,” J. Chem. Phys. 138, 194107 (2013).

${ }^{431}$ J. Lee, L. Lin, and M. Head-Gordon, "Systematically Improvable Tensor Hypercontraction: Interpolative Separable Density-Fitting for Molecules Applied to Exact Exchange, Secondand Third-Order Møller-Plesset Perturbation Theory," J. Chem. Theory Comput. 16, 243-263 (2020).

${ }^{432}$ E. G. Hohenstein, R. M. Parrish, C. D. Sherrill, and T. J. Martínez, "Communication: Tensor hypercontraction. III. Least-squares tensor hypercontraction for the determination of correlated wavefunctions,” J. Chem. Phys. 137, 221101 (2012).

${ }^{433}$ S. Stopkowicz, "Perspective: Coupled cluster theory for atoms and molecules in strong magnetic fields,” Int. J. Quantum Chem. 18, e25391 (2017).

${ }^{434}$ K. K. Lange, E. I. Tellgren, M. R. Hoffmann, and T. Helgaker, "A paramagnetic bonding mechanism for diatomics in strong magnetic fields," Science 337, 327-331 (2012).

${ }^{435}$ S. Jordan, P. Schmelcher, W. Becken, and W. Schweizer, "Evidence for helium in the magnetic white dwarf GD 229," Astron. Astrophys. 336, L33-L36 (1988).

${ }^{436}$ S. Stopkowicz, J. Gauss, K. K. Lange, E. I. Tellgren, and T. Helgaker, "Coupled-cluster theory for atoms and molecules in strong magnetic fields,” J. Chem. Phys. 143, 074110 (2015).

${ }^{437}$ F. Hampe and S. Stopkowicz, "Equation-of-motion coupled-cluster methods for atoms and molecules in strong magnetic fields," J. Chem. Phys. 146, 154105 (2017).

${ }^{438}$ F. Hampe and S. Stopkowicz, "Transition-dipole moments for electronic excitations in strong magnetic fields using equation-of-motion and linear response coupled-cluster theory," J. Chem. Theory Comput. 15, 4036-4043 (2019).

${ }^{439}$ L. E. McMurchie and E. R. Davidson, "One- and two-electron integrals over Cartesian Gaussian functions," J. Comput. Phys. 26, 218-231 (1978).

${ }^{440}$ E. I. Tellgren, A. Soncini, and T. Helgaker, "Nonperturbative ab initio calculations in strong magnetic fields using London orbitals," J. Chem. Phys. 129, 154114 (2008).

${ }^{441}$ F. Hampe, S. Stopkowicz, N. Gross, and M.-P. Kitsaras, "QCUMBRE, quantum chemical utility enabling magnetic-field dependent investigations benefitting from rigorous electroncorrelation treatment,". 


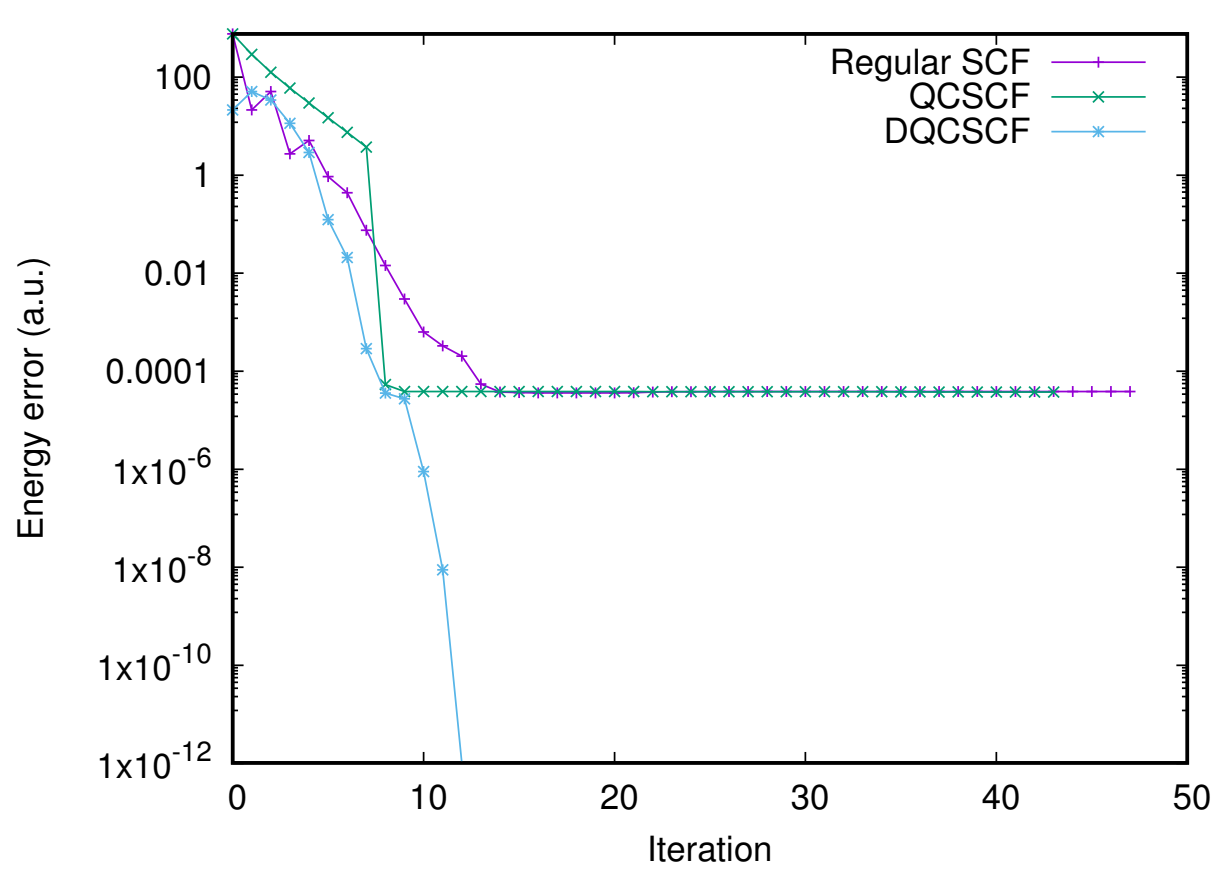



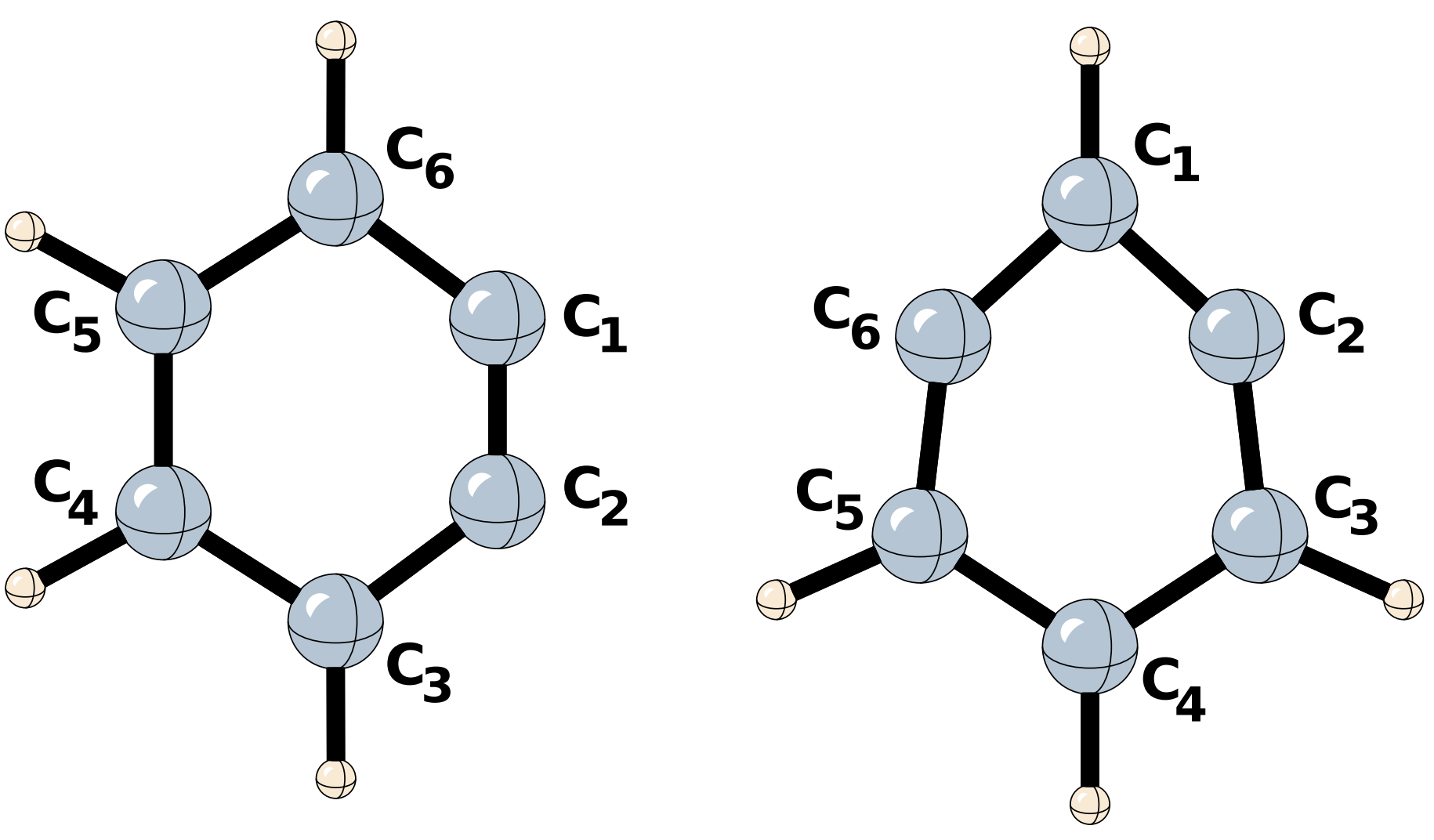

o-benzyne

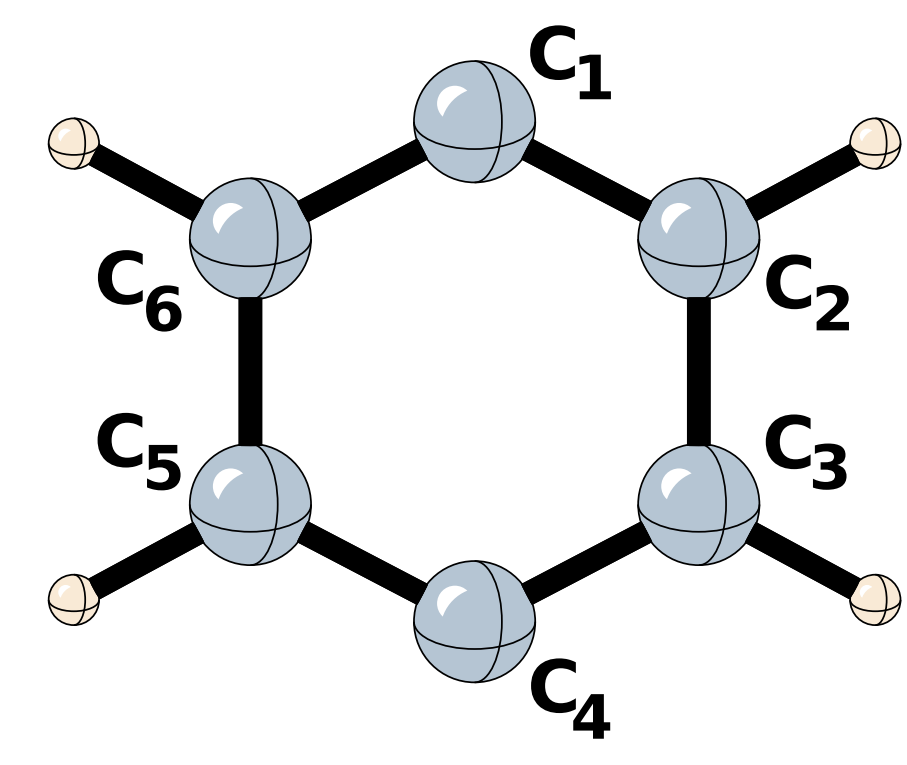

p-benzyne 


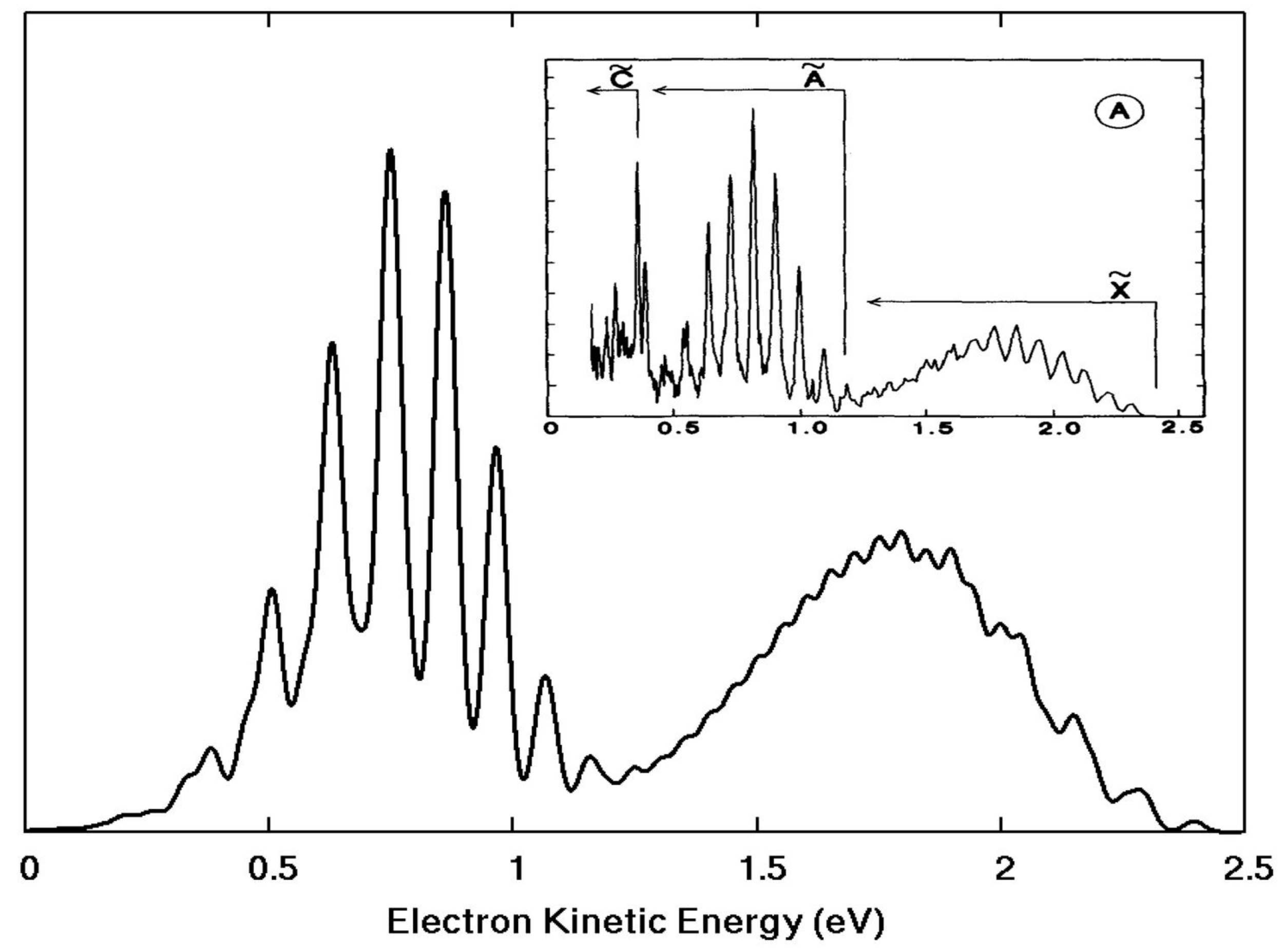




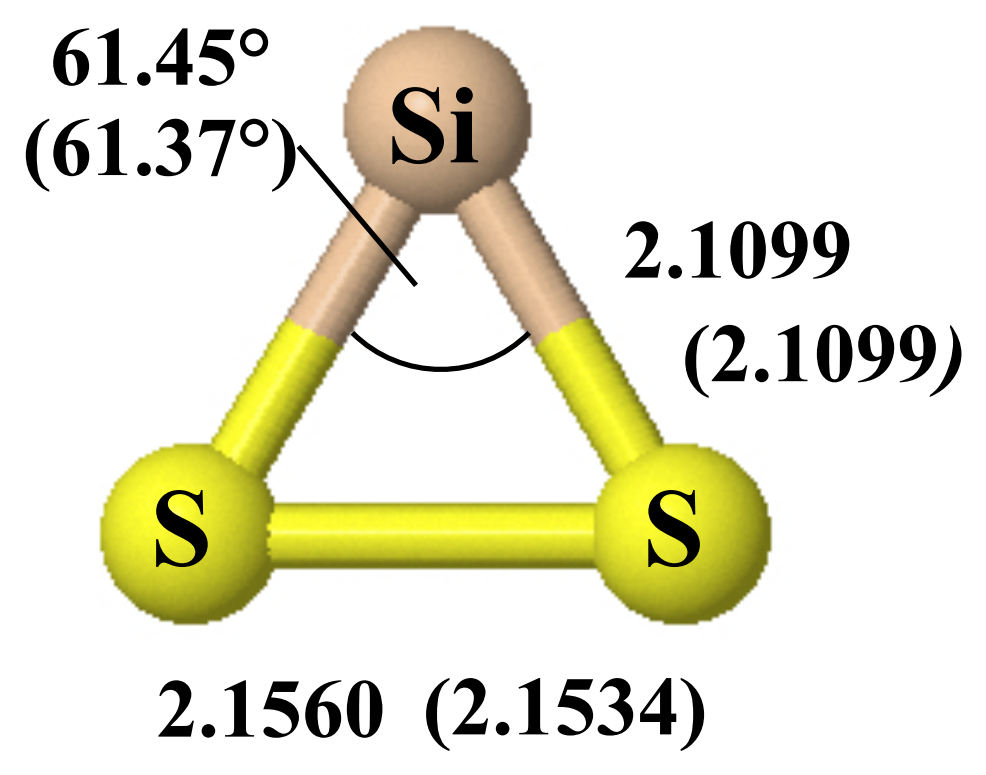




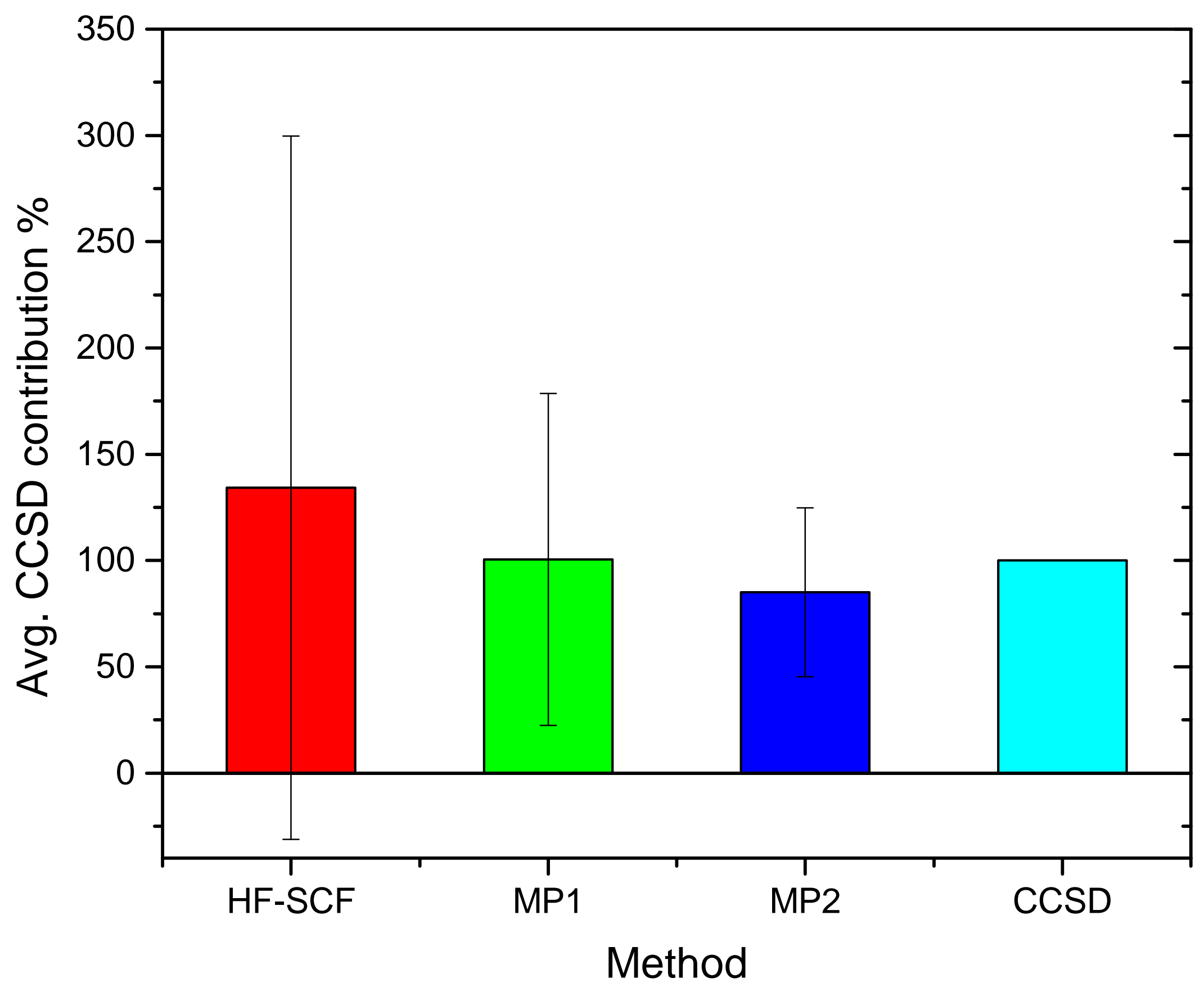

